\title{
Conjugates of Betulin Derivatives with AZT as Potent Anti-HIV
}

\section{Agents}

\author{
Juan Xionga , Yoshiki Kashiwadaa, ${ }^{\text {, }}$, Chin-Ho Chen ${ }^{b}$, Keduo Qianc, Susan L. Morris-

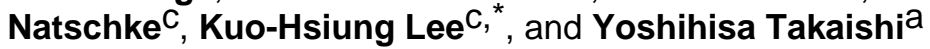 \\ aGraduate School of Pharmaceutical Sciences, University of Tokushima, Tokushima 770-8505, \\ Japan \\ bMedical Center, Duke University, Durham, NC 27710, USA \\ cNatural Products Research Laboratories, Eshelman School of Pharmacy, University of North \\ Carolina, Chapel Hill, NC 27599-7568, USA
}

\begin{abstract}
Fourteen novel conjugates of 3,28-di-O-acylbetulins with AZT were prepared as anti-HIV agents, based on our previously reported potent anti-HIV triterpene leads, including 3-O-acyl and 3,28-di$O$-acylbetulins. Nine of the conjugates $(\mathbf{4 9 - 5 3 , 5 5 , 5 6 , 5 9 , 6 0})$ exhibited potent anti-HIV activity at the submicromolar level, with $\mathrm{EC}_{50}$ values ranging from 0.040 to $0.098 \mu \mathrm{M}$ in $\mathrm{HIV}-1_{\mathrm{NL} 4-3}$ infected MT-4 cells. These compounds were equipotent or more potent than 3-O-(3',3'dimethylsuccinyl)betulinic acid (2), which is currently in Phase IIb anti-AIDS clinical trial.
\end{abstract}

\section{Keywords}

HIV-1; Betulin; AZT; Conjugate

\section{Introduction}

Betulinic acid (1), a lupane-type triterpene, is widely distributed throughout the plant kingdom. Various biological activities ${ }^{1-5}$ have been reported for $\mathbf{1}$, including anti-HIV, anticancer, and anti-inflammatory properties. Our previous modification study on $\mathbf{1}$ led to the discovery of 3-O-(3',3'-dimethylsuccinyl)betulinic acid (2, bevirimat, now also known as MPC-4326). Compound 2 exhibits potent anti-HIV activity and is currently in phase IIb clinical trials in the US. $6^{-9}$ It is a first-in-class drug candidate as a viral maturation inhibitor, which disrupts the processing of capsid precursor p25 (CA-SP1) to mature capsid protein p24 (CA), resulting in generation of non-infectious HIV-1 virions. ${ }^{10}$

In subsequent research, various derivatives of betulin (3a) and dihydrobetulin (3b) were investigated based on the structural similarity of $\mathbf{1}$ and $\mathbf{3 a}, \mathbf{3 b} \mathbf{b}^{1{ }^{1-14}}$ Several analogs, including 3-O-glutaryldihydrobetulin (4) ${ }^{14}$ 3,28-di- $O$-(3',3'-dimethylglutaryl)betulin (5),

\footnotetext{
(C) 2010 Elsevier Ltd. All rights reserved.

* Corresponding authors. Tel/Fax: +81-88-633-7276. kasiwada@ph.tokukshima-u.ac.jp (Y. Kashiwada)., Tel: +1 919 962 0066; Fax: +19199663893. khlee@unc.edu (K-H Lee).

Publisher's Disclaimer: This is a PDF file of an unedited manuscript that has been accepted for publication. As a service to our customers we are providing this early version of the manuscript. The manuscript will undergo copyediting, typesetting, and review of the resulting proof before it is published in its final citable form. Please note that during the production process errors may be discovered which could affect the content, and all legal disclaimers that apply to the journal pertain.
} 
and 3-O-(3',3'-dimethylsuccinyl)-28-O-(2",2"-dimethylsuccinyl)betulin (6) ${ }^{12,13}$ exhibited comparable or greater anti-HIV activity compared with the structurally related 2(Figure 1). ${ }^{15}$

In a continuing study of potent anti-HIV agents based on the betulin scaffold, we wanted to explore the conjugation of our unique HIV-1 maturation inhibitors with other classes of antiHIV agents, because the strategy of multi-target therapeutics could be more efficacious and less prone to resistance than monotherapies. ${ }^{16,} 17$ Although it is more challenging to create multi-target therapeutics by building multi-target actions into one single chemical entity rather than mixing monotherapies, 16,18 the multi-targeted single agent could greatly simplify treatment regimens and reduce the risk of drug-drug interactions. 16 Herein, we report our preliminary research on conjugation of 3'-azido-3'-deoxythymidine (AZT), ${ }^{19}$ the first clinically approved nucleoside reverse transcriptase inhibitor (NRTI), with betulin derivatives. The hybrid conjugates were formed through a linker with ester bonds, which are easy to hydrolyze and subsequently release the parent compounds, betulin derivative and AZT, to exert their functions.

\section{Design}

Initially, we considered where to link AZT to the triterpene skeleton. 3,28-Di- $O$-acylbetulin derivatives contain two free carboxylic acid groups, which appear to be ideal fragments for constructing conjugates. Previous studies suggested that the free terminal carboxylic acid in the 3-O-acyl group is essential for anti-maturation activity; thus, the other-COOH group in the 28-O-acyl moiety was considered to be the appropriate conjugating site (Fig. 2.).

Secondly, we considered the identity of the 3-O-acyl group $\left(>\mathrm{R}_{1}\right)$. Our prior modification studies on 1, 3a and $\mathbf{3 b}$ showed that the esterification of the 3-OH with $33^{\prime}, 3^{\prime}-$ dimethylglutaryl or 3',3'-dimethylsuccinyl fragments produced significantly active derivatives (e.g., 5 and 6). ${ }^{6,12,14,20-22}$ However, the 3a-derivatives bearing a 3-O-glutaryl group exhibited only weak or moderate anti-HIV activity. ${ }^{14}$ In contrast, introduction of a glutaryl group onto the $3-\mathrm{OH}$ of $\mathbf{3 b}$ led to a dramatic increase of anti-HIV potency as seen in compound $\mathbf{4}$. Therefore, in this study, triterpenes with the lup-20(29)-ene scaffold of 3a were acylated with 3,3-dimethylglutaryl (54) and 3,3-dimethylsuccinyl (49-53) moieties, while those with the lupine scaffold of $\mathbf{3 b}$ were also acylated with a glutaryl group (58-61), as well as the two preceding moieties (55-57 and 62).

Thirdly, we considered the identity of the $28-O$-acyl group $\left(\mathrm{R}_{2}\right)$. Although previous biological data for betulin derivatives suggested that the group at this position might be not essential for the anti-HIV activity of this compound type, it could still have some influence on potency. ${ }^{12}$ In addition, here the 28 - $O$-acyl group is also being used as a linker in the conjugates. Therefore, the identity of the linking ester group might play a vital part in the anti-HIV activity of hybrid conjugates. Among the 3,28-di- $O$-acylbetulin derivatives previously studied, compounds $\mathbf{5}$ and $\mathbf{6}$ exhibited the greatest potency, and thus the 3,3dimethylglutaryl and 2,2-dimethylsuccinyl groups found in these two compounds were used for constructing the triterpene-AZT conjugates. Succinyl, glutaryl, and 3,3-dimethylsuccinyl esters were also investigated as the $28-O$-acyl moiety in the conjugate compounds.

The preparation of fourteen new conjugates of 3,28-di-O-acylbetulins with AZT and evaluation of anti-HIV activity are described in this paper. Table 1 lists the structural identities of the 3- and 28-O-acyl side chains, and the degree of saturation of the $\mathrm{C} 20-\mathrm{C} 29$ bond for all intermediates (21-48) and final conjugates (49-62). 


\section{Chemistry}

The synthetic route to conjugated products (49-62) is outlined in Scheme 1 and Scheme 2. The key intermediate 3-O-acylbetulins (11-13) were successfully prepared by the methods described previously ${ }^{14}$ as shown in Scheme 1. Protection of the 28-OH group with triphenylmethyl (or trityl) chloride yielded betulin 28-O-trityl ether (7), which was further treated with an appropriate dicarboxylic acid or anhydride in anhydrous pyridine in the presence of 4-dimethylaminopyridine (DMAP) to furnish the corresponding 3-O-acyl-28- $O$ tritylbetulins (8-10). As was found in previous studies, the reaction of 7 with 2,2dimethylsuccinic acid gave a mixture of 3-O-2',2'-dimethylsuccinyl- and -3',3'dimethylsuccinylbetulin derivatives, in which the latter isomer (8) was the major product. The isomers were separated by silica gel chromatography, and their structures were assigned by $2 \mathrm{D}-\mathrm{NMR}$ analysis (HMBC). For $\mathbf{8}$ and $\mathbf{9}$, the protecting trityl group was subsequently cleaved with pyridium $p$-toluenesulfonate (PPTS) in $\mathrm{CH}_{2} \mathrm{Cl}_{2}$-EtOH to yield 3-Oacylbetulins (11 and 12, respectively). An ethyl ester side-product was obtained predominately by removing the 28-O-trityl group from 3-O-glutaryl-28- $O$-tritylbetulin (10) with PPTS; however, the desired product (13) was obtained exclusively by refluxing 10 with Lewis acid $\mathrm{FeCl}_{3}$ in $95 \%$ THF.

Hydrogenation of 3-O-acylbetulins (11-13) with $\mathrm{H}_{2} / \mathrm{Pd}-\mathrm{C}$ in EtOAc-EtOH afforded the 3-Oacyldihydrobetulins $(\mathbf{1 4}, \mathbf{1 5}$, and $\mathbf{4}$, respectively) almost quantitatively. Before a second acyl moiety was introduced at the $\mathrm{C}-28$ position, the free terminal carboxylic acid in the 3-O-acyl group was first protected as the diphenyldiazomethyl ester. Treatment of 3-O-acylbetulins $(11,12)$ and -dihydrobetulins $(14,15,4)$ with freshly prepared diphenyldiazomethane in $\mathrm{MeOH}$ produced the protected derivatives (16-20) in quantitative yield.

The 3,28-di- $O$-acylbetulins (21-34) were readily prepared by heating the protected 3-Oacylbetulin derivatives with an appropriate dicarboxylic acid or anhydride overnight in the presence of DMAP and dry pyridine (Scheme 2). The two isomeric products obtained when a dimethylsuccinyl group was introduced at C-28 of 16, 18, and 20 were successfully separated by preparative HPLC, yielding corresponding pure derivatives. The orientation of the dimethylsuccinyl group attached to the C-28 hydroxy group of $\mathbf{2 2 / 2 3 , 2 7 / 2 8}$ and $\mathbf{3 0 / 3 1}$ was established by 2D NMR analyses (HSQC and HMBC). The 28-O-(3",3"dimethylsuccinyl)betulin derivative was the major product in each case. The electrondonating effect of the dimethyl groups on the adjacent carboxyl group would cause decreased electrophilicity, resulting in different yields of the two isomers in acylation reactions either at C-3 or C-28.

Conjugation of the 3,28-di-O-acylbetulin (21-26) and-dihydrobetulin derivatives (27-34) with AZT in the presence of dicyclohexylcarbodiimide (DCC) and DMAP in $\mathrm{CH}_{2} \mathrm{Cl}_{2}$ gave the corresponding conjugates (35-48). However, the corresponding anhydride was also obtained in each case, resulting in only a moderate yield of the desired conjugate. Finally, the terminal protecting group on the 3-O-acyl moiety was removed with $80 \%$ acetic acid to afford the target conjugates (49-62).

\section{Results and Discussion}

The anti-HIV-1 replication activities of the newly synthesized conjugates (49-62) were evaluated in HIV-1 $1_{\text {NL4-3 }}$ infected MT- 4 cells in parallel with AZT. The bioassay results are summarized in Table 2.

In the MT-4 screening system, nine conjugates $(\mathbf{4 9 - 5 3 , 5 5 , 5 6 , 5 9 , 6 0})$ exhibited potent antiHIV activity at a submicromolar level ( $\mathrm{EC}_{50}$ values ranging from $0.040 \mu \mathrm{M}$ to $0.098 \mu \mathrm{M}$ ), and thus, were equipotent or more potent than 2 . Compounds 51 and $\mathbf{4 9}$, which have a 3-O- 
$\left(3^{\prime}, 3^{\prime}\right.$-dimethylsuccinyl) moiety together with a $28-O-\left(2^{\prime \prime}, 2^{\prime \prime}\right.$-dimethylsuccinyl) or 28-Osuccinyl group, respectively, linked to AZT, exhibited the highest potency, with $\mathrm{EC}_{50}$ values of 0.040 and $0.045 \mu \mathrm{M}$, respectively. Interestingly, all three conjugates $(\mathbf{5 1 , 5 6}$ and $\mathbf{5 9})$ with a 2,2-dimethylsuccinyl linking group at C-28 generally showed higher potency than their corresponding congeners with the same 3-O-acyl group, but a different 28-O-acyl group (51 compared with 50, 52 and $53 ; 56$ compared with 55 and $57 ; 59$ compared with 58,60 , and 61). Of particular note, the conjugates $(\mathbf{5 0}, \mathbf{5 5}$, and $\mathbf{5 8}$ ) with a 3,3-dimethylsuccinyl moiety as the linking unit at C-28 showed decreased activity in HIV-1 infected MT-4 cells relative to those with a 2,2-dimethylsuccinyl group $(\mathbf{5 1}, \mathbf{5 6}$, and 59). These results confirmed our previous finding that a 2,2-dimethylsuccinyl is superior to a 3,3-dimethylsuccinyl side chain at the C-28 position of anti-HIV triterpene analogs. For example, compound $\mathbf{6}$ was reported to be 230-fold more potent against HIV replication in $\mathrm{H} 9$ lymphocytes assay than its isomer with a 3,3-dimethylsuccinyl side chain at C-28. ${ }^{13}$

Among conjugates containing glutaryl or dimethyglutaryl esters, 59 and 60 [3-O-glutaryl, $28-O$-(2",2"-dimethylsuccinyl), $\mathrm{EC}_{50} 0.073 \mu \mathrm{M}$ and 3,28-di- $O$-glutaryl, $\left.\mathrm{EC}_{50} 0.093 \mu \mathrm{M}\right]$ were equipotent with $\mathbf{2}$. However, 54 and $\mathbf{6 2}$ [3,28-di-O-(3',3'-dimethylglutaryl)] showed only moderate activity, and 61 [3-O-glutaryl, 28-O-(3",3"-glutaryl)] was the weakest compound.

Comparison of the anti-HIV potency of dihydrobetulin conjugates 55-57 with that of betulin conjugates 50, 51, and $\mathbf{5 3}$ suggested that hydrogenation of the isopropylidene group at C-19 did not help to increase the anti-HIV potency. Finally, comparison of data for 49/63 and 52/64 in Table 3 showed that hybrid conjugate compounds were four- and five-fold more active, respectively, than the corresponding compounds not linked to AZT.

In conclusion, fourteen novel conjugates of 3,28-di- $O$-acyl-betulins with AZT were prepared as anti-HIV-1 agents. In MT-4 cells, nine of the conjugates demonstrated potent anti-HIV activity at a submicromolar level with $\mathrm{EC}_{50}$ values ranging from $0.040 \mu \mathrm{M}$ to $0.098 \mu \mathrm{M}$, and thus, were equipotent or more potent than 2. A 2,2-dimethylsuccinyl group at the C-28 position served as the best linker between the triterpene scaffold and AZT in this study.

\section{Experimental Section}

\subsection{General Experimental Procedures}

Optical rotations were measured with a JASCO P-2200 digital polarimeter. NMR spectra (400 MHz for ${ }^{1} \mathrm{H}, 100 \mathrm{MHz}$ for ${ }^{13} \mathrm{C}$, using TMS as internal standard) were recorded on a Bruker AVANCE 400 spectrometer. HRESIMS was obtained on a Waters LCT Premier. Column chromatography was performed with silica gel $60 \mathrm{~N}$ (63-210 nm, Merck); Preparative HPLC was carried out on Mightysil RP-18 GP column $(250 \times 20 \mathrm{~mm}$ i.d., Kanto Chemical Co., Inc.) or Cosmosil column (Cholester, $250 \times 20 \mathrm{~mm}$ i.d.; $\pi$ NAP, $250 \times 20 \mathrm{~mm}$ i.d.; Nacalai Tesque). Unless otherwise indicated, all reagents were purchased from commercial suppliers and used without further purification.

\subsection{Chemical preparation}

4.2.1 28-O-Tritylbetulin (7)-To a solution of 3a (1.02 g, $2.30 \mathrm{mmol})$ in DMF (15 mL) were added dimethylaminopyridine (DMAP, $0.33 \mathrm{~g}, 2.70 \mathrm{mmol}$ ) and triphenylmethyl chloride $(1.26 \mathrm{~g}, 4.52 \mathrm{mmol})$. After refluxing for $6 \mathrm{~h}$, the reaction mixture was poured into ice water, and extracted with $\mathrm{CHCl}_{3}$. The organic layer was washed with $2 \mathrm{~N} \mathrm{HCl}$ solution and brine, dried over $\mathrm{Na}_{2} \mathrm{SO}_{4}$ and concentrated. The obtained residue was chromatographed over silica gel column (hexane/EtOAc $=10: 1)$ to afford 7 as a white solid $(1.16 \mathrm{~g}, 73.5 \%$ yield). $[\alpha]_{\mathrm{D}}^{25}-2.1^{\circ}\left(c 1.49, \mathrm{CHCl}_{3}\right) .{ }^{1} \mathrm{H} \mathrm{NMR}\left(\mathrm{CDCl}_{3}, 400 \mathrm{MHz}\right) \delta 0.54\left(3 \mathrm{H}, \mathrm{s}, \mathrm{CH}_{3}-26\right)$, 
0.77 (3H, s, $\left.\mathrm{CH}_{3}-24\right), 0.78$ (3H, s, $\left.\mathrm{CH}_{3}-25\right), 0.91$ (3H, s, $\left.\mathrm{CH}_{3}-27\right), 0.97$ (3H, s, $\mathrm{CH}_{3}-23$ ), $1.65\left(3 \mathrm{H}, \mathrm{s}, \mathrm{CH}_{3}-30\right), 2.15-2.24(3 \mathrm{H}, \mathrm{m}, \mathrm{H}-22, \mathrm{H}-16$ and $\mathrm{H}-19), 2.92(1 \mathrm{H}, \mathrm{d}, J=8.8 \mathrm{~Hz}$, $\mathrm{H}-28$ ), 3.15-3.19 (2H, m, H-28 and H-3), 4.53, 4.59 (each $1 \mathrm{H}$, br s, $\left.\mathrm{H}_{2}-29\right), 7.23$ (3H, t, $J=$ $7.2 \mathrm{~Hz}$, trityl H-4'), $7.31(6 \mathrm{H}, \mathrm{t}, J=7.6,7.2 \mathrm{~Hz}$, trityl H-3',5'), $7.49(6 \mathrm{H}, \mathrm{d}, J=7.6 \mathrm{~Hz}$, trityl $\left.\mathrm{H}-2 ', 6{ }^{\prime}\right) ;{ }^{13} \mathrm{C}$ NMR $\left(\mathrm{CDCl}_{3}, 100 \mathrm{MHz}\right) \delta 14.7$ (C-27), 15.3 (C-24), 15.9 (C-26), 16.1 (C-25), 18.3 (C-6), 19.1 (C-30), 20.7 (C-11), 25.2 (C-12), 26.9 (C-15), 27.4 (C-2), 28.0 (C-23), 29.9 (C-21), 30.2 (C-16), 34.2 (C-7), 35.2 (C-22), 37.1 (C-10), 37.3 (C-13), 38.7 (C-1), 38.8 (C-4), 40.6 (C-8), 42.5 (C-14), 47.6 (C-17), 47.7 (C-19), 48.9 (C-18), 50.3 (C-9), 55.3 (C-5), $59.6(\mathrm{C}-28), 79.0(\mathrm{C}-3), 85.9\left(\operatorname{trityl} \mathrm{C}(\mathrm{Ph})_{3}\right), 109.3(\mathrm{C}-29), 126.8$ (trityl C-4'), 127.7 (trityl C-2', 6'), 128.8 (trityl C-3', 5'), 144.5 (trityl C-1'), 150.8 (C-20). HRESIMS (positive) $\mathrm{m} / \mathrm{z}$ $707.4781[\mathrm{M}+\mathrm{Na}]^{+}$(calcd for $\mathrm{C}_{49} \mathrm{H}_{64} \mathrm{O}_{2} \mathrm{Na}, 707.4804$ ).

\subsubsection{General procedure for preparation of 3-O-acyl-28-O-tritylbetulins (8-10)}

-3-O-Acyl-28-O-tritylbetulins were prepared by refluxing a solution of 7 (1 equiv), appropriate dicarboxylic acid (4 equiv) or anhydride (4-5 equiv) and DMAP (1-2 equiv) in anhydrous pyridine (10-20 mL) overnight. After cooling to room temperature, the reaction mixture was diluted with ice-water, and extracted with $\mathrm{CHCl}_{3}$. The organic layer was washed with water, $2 \mathrm{~N} \mathrm{HCl}$ solution and brine, dried over $\mathrm{Na}_{2} \mathrm{SO}_{4}$, and concentrated. The crude product was purified by silica gel column chromatography (hexane/EtOAc $=10: 1$ ).

3-O-(3',3'-Dimethylsuccinyl)-28- $\boldsymbol{O}$-tritylbetulin (8): Yield 38.9\% (starting from $4.48 \mathrm{~g}$ of 7 and 2,2-dimethylsuccinic acid); white solid; 3-O-(2',2'-dimethylsuccinyl)-28- $O$ -

tritylbetulin was also separated as by-product $(11.1 \%$ yield $) \cdot[\alpha]_{\mathrm{D}}^{25}+2.1^{\circ}\left(c 1.25, \mathrm{CHCl}_{3}\right) .{ }^{1} \mathrm{H}$ NMR $\left(\mathrm{CDCl}_{3}, 400 \mathrm{MHz}\right) \delta 0.53\left(3 \mathrm{H}, \mathrm{s}, \mathrm{CH}_{3}-26\right), 0.79\left(3 \mathrm{H}, \mathrm{s}, \mathrm{CH}_{3}-25\right), 0.81(3 \mathrm{H}, \mathrm{s}$, $\left.\mathrm{CH}_{3}-24\right), 0.84\left(3 \mathrm{H}, \mathrm{s}, \mathrm{CH}_{3}-23\right), 0.90$ (3H, s, $\left.\mathrm{CH}_{3}-27\right), 1.30,1.31$ (each $3 \mathrm{H}, \mathrm{s}$, dimethylsuccinyl $\mathrm{CH}_{3}$ ), 1.65 (3H, s, $\left.\mathrm{CH}_{3}-30\right), 2.15-2.24$ (3H, m, H-22, H-16 and H-19), 2.57, 2.68 (each $1 \mathrm{H}, \mathrm{d}, J=15.6 \mathrm{~Hz}$, dimethylsuccinyl $\mathrm{H}_{2}-2^{\prime}$ ), 2.92, 3.15 (each $1 \mathrm{H}, \mathrm{d}, J=8.8$ $\left.\mathrm{Hz}, \mathrm{H}_{2}-28\right), 4.48(1 \mathrm{H}, \mathrm{dd}, J=5.2,11.2 \mathrm{~Hz}, \mathrm{H}-3), 4.53,4.59$ (each $1 \mathrm{H}$, br s, $\left.\mathrm{H}_{2}-29\right), 7.23$ $\left(3 \mathrm{H}, \mathrm{t}, J=7.2 \mathrm{~Hz}\right.$, trityl H-4'), $7.31\left(6 \mathrm{H}, \mathrm{t}, J=8.0,7.2 \mathrm{~Hz}\right.$, trityl H-3', $\left.5^{\prime}\right), 7.50(6 \mathrm{H}, \mathrm{d}, J=8.0$ $\mathrm{Hz}$, trityl H-2',6'); ${ }^{13} \mathrm{C}$ NMR $\left(\mathrm{CDCl}_{3}, 100 \mathrm{MHz}\right) \delta 14.7$ (C-27), 15.9 (C-26), 16.1 (C-25), 16.5 (C-24), 18.2 (C-6), 19.1 (C-30), 20.7 (C-11), 23.6 (C-2), 25.1 (C-12), 25.0, 25.6 (dimethylsuccinyl $\mathrm{CH}_{3}$ ), 26.9 (C-15), 27.9 (C-23), 29.9 (C-21), 30.1 (C-16), 34.1 (C-7), 35.2 (C-22), 37.0 (C-10), 37.2 (C-13), 37.7 (C-4), 38.3 (C-1), 40.5 (dimethylsuccinyl C-3'), 40.6 (C-8), 42.5 (C-14), 44.7 (dimethylsuccinyl C-2'), 47.6 (C-17), 47.8 (C-19), 48.9 (C-18), 50.2 (C-9), 55.3 (C-5), 59.5 (C-28), 81.6 (C-3), 85.8 (trityl C(Ph) 3 ), 109.4 (C-29), 126.8 (trityl C-4'), 127.7 (trityl C-2', 6'), 128.8 (trityl C-3', 5'), 144.5 (trityl C-1'), 150.8 (C-20), 170.9 (dimethylsuccinyl COO-), 182.8 (dimethylsuccinyl COOH). HRESIMS (positive) $\mathrm{m} / \mathrm{z}$ 835.5286 [M+Na] ${ }^{+}$(calcd for $\mathrm{C}_{55} \mathrm{H}_{72} \mathrm{O}_{5} \mathrm{Na}$, 835.5277).

3-O-(3',3'-Dimethylglutaryl)-28-O-tritylbetulin (9): Yield 86.6\% (starting from $956 \mathrm{mg}$ of 7 and 3,3-dimethylglutaric anhydride); white solid. $[\alpha]_{\mathrm{D}}^{25}+7.5^{\circ}\left(c 0.52, \mathrm{CHCl}_{3}\right) .{ }^{1} \mathrm{H}$ NMR $\left(\mathrm{CDCl}_{3}, 400 \mathrm{MHz}\right) \delta 0.55\left(3 \mathrm{H}, \mathrm{s}, \mathrm{CH}_{3}-26\right), 0.81\left(3 \mathrm{H}, \mathrm{s}, \mathrm{CH}_{3}-25\right), 0.85$ (3H, s, $\left.\mathrm{CH}_{3}-24\right), 0.86$ $\left(3 \mathrm{H}, \mathrm{s}, \mathrm{CH}_{3}-23\right), 0.91\left(3 \mathrm{H}, \mathrm{s}, \mathrm{CH}_{3}-27\right), 1.16\left(6 \mathrm{H}, \mathrm{s}\right.$, dimethylglutaryl $\left.\mathrm{CH}_{3}\right), 1.65(3 \mathrm{H}, \mathrm{s}$, $\mathrm{CH}_{3}-30$ ), 2.15-2.24 (3H, m, H-22, H-16 and H-19), 2.40, 2.47 (each 1H, d, $J=14.0 \mathrm{~Hz}$, dimethylglutaryl $\left.\mathrm{H}_{2}-2^{\prime}\right), 2.48\left(2 \mathrm{H}\right.$, s, dimethylglutaryl $\left.\mathrm{H}_{2}-4^{\prime}\right), 2.92,3.15$ (each $1 \mathrm{H}, \mathrm{d}, J=8.8$ $\mathrm{Hz}, \mathrm{H}_{2}-28$ ), 4.49 (1H, dd, $J=4.8,10.8 \mathrm{~Hz}, \mathrm{H}-3$ ), 4.54, 4.60 (each 1H, br s, $\left.\mathrm{H}_{2}-29\right), 7.23$ $(3 \mathrm{H}, \mathrm{t}, J=7.6 \mathrm{~Hz}$, trityl H-4'), $7.31(6 \mathrm{H}, \mathrm{t}, J=7.6 \mathrm{~Hz}$, trityl H-3',5'), $7.50(6 \mathrm{H}, \mathrm{d}, J=7.6 \mathrm{~Hz}$, trityl H-2',6'); ${ }^{13} \mathrm{C}$ NMR $\left(\mathrm{CDCl}_{3}, 100 \mathrm{MHz}\right) \delta 14.7$ (C-27), 15.9 (C-26), 16.1 (C-25), 16.5 (C-24), 18.2 (C-6), 19.1 (C-30), 20.8 (C-11), 23.8 (C-2), 25.2 (C-12), 27.0 (C-15), 27.9, 28.0 (dimethylglutaryl $\mathrm{CH}_{3}$ ), 28.0 (C-23), 30.0 (C-21), 30.2 (C-16), 32.7 (dimethylglutaryl C-3'), 34.2 (C-7), 35.2 (C-22), 37.1 (C-10), 37.3 (C-13), 37.7 (C-4), 38.4 (C-1), 40.7 (C-8), 42.5 (C-14), 45.2 (dimethylglutaryl C-4'), 45.7 (dimethylglutaryl C-2'), 47.6 (C-17), 47.8 (C-19), 
49.0 (C-18), 50.3 (C-9), 55.4 (C-5), 59.7 (C-28), 81.5 (C-3), 85.9 (trityl C( $\left.\mathrm{Ph})_{3}\right), 109.4$ (C-29), 126.8 (trityl C-4'), 127.7 (trityl C-2', 6'), 128.8 (trityl C-3', 5'), 144.5 (trityl C-1'), 150.8 (C-20), 172.3 (dimethylglutaryl COO-), 175.9 (dimethylglutaryl COOH). HRESIMS (positive) $\mathrm{m} / z 849.5403[\mathrm{M}+\mathrm{Na}]^{+}$(calcd for $\mathrm{C}_{56} \mathrm{H}_{74} \mathrm{O}_{5} \mathrm{Na}, 849.5434$ ).

3-O-Glutaryl-28-O-tritylbetulin (10): Yield 84.1\% (starting from $1.07 \mathrm{~g}$ of $\mathbf{7}$ and glutaric anhydride); white solid. $[\alpha]_{\mathrm{D}}^{25}+5.1^{\circ}\left(c 1.96, \mathrm{CHCl}_{3}\right) .{ }^{1} \mathrm{H} \mathrm{NMR}\left(\mathrm{CDCl}_{3}, 400 \mathrm{MHz}\right) \delta 0.53$ (3H, s, $\left.\mathrm{CH}_{3}-26\right), 0.79$ (3H, s, $\left.\mathrm{CH}_{3}-25\right), 0.83\left(3 \mathrm{H}, \mathrm{s}, \mathrm{CH}_{3}-24\right), 0.83\left(3 \mathrm{H}, \mathrm{s}, \mathrm{CH}_{3}-23\right), 0.90$ $\left(3 \mathrm{H}, \mathrm{s}, \mathrm{CH}_{3}-27\right), 1.65\left(3 \mathrm{H}, \mathrm{s}, \mathrm{CH}_{3}-30\right), 1.97\left(2 \mathrm{H}\right.$, quint, $J=7.2 \mathrm{~Hz}$, glutary $\left.\mathrm{H}_{2}-3^{\prime}\right), 2.15-2.24$ $(3 \mathrm{H}, \mathrm{m}, \mathrm{H}-22, \mathrm{H}-16$ and $\mathrm{H}-19), 2.39\left(2 \mathrm{H}, \mathrm{t}, J=7.2 \mathrm{~Hz}\right.$, glutary $\mathrm{H}_{2}-2$ '), $2.43(2 \mathrm{H}, \mathrm{s}$, glutaryl $\left.\mathrm{H}_{2}-4^{\prime}\right), 2.92,3.15$ (each $\left.1 \mathrm{H}, \mathrm{d}, J=8.8 \mathrm{~Hz}, \mathrm{H}_{2}-28\right), 4.48(1 \mathrm{H}, \mathrm{dd}, J=5.6,10.8 \mathrm{~Hz}, \mathrm{H}-3), 4.53$, 4.59 (each $1 \mathrm{H}$, br s, $\left.\mathrm{H}_{2}-29\right), 7.23(3 \mathrm{H}, \mathrm{t}, J=7.2 \mathrm{~Hz}$, trityl H-4'), $7.31(6 \mathrm{H}, \mathrm{t}, J=8.0,7.2 \mathrm{~Hz}$, trityl H-3',5'), $7.50\left(6 \mathrm{H}, \mathrm{d}, J=8.0 \mathrm{~Hz}\right.$, trityl H-2',6'); ${ }^{13} \mathrm{C} \mathrm{NMR}\left(\mathrm{CDCl}_{3}, 100 \mathrm{MHz}\right) \delta 14.7$ (C-27), 15.8 (C-26), 16.1 (C-25), 16.5 (C-24), 18.2 (C-6), 19.1 (C-30), 20.0 (glutaryl C-3'), 20.8 (C-11), 23.7 (C-2), 25.1 (C-12), 27.0 (C-15), 28.0 (C-23), 29.9 (C-21), 30.1 (C-16), 32.9 (glutaryl C-4'), 33.6 (glutaryl C-2'), 34.1 (C-7), 35.2 (C-22), 37.0 (C-10), 37.2 (C-13), 37.8 (C-4), 38.3 (C-1), 40.6 (C-8), 42.5 (C-14), 47.6 (C-17), 47.8 (C-19), 48.9 (C-18), 50.2 (C-9), 55.3 (C-5), 59.5 (C-28), 81.1 (C-3), 85.8 (trityl C(Ph) $)$ ), 109.4 (C-29), 126.8 (trityl C-4'), 127.7 (trityl C-2', 6'), 128.8 (trityl C-3', 5'), 144.5 (trityl C-1'), 150.8 (C-20), 172.6 (glutaryl $\mathrm{COO}^{-}$), 178.1 (glutaryl $\mathrm{COOH}$ ). HRESIMS (positive) $\mathrm{m} / z 821.5139[\mathrm{M}+\mathrm{Na}]^{+}$ (calcd for $\mathrm{C}_{54} \mathrm{H}_{70} \mathrm{O}_{5} \mathrm{Na}, 821.5121$ ).

4.2.3 General procedure for preparation of 3-O-acylbetulins (11-12)-3-OAcyl-28-O-tritylbetulin (1 equiv) was dissolved in EtOH- $\mathrm{CH}_{2} \mathrm{Cl}_{2}$ and then pyridium $p$ toluenesulfonate (PPTS) (3-5 equiv) was added. The reaction mixture was refluxed at $70^{\circ} \mathrm{C}$ overnight. After evaporation under reduced pressure, ice water was added to the residue, and extraction was performed with $\mathrm{CHCl}_{3}$. The organic layer was washed with water and brine, dried over $\mathrm{Na}_{2} \mathrm{SO}_{4}$, and concentrated. The residue was subjected over silica gel column (hexane/EtOAc=1:1) to give pure product.

3-O-(3',3'-Dimethylsuccinyl)betulin (11): Yield 58.9\% (starting from $1.51 \mathrm{~g}$ of 8); white solid; $[\alpha]_{\mathrm{D}}^{25}+24.1^{\circ}\left(c 1.95, \mathrm{CHCl}_{3}\right) .{ }^{1} \mathrm{H} \mathrm{NMR}\left(\mathrm{CDCl}_{3}, 400 \mathrm{MHz}\right) \delta 0.81\left(3 \mathrm{H}, \mathrm{s}, \mathrm{CH}_{3}-24\right)$, $0.84\left(6 \mathrm{H}, \mathrm{s}, \mathrm{CH}_{3}-23,25\right), 0.97$ (3H, s, $\left.\mathrm{CH}_{3}-27\right), 1.02\left(3 \mathrm{H}, \mathrm{s}, \mathrm{CH}_{3}-26\right), 1.28,1.30$ (each $3 \mathrm{H}$, s, dimethylsuccinyl $\left.\mathrm{CH}_{3}\right), 1.73\left(3 \mathrm{H}, \mathrm{s}, \mathrm{CH}_{3}-30\right), 2.38(1 \mathrm{H}, \mathrm{dt}, J=5.6,10.4 \mathrm{~Hz}, \mathrm{H}-19), 2.56$, 2.67 (each $1 \mathrm{H}, \mathrm{d}, J=15.6 \mathrm{~Hz}$, dimethylsuccinyl $\mathrm{H}_{2}-2^{\prime}$ ), 3.34, 3.80 (each $1 \mathrm{H}, \mathrm{d}, J=10.8 \mathrm{~Hz}$, $\mathrm{H}_{2}-28$ ), 4.49 (1H, dd, $J=5.2,10.4 \mathrm{~Hz}, \mathrm{H}-3$ ), 4.58, 4.68 (each $1 \mathrm{H}$, br s, $\mathrm{H}_{2}-29$ ); ${ }^{13} \mathrm{C}$ NMR $\left(\mathrm{CDCl}_{3}, 100 \mathrm{MHz}\right) \delta 14.7$ (C-27), $16.0(\mathrm{C}-26), 16.1$ (C-25), 16.5 (C-24), 18.2 (C-6), 19.0 (C-30), 20.8 (C-11), 23.6 (C-2), 25.1 (C-12), 25.0, 25.6 (dimethylsuccinyl $\mathrm{CH}_{3}$ ), 27.0 (C-15), 27.9 (C-23), 29.1 (C-16), 29.7 (C-21), 33.9 (C-22), 34.1 (C-7), 37.0 (C-10), 37.3 (C-13), 37.7 (C-4), 38.4 (C-1), 40.5 (dimethylsuccinyl C-3'), 40.9 (C-8), 42.7 (C-14), 44.7 (dimethylsuccinyl C-2'), 47.7 (C-17), 47.8 (C-19), 48.7 (C-18), 50.3 (C-9), 55.4 (C-5), 60.5 (C-28), 81.5 (C-3), 109.7 (C-29), 150.4 (C-20), 170.9 (dimethylsuccinyl COO-), 182.6 (dimethylsuccinyl COOH). HRESIMS (positive) $\mathrm{m} / z 593.4171[\mathrm{M}+\mathrm{Na}]^{+}$(calcd for $\mathrm{C}_{36} \mathrm{H}_{58} \mathrm{O}_{5} \mathrm{Na}$, 593.4182).

3-O-(3',3'-Dimethylglutaryl)betulin (12): Yield 67.2\% (starting from $1.0 \mathrm{~g}$ of 9); white solid. $[\alpha]_{\mathrm{D}}^{25}+24.0^{\circ}\left(c 1.88, \mathrm{CHCl}_{3}\right) .{ }^{1} \mathrm{H} \mathrm{NMR}\left(\mathrm{C}_{5} \mathrm{D}_{5} \mathrm{~N}, 400 \mathrm{MHz}\right) \delta 0.79\left(3 \mathrm{H}, \mathrm{s}, \mathrm{CH}_{3}-25\right)$, 0.92 (3H, s, $\left.\mathrm{CH}_{3}-24\right), 0.94$ (3H, s, $\left.\mathrm{CH}_{3}-23\right), 0.96$ (3H, s, $\left.\mathrm{CH}_{3}-26\right), 1.03$ (3H, s, $\left.\mathrm{CH}_{3}-27\right)$, 1.36, 1.37 (each $3 \mathrm{H}$, s, dimethylglutaryl $\left.\mathrm{CH}_{3}\right), 1.76\left(3 \mathrm{H}, \mathrm{s}, \mathrm{CH}_{3}-30\right), 2.11-2.17(1 \mathrm{H}, \mathrm{m}$, $\mathrm{H}-21), 2.37-2.45$ (2H, m, H-16 and H-22), 2.61 (1H, dt, $J=5.6,10.8 \mathrm{~Hz}, \mathrm{H}-19), 2.74,2.79$ (each $1 \mathrm{H}, \mathrm{d}, J=16.0 \mathrm{~Hz}$, dimethylglutaryl $\left.\mathrm{H}_{2}-2^{\prime}\right), 2.76\left(2 \mathrm{H}\right.$, s, dimethylglutaryl $\left.\mathrm{H}_{2}-4^{\prime}\right), 3.65$, 
4.06 (each 1H, d, $\left.J=10.8 \mathrm{~Hz}, \mathrm{H}_{2}-28\right), 4.71-4.74(1 \mathrm{H}, \mathrm{m}, \mathrm{H}-3), 4.74,4.88$ (each 1H, br s, $\left.\mathrm{H}_{2}-29\right) ;{ }^{13} \mathrm{C}$ NMR $\left(\mathrm{C}_{5} \mathrm{D}_{5} \mathrm{~N}, 100 \mathrm{MHz}\right) \delta 14.9(\mathrm{C}-27), 16.1(\mathrm{C}-26), 16.2(\mathrm{C}-25), 16.9(\mathrm{C}-24)$, 18.4 (C-6), 19.3 (C-30), 21.0 (C-11), 24.2 (C-2), 25.6 (C-12), 27.5 (C-15), 28.0 (dimethylglutaryl $\mathrm{CH}_{3}$ ), 28.1 (C-23), $30.0(\mathrm{C}-16), 30.4$ (C-21), 32.7 (dimethylglutaryl C-3'), 34.4 (C-7), 34.9 (C-22), 37.2 (C-10), 37.5 (C-13), 37.9 (C-4), 38.5 (C-1), 41.2 (C-8), 43.0 (C-14), 45.9 (dimethylglutaryl C-4', 2'), 48.3 (C-19), 48.5 (C-17), 49.1 (C-18), 50.5 (C-9), 55.5 (C-5), 59.4 (C-28), 80.6 (C-3), 109.9 (C-29), 151.2 (C-20), 171.9 (dimethylglutaryl COO-), 174.4 (dimethylglutaryl COOH). HRESIMS (positive) $\mathrm{m} / \mathrm{z} 607.4351[\mathrm{M}+\mathrm{Na}]^{+}$ (calcd for $\mathrm{C}_{37} \mathrm{H}_{60} \mathrm{O}_{5} \mathrm{Na}, 607.4338$ ).

4.2.4 3-O-Glutarylbetulin (13)-To a solution of $\mathbf{1 0}(1.50 \mathrm{~g}, 1.88 \mathrm{mmol})$ in $95 \% \mathrm{THF}$ $(50 \mathrm{~mL})$ was added anhydrous $\mathrm{FeCl}_{3}(1.22 \mathrm{~g}, 7.52 \mathrm{mmol})$. The solution was refluxed overnight. After cooling to room temperature, the solvent was evaporated in vacuo and the residue was dissolved in $\mathrm{CHCl}_{3}$. The $\mathrm{CHCl}_{3}$ solution was washed with water and brine, dried over $\mathrm{Na}_{2} \mathrm{SO}_{4}$, filtrated and concentrated. The crude product was purified by silica column chromatography (hexane/EtOAc $=2: 1)$ to give $\mathbf{1 3}$ as a white solid $(600 \mathrm{mg}$, yield

$57.4 \%) \cdot[\alpha]^{25}+25.1^{\circ}\left(c 3.45, \mathrm{CHCl}_{3}\right) .{ }^{1} \mathrm{H} \mathrm{NMR}\left(\mathrm{CDCl}_{3}, 400 \mathrm{MHz}\right) \delta 0.84\left(3 \mathrm{H}, \mathrm{s}, \mathrm{CH}_{3}-24\right)$, 0.85 (3H, s, $\left.\mathrm{CH}_{3}-23\right), 0.86$ (3H, s, $\left.\mathrm{CH}_{3}-25\right), 0.99$ (3H, s, $\left.\mathrm{CH}_{3}-27\right), 1.03$ (3H, s, $\left.\mathrm{CH}_{3}-26\right)$, $1.69\left(3 \mathrm{H}, \mathrm{s}, \mathrm{CH}_{3}-30\right), 1.98\left(2 \mathrm{H}\right.$, quint, $J=7.2 \mathrm{~Hz}$, glutary $\mathrm{H}_{2}-3$ '), 2.35-2.44 (1H, m, H-19), $2.39\left(2 \mathrm{H}, \mathrm{t}, J=7.2 \mathrm{~Hz}\right.$, glutary $\left.\mathrm{H}_{2}-2^{\prime}\right), 2.43\left(2 \mathrm{H}\right.$, s, glutaryl $\mathrm{H}_{2}-4$ '), 3.35, 3.81 (each $1 \mathrm{H}, \mathrm{d}, J$ $\left.=10.8 \mathrm{~Hz}, \mathrm{H}_{2}-28\right), 4.49(1 \mathrm{H}, \mathrm{dd}, J=5.6,10.4 \mathrm{~Hz}, \mathrm{H}-3), 4.59,4.69$ (each $1 \mathrm{H}, \mathrm{br} \mathrm{s}$, $\left.\mathrm{H}_{2}-29\right) ;{ }^{13} \mathrm{C}$ NMR $\left(\mathrm{CDCl}_{3}, 100 \mathrm{MHz}\right) \delta 14.7$ (C-27), 16.0 (C-26), 16.1 (C-25), 16.5 (C-24), 18.2 (C-6), 19.1 (C-30), 20.1 (glutaryl C-3'), 20.9 (C-11), 23.7 (C-2), 25.2 (C-12), 27.1 (C-15), 28.0 (C-23), 29.2 (C-16), 29.8 (C-21), 32.9 (glutaryl C-4'), 33.7 (glutaryl C-2'), 34.0 (C-22), 34.2 (C-7), 37.1 (C-10), 37.3 (C-13), 37.8 (C-4), 38.4 (C-1), 41.0 (C-8), 42.7 (C-14), 47.8 (C-17), 47.8 (C-19), 48.8 (C-18), 50.3 (C-9), 55.4 (C-5), 60.6 (C-28), 81.1 (C-3), 109.7 (C-29), 150.4 (C-20), 172.6 (glutaryl COO-), 177.6 (glutaryl COOH). HRESIMS (positive) $m / z 579.4027[\mathrm{M}+\mathrm{Na}]^{+}$(calcd for $\mathrm{C}_{35} \mathrm{H}_{56} \mathrm{O}_{5} \mathrm{Na}, 579.4025$ ).

4.2.5 General procedure of hydrogenation-3-O-Acyl-dihydrobetulin derivatives $(4$, 14, and 15) were prepared by treating a solution of 3-O-acylbetulin derivative (250-400 $\mathrm{mg}$ ) in EtOAc-EtOH with $10 \%$ palladium catalyst (Pd-C, $240-800 \mathrm{mg}$ ) under hydrogen atmosphere overnight with stirring. The reaction mixture was filtered, and the filtrate was concentrated under reduced pressure to give a white solid. The purity of the product was confirmed by NMR analyses and used in the next reaction without further purification.

3-O-(3',3'-Dimethylsuccinyl)dihydrobetulin (14): Yield 100\% (starting from $395 \mathrm{mg}$ of $\mathbf{1 1}$ and $240 \mathrm{mg}$ of $10 \% \mathrm{Pd}-\mathrm{C})$; white solid; $[\alpha]_{\mathrm{D}}^{24}-9.3^{\circ}\left(c 1.5, \mathrm{CHCl}_{3}\right) .{ }^{1} \mathrm{H} \mathrm{NMR}\left(\mathrm{C}_{5} \mathrm{D}_{5} \mathrm{~N}, 400\right.$ MHz) $\delta 0.79$ (6H, s, $\left.\mathrm{CH}_{3}-25\right), 0.82,0.89$ (each $\left.2 \mathrm{H}, \mathrm{d}, J=8.0 \mathrm{~Hz}, \mathrm{CH}_{3}-29,30\right), 0.94$ (3H, s, $\left.\mathrm{CH}_{3}-24\right), 0.96$ (3H, s, CH3-23, 26), 0.98 (3H, s, $\left.\mathrm{CH}_{3}-27\right), 1.53$ (6H, s, dimethylsuccinyl $\left.\mathrm{CH}_{3}\right), 2.38-2.41(2 \mathrm{H}, \mathrm{m}, \mathrm{H}-16$ and $\mathrm{H}-22), 2.88,2.94$ (each $1 \mathrm{H}, \mathrm{d}, J=15.6 \mathrm{~Hz}$, dimethylsuccinyl $\mathrm{H}_{2}-2$ ), 3.58, 4.04 (each $\left.1 \mathrm{H}, \mathrm{d}, J=10.4 \mathrm{~Hz}, \mathrm{H}_{2}-28\right), 4.76$ (1H, dd, $J=4.4$, $11.6 \mathrm{~Hz}, \mathrm{H}-3) ;{ }^{13} \mathrm{C}$ NMR $\left(\mathrm{C}_{5} \mathrm{D}_{5} \mathrm{~N}, 100 \mathrm{MHz}\right) \delta 14.8$ (C-27), 15.2 (C-29), 16.1 (C-26), 16.2 (C-25), 16.9 (C-24), 18.4 (C-6), 21.1 (C-11), 22.3 (C-21), 23.2 (C-30), 24.1 (C-2), 25.9, 26.2 (dimethylsuccinyl $\mathrm{CH}_{3}$ ), 27.2 (C-12), 27.4 (C-15), 28.1 (C-23), 29.8 (C-20), 30.1 (C-16), 34.5 (C-7), 34.9 (C-22), 37.0 (C-13), 37.2 (C-10), 38.0 (C-4), 38.5 (C-1), 40.9 (dimethylsuccinyl C-3'), 41.2 (C-8), 43.1 (C-14), 45.0 (C-19), 45.2 (dimethylsuccinyl C-2'), 48.6 (C-17), 48.4 (C-18), 50.1 (C-9), 55.5 (C-5), 59.4 (C-28), 80.9 (C-3), 171.6 (dimethylsuccinyl $\mathrm{COO}^{-}$), 179.3 (dimethylsuccinyl COOH). HRESIMS (positive) $\mathrm{m} / \mathrm{z}$ $595.4327[\mathrm{M}+\mathrm{Na}]^{+}$(calcd for $\mathrm{C}_{36} \mathrm{H}_{60} \mathrm{O}_{5} \mathrm{Na}, 595.4338$ ). 
3-O-(3',3'-Dimethylglutaryl)dihydrobetulin (15): Yield 97.4\% (starting from $250 \mathrm{mg}$ of 12 and $250 \mathrm{mg}$ of $10 \% \mathrm{Pd}-\mathrm{C})$; white solid. $[\alpha]_{\mathrm{D}}^{25}-8.9^{\circ}\left(c 1.54, \mathrm{CHCl}_{3}\right) .{ }^{1} \mathrm{H} \mathrm{NMR}\left(\mathrm{C}_{5} \mathrm{D}_{5} \mathrm{~N}\right.$, $400 \mathrm{MHz}) \delta 0.82\left(3 \mathrm{H}, \mathrm{s}, \mathrm{CH}_{3}-25\right), 0.83,0.90\left(\right.$ each $\left.2 \mathrm{H}, \mathrm{d}, J=8.0 \mathrm{~Hz}, \mathrm{CH}_{3}-29,30\right), 0.93$ (3H, s, $\left.\mathrm{CH}_{3}-24\right), 0.95$ ( $\left.3 \mathrm{H}, \mathrm{s}, \mathrm{CH}_{3}-23\right), 0.97$ ( $\left.3 \mathrm{H}, \mathrm{s}, \mathrm{CH}_{3}-26\right), 1.00$ (3H, s, $\left.\mathrm{CH}_{3}-27\right), 1.37$, 1.37 (each $3 \mathrm{H}$, s, dimethylglutaryl $\mathrm{CH}_{3}$ ), 2.37-2.41 (2H, m, H-16 and $\left.\mathrm{H}-22\right), 2.75,2.79$ (each $1 \mathrm{H}, \mathrm{d}, J=14.4 \mathrm{~Hz}$, dimethylglutaryl $\left.\mathrm{H}_{2}-2^{\prime}\right), 2.77\left(2 \mathrm{H}, \mathrm{s}\right.$, dimethylglutaryl $\left.\mathrm{H}_{2}-4^{\prime}\right), 3.59$, 4.05 (each $\left.1 \mathrm{H}, \mathrm{d}, J=10.8 \mathrm{~Hz}, \mathrm{H}_{2}-28\right), 4.74(1 \mathrm{H}, \mathrm{dd}, J=4.4,11.6 \mathrm{~Hz}, \mathrm{H}-3) ;{ }^{13} \mathrm{C}$ NMR $\left(\mathrm{C}_{5} \mathrm{D}_{5} \mathrm{~N}, 100 \mathrm{MHz}\right) \delta 14.8(\mathrm{C}-27), 15.2(\mathrm{C}-29), 16.1(\mathrm{C}-26), 16.2(\mathrm{C}-25), 16.9(\mathrm{C}-24), 18.4$ (C-6), 21.1 (C-11), 22.3 (C-21), 23.2 (C-30), 24.2 (C-2), 27.2 (C-12), 27.4 (C-15), 28.0 (dimethylglutaryl $\mathrm{CH}_{3}$ ), 28.1 (C-23), 29.8 (C-20), 30.1 (C-16), 32.7 (dimethylglutaryl C-3'), 34.5 (C-7), 34.9 (C-22), 37.0 (C-13), $37.2(\mathrm{C}-10), 37.9$ (C-4), 38.5 (C-1), 41.2 (C-8), 43.1 (C-14), 45.0 (C-19), 45.9 (dimethylglutaryl C-4', 2'), 48.4 (C-18), 48.6 (C-17), 50.2 (C-9), 55.5 (C-5), 59.4 (C-28), 80.7 (C-3), 172.0 (dimethylglutaryl COO-), 174.4 (dimethylglutaryl COOH). HRESIMS (positive) $\mathrm{m} / \mathrm{z} 609.4493[\mathrm{M}+\mathrm{Na}]^{+}$(calcd for $\left.\mathrm{C}_{37} \mathrm{H}_{62} \mathrm{O}_{5} \mathrm{Na}, 609.4495\right)$.

3-O-Glutaryl-dihydrobetulin (4): Yield $91.1 \%$ (starting from $387 \mathrm{mg}$ of $\mathbf{1 3}$ and $800 \mathrm{mg}$ of $10 \%$ Pd-C); white solid. $[\alpha]_{\mathrm{D}}^{25}-5.4^{\circ}\left(c 2.40, \mathrm{CHCl}_{3}\right) .{ }^{1} \mathrm{H} \mathrm{NMR}\left(\mathrm{CDCl}_{3}, 400 \mathrm{MHz}\right) \delta 0.78$, 0.85 (each $\left.2 \mathrm{H}, \mathrm{d}, J=8.0 \mathrm{~Hz}, \mathrm{CH}_{3}-29,30\right), 0.85\left(3 \mathrm{H}, \mathrm{s}, \mathrm{CH}_{3}-24\right), 0.85\left(3 \mathrm{H}, \mathrm{s}, \mathrm{CH}_{3}-23\right), 0.87$ $\left(3 \mathrm{H}, \mathrm{s}, \mathrm{CH}_{3}-25\right), 0.96\left(3 \mathrm{H}, \mathrm{s}, \mathrm{CH}_{3}-27\right), 1.04\left(3 \mathrm{H}, \mathrm{s}, \mathrm{CH}_{3}-26\right), 1.99(2 \mathrm{H}$, quint, $J=7.2 \mathrm{~Hz}$, glutary $\left.\mathrm{H}_{2}-3^{\prime}\right), 2.40\left(2 \mathrm{H}, \mathrm{t}, J=7.2 \mathrm{~Hz}\right.$, glutary $\left.\mathrm{H}_{2}-2^{\prime}\right), 2.43\left(2 \mathrm{H}\right.$, s, glutaryl $\left.\mathrm{H}_{2}-4^{\prime}\right), 3.32,3.79$ (each $\left.1 \mathrm{H}, \mathrm{d}, J=\mathrm{Hz}, \mathrm{H}_{2}-28\right), 4.50(1 \mathrm{H}, \mathrm{dd}, J=6.4,10.0 \mathrm{~Hz}, \mathrm{H}-3) ;{ }^{13} \mathrm{C} \mathrm{NMR}\left(\mathrm{CDCl}_{3}, 100\right.$ MHz) $\delta$ 14.6 (C-27), 14.9 (C-29), 16.0 (C-26), 16.1 (C-25), 16.6 (C-24), 18.2 (C-6), 20.1 (glutaryl C-3'), 20.9 (C-11), 21.7 (C-21), 22.9 (C-30), 23.7 (C-2), 26.9 (C-12), 26.9 (C-15), 28.0 (C-23), 29.3 (C-16), 29.5 (C-20), 33.0 (glutaryl C-4'), 33.7 (glutaryl C-2'), 34.0 (C-22), 34.3 (C-7), 36.9 (C-13), 37.1 (C-10), 37.8 (C-4), 38.4 (C-1), 41.0 (C-8), 42.9 (C-14), 44.6 (C-19), 47.9 (C-17), 48.1 (C-18), 50.0 (C-9), 55.4 (C-5), 60.6 (C-28), 81.1 (C-3), 172.7 (glutaryl COO-), 177.9 (glutaryl COOH). HRESIMS (positive) $\mathrm{m} / z 581.4180[\mathrm{M}+\mathrm{Na}]^{+}$ (calcd for $\mathrm{C}_{35} \mathrm{H}_{58} \mathrm{O}_{5} \mathrm{Na}, 581.4182$ ).

\subsubsection{General procedure for syntheses of 3-O-acylbetulin benzhydryl ester derivatives (16-20)-To a solution of 3- $O$-acylbetulin derivatives ( 1 equiv) in $\mathrm{CH}_{2} \mathrm{Cl}_{2}$ - $\mathrm{CH}_{3} \mathrm{OH}$, freshly prepared diphenyldiazomethane was added until the reaction solution turned pink. The resulting mixture was kept stirring at room temperature overnight. After evaporated solvent in vacuo, the residue was subjected over silica gel column (hexane/ $\mathrm{EtOAc}=10: 1)$ to give the desired product.}

3-O-(3',3'-Dimethylsuccinyl)betulin benzhydryl ester (16): Yield 100\% (starting from $265 \mathrm{mg}$ of 11); white solid; $[\alpha]_{\mathrm{D}}^{17}+16.9^{\circ}\left(c 1.74, \mathrm{CHCl}_{3}\right) .{ }^{1} \mathrm{H} \mathrm{NMR}\left(\mathrm{CDCl}_{3}, 400 \mathrm{MHz}\right) \delta$ 0.75 (3H, s, $\left.\mathrm{CH}_{3}-24\right), 0.79$ (3H, s, $\left.\mathrm{CH}_{3}-23\right), 0.80$ (3H, s, $\left.\mathrm{CH}_{3}-25\right), 0.99$ (3H, s, $\left.\mathrm{CH}_{3}-27\right)$, $1.03\left(3 \mathrm{H}, \mathrm{s}, \mathrm{CH}_{3}-26\right), 1.32,1.32$ (each $3 \mathrm{H}$, s, dimethylsuccinyl $\left.\mathrm{CH}_{3}\right), 1.70\left(3 \mathrm{H}, \mathrm{s}, \mathrm{CH}_{3}-30\right)$, $2.40(1 \mathrm{H}, \mathrm{dt}, J=5.6,10.4 \mathrm{~Hz}, \mathrm{H}-19), 2.66,2.70$ (each $1 \mathrm{H}, \mathrm{d}, J=16.0 \mathrm{~Hz}$, dimethylsuccinyl $\left.\mathrm{H}_{2}-2^{\prime}\right), 3.34,3.80$ (each $\left.1 \mathrm{H}, \mathrm{d}, J=10.8 \mathrm{~Hz}, \mathrm{H}_{2}-28\right), 4.45$ (1H, dd, $J=5.2,10.8 \mathrm{~Hz}, \mathrm{H}-3$ ), 4.60, 4.70 (each $1 \mathrm{H}$, br s, $\left.\mathrm{H}_{2}-29\right), 6.87\left(1 \mathrm{H}, \mathrm{s}, \mathrm{CH}(\mathrm{Ph})_{2}\right), 7.25-7.34(10 \mathrm{H}, \mathrm{m}$, aromatic$\mathrm{H}) ;{ }^{13} \mathrm{C} \mathrm{NMR}\left(\mathrm{CDCl}_{3}, 100 \mathrm{MHz}\right) \delta 14.7$ (C-27), 15.9 (C-26), 16.0 (C-25), 16.4 (C-24), 18.1 (C-6), 19.0 (C-30), 20.8 (C-11), 23.5 (C-2), 25.1 (C-12), 25.1, 25.4 (dimethylsuccinyl $\mathrm{CH}_{3}$ ), 27.0 (C-15), 27.8 (C-23), 29.1 (C-16), 29.7 (C-21), 33.9 (C-22), 34.1 (C-7), 37.0 (C-10), 37.2 (C-13), 37.6 (C-4), 38.3 (C-1), 40.6 (dimethylsuccinyl C-3'), 40.9 (C-8), 42.6 (C-14), 44.5 (dimethylsuccinyl C-2'), 47.7 (C-17), 47.8 (C-19), 48.7 (C-18), 50.2 (C-9), 55.3 (C-5), $60.4(\mathrm{C}-28), 76.9\left(\mathrm{CH}(\mathrm{Ph})_{2}\right), 81.2(\mathrm{C}-3), 109.7$ (C-29), 127.0 (benzhydryl C-2', 6'), 127.7 (benzyhydryl C-4'), 128.3 (benzhydryl C-3', 5'), 140.3 (benzhydryl C-1'), 150.4 (C-20), 
170.8 (dimethylsuccinyl 1'-COO-), 175.3 (dimethylsuccinyl 4'-COO-). HRESIMS (positive) $\mathrm{m} / z$. $759.4958[\mathrm{M}+\mathrm{Na}]^{+}$(calcd for $\mathrm{C}_{49} \mathrm{H}_{68} \mathrm{O}_{5} \mathrm{Na}, 759.4964$ ).

3-O-(3',3'-Dimethyglutaryl)betulin benzhydryl ester (17): Yield 100\% (starting from 207 mg of 12); white solid. $[\alpha]_{\mathrm{D}}^{27}+18.6^{\circ}\left(c 1.29, \mathrm{CHCl}_{3}\right) .{ }^{1} \mathrm{H} \mathrm{NMR}\left(\mathrm{CDCl}_{3}, 400 \mathrm{MHz}\right) \delta 0.81$ (3H, s, $\left.\mathrm{CH}_{3}-24\right), 0.84$ (6H, s, $\left.\mathrm{CH}_{3}-23,25\right), 0.99$ (3H, s, $\left.\mathrm{CH}_{3}-27\right), 1.03$ (3H, s, $\left.\mathrm{CH}_{3}-26\right), 1.09$ (6H, s, dimethylglutaryl $\left.\mathrm{CH}_{3}\right), 1.70\left(3 \mathrm{H}, \mathrm{s}, \mathrm{CH}_{3}-30\right), 2.34-2.45(1 \mathrm{H}, \mathrm{m}, \mathrm{H}-19), 2.36,2.43$ (each $1 \mathrm{H}, \mathrm{d}, J=14.4 \mathrm{~Hz}$, dimethylglutaryl $\mathrm{H}_{2}-2^{\prime}$ ), 2.55, 2.58 (each $1 \mathrm{H}, \mathrm{d}, J=14.4 \mathrm{~Hz}$, dimethylglutaryl $\mathrm{H}_{2}-4$ '), 3.34, 3.81 (each $1 \mathrm{H}, \mathrm{d}, J=10.8 \mathrm{~Hz}, \mathrm{H}_{2}-28$ ), 4.47 (1H, dd, $J=4.8$, $11.2 \mathrm{~Hz}, \mathrm{H}-3), 4.60,4.70$ (each $1 \mathrm{H}$, br s, $\left.\mathrm{H}_{2}-29\right), 6.89\left(1 \mathrm{H}, \mathrm{s}, \mathrm{CH}(\mathrm{Ph})_{2}\right), 7.25-7.39(10 \mathrm{H}, \mathrm{m}$, aromatic-H); ${ }^{13} \mathrm{C}$ NMR $\left(\mathrm{CDCl}_{3}, 100 \mathrm{MHz}\right) \delta 14.7(\mathrm{C}-27), 16.0(\mathrm{C}-26), 16.1(\mathrm{C}-25), 16.6$ (C-24), 18.2 (C-6), 19.0 (C-30), 20.8 (C-11), 23.8 (C-2), 25.2 (C-12), 27.0 (C-15), 27.6, 27.7 (dimethylglutaryl $\mathrm{CH}_{3}$ ), 28.0 (C-23), 29.2 (C-16), 29.7 (C-21), 32.8 (dimethylglutaryl C-3'), 33.9 (C-22), 34.1 (C-7), 37.0 (C-10), 37.3 (C-13), 37.7 (C-4), 38.4 (C-1), 40.9 (C-8), 42.7 (C-14), 45.5 (dimethylsuccinyl C-4'), 45.8 (dimethylsuccinyl C-2'), 47.8 (C-17), 47.8 (C-19), 48.7 (C-18), 50.3 (C-9), 55.4 (C-5), 60.5 (C-28), 76.7 (CH(Ph) 2 ), 80.9 (C-3), 109.7 (C-29), 127.1 (benzhydryl C-2', 6'), 127.8 (benzyhydryl C-4'), 128.4 (benzhydryl C-3', 5'), 140.2, 140.3 (benzhydryl C-1'), 150.4 (C-20), 170.8 (dimethylglutaryl 5'-COO-), 171.6 (dimethylglutaryl 1'-COO-). HRESIMS (positive) $\mathrm{m} / z, 773.5124[\mathrm{M}+\mathrm{Na}]^{+}$(calcd for $\left.\mathrm{C}_{50} \mathrm{H}_{70} \mathrm{O}_{5} \mathrm{Na}, 773.5121\right)$.

3-O-(3',3'-Dimethylsuccinyl)dihydrobetulin benzhydryl ester (18): Yield 100\% (starting from $400 \mathrm{mg}$ of 14); white solid. $[\alpha]_{\mathrm{D}}^{25}-7.9^{\circ}\left(c 5.27, \mathrm{CHCl}_{3}\right) .{ }^{1} \mathrm{H} \mathrm{NMR}\left(\mathrm{CDCl}_{3}, 400 \mathrm{MHz}\right)$ $\delta 0.75\left(3 \mathrm{H}, \mathrm{s}, \mathrm{CH}_{3}-24\right), 0.79\left(3 \mathrm{H}, \mathrm{s}, \mathrm{CH}_{3}-23\right), 0.78,0.85$ (each $2 \mathrm{H}, \mathrm{d}, J=8.0 \mathrm{~Hz}, \mathrm{CH}_{3}-29$, 30), $0.81\left(3 \mathrm{H}, \mathrm{s}, \mathrm{CH}_{3}-25\right), 0.96\left(3 \mathrm{H}, \mathrm{s}, \mathrm{CH}_{3}-27\right), 1.03\left(3 \mathrm{H}, \mathrm{s}, \mathrm{CH}_{3}-26\right), 1.31,1.32$ (each $3 \mathrm{H}$, s, dimethylsuccinyl $\mathrm{CH}_{3}$ ), 2.66, 2.70 (each $1 \mathrm{H}, \mathrm{d}, J=15.6 \mathrm{~Hz}$, dimethylsuccinyl $\mathrm{H}_{2}-2^{\prime}$ ), 3.31, 3.78 (each 1H, d, $\left.J=10.8 \mathrm{~Hz}, \mathrm{H}_{2}-28\right), 4.45(1 \mathrm{H}, \mathrm{dd}, J=5.0,10.8 \mathrm{~Hz}, \mathrm{H}-3), 6.87(1 \mathrm{H}$, s, $\left.\mathrm{CH}(\mathrm{Ph})_{2}\right), 7.27-7.33\left(10 \mathrm{H}, \mathrm{m}\right.$, aromatic-H); ${ }^{13} \mathrm{C} \mathrm{NMR}\left(\mathrm{CDCl}_{3}, 100 \mathrm{MHz}\right) \delta 14.6(\mathrm{C}-27)$, 14.9 (C-29), 15.9 (C-26), 16.0 (C-25), 16.5 (C-24), 18.1 (C-6), 20.8 (C-11), 21.7 (C-21), 22.9 (C-30), 23.5 (C-2), 25.2, 25.4 (dimethylsuccinyl $\mathrm{CH}_{3}$ ), 26.8 (C-12), 26.9 (C-15), 27.9 (C-23), 29.3 (C-16), 29.5 (C-20), 34.0 (C-22), 34.2 (C-7), 36.8 (C-13), 37.0 (C-10), 37.6 (C-4), 38.3 (C-1), 40.7 (dimethylsuccinyl C-3'), 40.9 (C-8), 42.8 (C-14), 44.5 (C-19 and dimethylsuccinyl C-2'), 47.9 (C-17), 48.0 (C-18), 49.9 (C-9), 55.3 (C-5), 60.6 (C-28), 77.0 $\left(\mathrm{CH}(\mathrm{Ph})_{2}\right), 81.3(\mathrm{C}-3), 127.0$ (benzhydryl C-2', 6'), 127.7 (benzyhydryl C-4'), 128.4 (benzhydryl C-3', 5'), 140.3 (benzhydryl C-1'), 170.8 (dimethylsuccinyl 1'-COO-), 175.4 (dimethylsuccinyl 4'-COO-). HRESIMS (positive) $\mathrm{m} / 2761.5118[\mathrm{M}+\mathrm{Na}]^{+}$(calcd for $\left.\mathrm{C}_{49} \mathrm{H}_{70} \mathrm{O}_{5} \mathrm{Na}, 761.5121\right)$.

3-O-(3',3'-Dimethylglutaryl)dihydrobetulin benzhydryl ester (19): Yield 85.3\% (starting from $228 \mathrm{mg}$ of 15); white solid. $[\alpha]_{\mathrm{D}}^{24}-7.7^{\circ}\left(c 1.26, \mathrm{CHCl}_{3}\right) .{ }^{1} \mathrm{H} \mathrm{NMR}\left(\mathrm{CDCl}_{3}, 400 \mathrm{MHz}\right)$ $\delta 0.79\left(3 \mathrm{H}, \mathrm{s}, \mathrm{CH}_{3}-24\right), 0.82\left(3 \mathrm{H}, \mathrm{s}, \mathrm{CH}_{3}-23,25\right), 0.76,0.83$ (each $2 \mathrm{H}, \mathrm{d}, J=8.0 \mathrm{~Hz}$, $\left.\mathrm{CH}_{3}-29,30\right), 0.94\left(3 \mathrm{H}, \mathrm{s}, \mathrm{CH}_{3}-27\right), 1.00\left(3 \mathrm{H}, \mathrm{s}, \mathrm{CH}_{3}-26\right), 1.07$ (6H, s, dimethylglutaryl $\mathrm{CH}_{3}$ ), 2.34, 2.41 (each $1 \mathrm{H}, \mathrm{d}, J=14.4 \mathrm{~Hz}$, dimethylglutaryl $\mathrm{H}_{2}-2$ ) $)$, 2.53, 2.56 (each $1 \mathrm{H}, \mathrm{d}, J$ $=14.4 \mathrm{~Hz}$, dimethylglutaryl $\left.\mathrm{H}_{2}-4^{\prime}\right), 3.30,3.76\left(\right.$ each $\left.1 \mathrm{H}, \mathrm{d}, J=10.8 \mathrm{~Hz}, \mathrm{H}_{2}-28\right), 4.46(1 \mathrm{H}$, $\mathrm{dd}, J=4.8,10.8 \mathrm{~Hz}, \mathrm{H}-3), 6.87\left(1 \mathrm{H}, \mathrm{s}, \mathrm{CH}(\mathrm{Ph})_{2}\right), 7.23-7.34\left(10 \mathrm{H}, \mathrm{m}\right.$, aromatic-H); ${ }^{13} \mathrm{C}$ NMR $\left(\mathrm{CDCl}_{3}, 100 \mathrm{MHz}\right) \delta 14.5$ (C-27), 14.8 (C-29), 15.9 (C-26), 16.0 (C-25), 16.5 (C-24), 18.1 (C-6), 20.7 (C-11), 21.6 (C-21), 22.9 (C-30), 23.7 (C-2), 26.8 (C-12), 26.8 (C-15), 27.5, 27.6 (dimethylglutaryl $\mathrm{CH}_{3}$ ), 27.9 (C-23), 29.3 (C-16), 29.4 (C-20), 32.7 (dimethylglutaryl C-3'), 34.0 (C-22), 34.1 (C-7), 36.7 (C-13), 36.9 (C-10), 37.6 (C-4), 38.3 (C-1), , 40.9 (C-8), 42.8 (C-14), 44.5 (C-19), 45.3 (dimethylglutaryl C-4'), 45.7 (dimethylglutaryl C-2'), 47.8 (C-17), 48.0 (C-18), 49.9 (C-9), 55.2 (C-5), 60.1 (C-28), 76.6 (CH(Ph) 2 ), 80.8 (C-3), 127.0 
(benzhydryl C-2', 6'), 127.7 (benzyhydryl C-4'), 128.3 (benzhydryl C-3', 5'), 140.2 (benzhydryl C-1'), 170.7 (dimethylglutaryl 5'-COO-), 171.5 (dimethylglutaryl 1-cOO-). ${ }_{4} R_{\text {essms }}$ (positive) $\mathrm{m} / z 775.5303[\mathrm{M}+\mathrm{Na}]^{+}$(calcd for $\mathrm{C}_{50} \mathrm{H}_{72} \mathrm{O}_{5} \mathrm{Na}, 775.5277$ ).

3-O-Glutaryl-dihydrobetulin benzhydryl ester (20): Yield 96.4\% (starting from $332 \mathrm{mg}$ of 4); white solid. $[\alpha]_{\mathrm{D}}^{27}-4.2^{\circ}\left(c 3.84, \mathrm{CHCl}_{3}\right) .{ }^{1} \mathrm{H} \mathrm{NMR}\left(\mathrm{CDCl}_{3}, 400 \mathrm{MHz}\right) \delta 0.84(3 \mathrm{H}, \mathrm{s}$, $\left.\mathrm{CH}_{3}-24\right), 0.85$ (3H, s, $\left.\mathrm{CH}_{3}-23\right), 0.79,0.85$ (each $\left.2 \mathrm{H}, \mathrm{d}, J=8.0 \mathrm{~Hz}, \mathrm{CH}_{3}-29,30\right), 0.87$ (3H, s, $\left.\mathrm{CH}_{3}-25\right), 0.97\left(3 \mathrm{H}, \mathrm{s}, \mathrm{CH}_{3}-27\right), 1.03\left(3 \mathrm{H}, \mathrm{s}, \mathrm{CH}_{3}-26\right), 2.00(2 \mathrm{H}, \mathrm{t}, J=7.2 \mathrm{~Hz}$, glutaryl $\left.\mathrm{H}_{2}-3^{\prime}\right), 2.36\left(2 \mathrm{H}, \mathrm{t}, J=7.2 \mathrm{~Hz}\right.$, glutaryl $\left.\mathrm{H}_{2}-2^{\prime}\right), 2.51\left(2 \mathrm{H}, \mathrm{t}, J=7.2 \mathrm{~Hz}\right.$, glutaryl $\left.\mathrm{H}_{2}-4^{\prime}\right), 3.32$, 3.78 (each $\left.1 \mathrm{H}, \mathrm{d}, J=10.8 \mathrm{~Hz}, \mathrm{H}_{2}-28\right), 4.50(1 \mathrm{H}, \mathrm{dd}, J=5.6,10.4 \mathrm{~Hz}, \mathrm{H}-3), 6.90(1 \mathrm{H}, \mathrm{s}$, $\left.\mathrm{CH}(\mathrm{Ph})_{2}\right), 7.26-7.34\left(10 \mathrm{H}, \mathrm{m}\right.$, aromatic-H); ${ }^{13} \mathrm{C} \mathrm{NMR}\left(\mathrm{CDCl}_{3}, 100 \mathrm{MHz}\right) \delta 14.6(\mathrm{C}-27)$, 14.9 (C-29), 16.0 (C-26), 16.1 (C-25), 16.5 (C-24), 18.2 (C-6), 20.4 (glutaryl C-3'), 20.9 (C-11), 21.7 (C-21), 22.9 (C-30), 23.7 (C-2), 26.9 (C-12), 26.9 (C-15), 28.0 (C-23), 29.3 (C-16), 29.5 (C-20), 33.6 (glutaryl C-4'), 33.7 (glutaryl C-2'), 34.0 (C-22), 34.3 (C-7), 36.8 (C-13), 37.1 (C-10), 37.8 (C-4), 38.4 (C-1), 41.0 (C-8), 42.9 (C-14), 44.6 (C-19), 47.9 (C-17), 48.1 (C-18), 50.0 (C-9), 55.3 (C-5), $60.6(\mathrm{C}-28), 76.9\left(\mathrm{CH}(\mathrm{Ph})_{2}\right), 81.0(\mathrm{C}-3), 127.1$ (benzhydryl C-2', 6'), 127.9 (benzyhydryl C-4'), 128.5 (benzhydryl C-3', 5'), 140.2 (benzhydryl C-1'), 171.9 (glutaryl 5'-COO-), 172.6 (glutaryl 1'-COO-). HRESIMS (positive) $\mathrm{m} / z 747.4962[\mathrm{M}+\mathrm{Na}]^{+}$(calcd for $\mathrm{C}_{48} \mathrm{H}_{68} \mathrm{O}_{5} \mathrm{Na}, 747.4964$ ).

\subsubsection{General procedure for syntheses of 3,28-di-O-acylbetulin derivatives}

(21-34)-3,28-Di- $O$-acylbetulin derivatives were prepared by refluxing a solution of 3-Oacylbetulin benzhydryl ester derivatives (1 equiv), DMAP (1.5-4.5 equiv) and appropriate dicarboxylic acid (6-9 equiv) or anhydride (3-6 equiv) in anhydrous pyridine (5-8 $\mathrm{mL}$ ) overnight. After cooling to room temperature, the reaction mixture was poured into icewater, and extracted with $\mathrm{CHCl}_{3}$. The organic layer was washed with water, $2 \mathrm{~N} \mathrm{HCl}$ solution and brine in turn, dried over $\mathrm{Na}_{2} \mathrm{SO}_{4}$, and concentrated. The residue was chromatographed over silica gel column $\left(\mathrm{CHCl}_{3}\right.$ or hexane/EtOAc $\left.=5: 1\right)$ or purified by preparative HPLC.

\section{3-O-(4'-Benzhydryloxy-3',3'-dimethylsuccinyl)-28-O-succinylbetulin (21): Yield 76.8\%}

(starting from $504.6 \mathrm{mg}$ of $\mathbf{1 6}$ and succinic anhydride); white solid. $[\alpha]_{\mathrm{D}}^{18}+5.6^{\circ}(c 0.5$, $\left.\mathrm{CHCl}_{3}\right) .{ }^{1} \mathrm{H} \mathrm{NMR}\left(\mathrm{CDCl}_{3}, 500 \mathrm{MHz}\right) \delta 0.73\left(3 \mathrm{H}, \mathrm{s}, \mathrm{CH}_{3}-24\right), 0.77\left(6 \mathrm{H}, \mathrm{s}, \mathrm{CH}_{3}-23\right.$ and $\left.\mathrm{CH}_{3}-25\right), 0.96\left(3 \mathrm{H}, \mathrm{s}, \mathrm{CH}_{3}-27\right), 1.01\left(3 \mathrm{H}, \mathrm{s}, \mathrm{CH}_{3}-26\right), 1.30,1.31$ (each $3 \mathrm{H}, \mathrm{s}$, dimethylsuccinyl $\left.\mathrm{CH}_{3}\right), 1.68\left(3 \mathrm{H}, \mathrm{s}, \mathrm{CH}_{3}-30\right), 2.43(1 \mathrm{H}, \mathrm{dt}, J=5.5,10.9 \mathrm{~Hz}, \mathrm{H}-19), 2.64$, 2.69 (each $1 \mathrm{H}, \mathrm{d}, J=15.8 \mathrm{~Hz}, 3$ - $O$-dimethylsuccinyl $\mathrm{H}_{2}-2$ '), $2.68(4 \mathrm{H}, \mathrm{t}, J=7.3 \mathrm{~Hz}, 28-O$ succinyl $\mathrm{H}_{2}-2 ", \mathrm{H}_{2}-3 "$ ), 3.88, 4.30 (each $1 \mathrm{H}, \mathrm{d}, J=10.8 \mathrm{~Hz}, \mathrm{H}_{2}-28$ ), 4.43 (1H, dd, $J=5.2$, $11.2 \mathrm{~Hz}, \mathrm{H}-3), 4.59,4.68$ (each 1H, br s, $\left.\mathrm{H}_{2}-29\right), 6.85\left(1 \mathrm{H}, \mathrm{s}, \mathrm{CH}(\mathrm{Ph})_{2}\right), 7.24-7.32(10 \mathrm{H}, \mathrm{m}$, aromatic-H); ${ }^{13} \mathrm{C}$ NMR $\left(\mathrm{CDCl}_{3}, 125 \mathrm{MHz}\right) \delta 14.7$ (C-27), $16.0(\mathrm{C}-26), 16.1(\mathrm{C}-25), 16.5$ (C-24), 18.1 (C-6), 19.1 (C-30), 20.7 (C-11), 23.5 (C-2), 25.1 (C-12), 25.2, 25.4 (3-Odimethylsuccinyl $\mathrm{CH}_{3}$ ), 27.0 (C-15), 27.9 (C-23), 28.8 (28-O-succinyl C-3"), 29.0 (28-Osuccinyl C-2"), 29.5 (C-21), 29.7 (C-16), 34.0 (C-7), 34.5 (C-22), 37.0 (C-10), 37.5 (C-13), 37.6 (C-4), 38.3 (C-1), 40.7 (dimethylsuccinyl C-3'), 40.8 (C-8), 42.6 (C-14), 44.5 (3-Odimethylsuccinyl C-2'), 46.4 (C-17), 47.7 (C-19), 48.7 (C-18), 50.2 (C-9), 55.3 (C-5), 63.2 (C-28), $77.0\left(\mathrm{CH}(\mathrm{Ph})_{2}\right), 81.3$ (C-3), 109.9 (C-29), 127.0 (benzhydryl C-2', 6'), 127.7 (benzyhydryl C-4'), 128.4 (benzhydryl C-3', 5'), 140.3 (benzhydryl C-1'), 150.1 (C-20), 170.9 (3-O-dimethylsuccinyl 1'-COO-), 172.5 (28-O-succinyl 1"-COO-), 175.4 (3-Odimethylsuccinyl 4'-COO-), 176.8 (28- $O$-succinyl COOH). HRESIMS (positive) $\mathrm{m} / \mathrm{z}$ $859.5112[\mathrm{M}+\mathrm{Na}]^{+}$(calcd for $\mathrm{C}_{53} \mathrm{H}_{72} \mathrm{O}_{8} \mathrm{Na}, 859.5125$ ).

3-O-(4'-Benzhydryloxy-3',3'-dimethylsuccinyl)-28-O-(3",3"'-dimethylsuccinyl)betulin (22) and 3-O-(4'-benzhydryloxy-3',3'-dimethylsuccinyl)-28-O-(2",2"- 
dimethylsuccinyl)betulin (23): Yield $68.4 \%$ and $31.6 \%$, respectively (starting from $114 \mathrm{mg}$ of $\mathbf{1 6}$ and 2,2-dimethylsuccinic acid); separated by preparative HPLC ( $\pi_{\mathrm{NAP}}, \mathrm{MeOH} / 2 \%$ HOAc $=97: 3$ ) for spectroscopic analysis. For synthesis, mixture was used for next reaction without purification.

Compound 22: $[\alpha]_{\mathrm{D}}^{26}+6.0^{\circ}\left(c 6.81, \mathrm{CHCl}_{3}\right) .{ }^{1} \mathrm{H} \mathrm{NMR}\left(\mathrm{CDCl}_{3}, 400 \mathrm{MHz}\right) \delta 0.75(3 \mathrm{H}, \mathrm{s}$, $\left.\mathrm{CH}_{3}-24\right), 0.79$ (3H, s, $\left.\mathrm{CH}_{3}-23\right), 0.80\left(3 \mathrm{H}, \mathrm{s}, \mathrm{CH}_{3}-25\right), 0.97\left(3 \mathrm{H}, \mathrm{s}, \mathrm{CH}_{3}-27\right), 1.03(3 \mathrm{H}, \mathrm{s}$, $\left.\mathrm{CH}_{3}-26\right), 1.32\left(12 \mathrm{H}\right.$, s, dimethylsuccinyl $\left.\mathrm{CH}_{3}\right), 1.70\left(3 \mathrm{H}, \mathrm{s}, \mathrm{CH}_{3}-30\right), 2.43(1 \mathrm{H}, \mathrm{dt}, J=5.6$, $10.8 \mathrm{~Hz}, \mathrm{H}-19), 2.64,2.70$ (each $1 \mathrm{H}, \mathrm{d}, J=16.0 \mathrm{~Hz}, 3-O$-dimethylsuccinyl $\mathrm{H}_{2}-2$ '), $2.66(2 \mathrm{H}$, s, 28-O-dimethylsuccinyl $\mathrm{H}_{2}-2$ "), 3.88, 4.29 (each $\left.1 \mathrm{H}, \mathrm{d}, J=10.8 \mathrm{~Hz}, \mathrm{H}_{2}-28\right), 4.45(1 \mathrm{H}$, dd, $J=5.2,10.8 \mathrm{~Hz}, \mathrm{H}-3), 4.61,4.70$ (each $1 \mathrm{H}$, br s, $\left.\mathrm{H}_{2}-29\right), 6.87\left(1 \mathrm{H}, \mathrm{s}, \mathrm{CH}(\mathrm{Ph})_{2}\right), 7.25-7.33$ $\left(10 \mathrm{H}, \mathrm{m}\right.$, aromatic-H); ${ }^{13} \mathrm{C} \mathrm{NMR}\left(\mathrm{CDCl}_{3}, 100 \mathrm{MHz}\right) \delta 14.8(\mathrm{C}-27), 16.1(\mathrm{C}-26), 16.1$ (C-25), 16.5 (C-24), 18.2 (C-6), 19.2 (C-30), 20.9 (C-11), 23.6 (C-2), 25.2 (C-12), 25.2, 25.4 (3-O-dimethylsuccinyl $\left.\mathrm{CH}_{3}\right), 25.3,25.4$ (28-O-dimethylsuccinyl $\left.\mathrm{CH}_{3}\right), 27.0(\mathrm{C}-15), 27.9$ (C-23), 29.6 (C-21), 29.8 (C-16), 34.2 (C-7), 34.6 (C-22), 37.1 (C-10), 37.7 (C-4, C-13), 38.4 (C-1), 40.7 (dimethylsuccinyl C-3', C-3"), 40.9 (C-8), 42.7 (C-14), 44.4 (28-Odimethylsuccinyl C-2"), 44.6 (3-O-dimethylsuccinyl C-2'), 46.3 (C-17), 47.7 (C-19), 48.9 (C-18), 50.3 (C-9), 55.4 (C-5), 63.1 (C-28), $77.0\left(\mathrm{CH}(\mathrm{Ph})_{2}\right), 81.3$ (C-3), 109.8 (C-29), 127.0, 127.1 (benzhydryl C-2', 6'), 127.7 (benzyhydryl C-4'), 128.4 (benzhydryl C-3', 5'), 140.4 (benzhydryl C-1'), 150.1 (C-20), 170.8 (3-O-dimethylsuccinyl 1'-COO-), 171.5 (28-Odimethylsuccinyl 1"-COO-), 175.4 (3- $O$-dimethylsuccinyl 4'-COO-), 182.4 (28-Odimethylsuccinyl $\mathrm{COOH}$ ). HRESIMS (positive) $\mathrm{m} / z 887.5421[\mathrm{M}+\mathrm{Na}]^{+}$(calcd for $\left.\mathrm{C}_{55} \mathrm{H}_{76} \mathrm{O}_{8} \mathrm{Na}, 887.5438\right)$.

Compound 23: $[\alpha]_{\mathrm{p}}^{27}+6.3^{\circ}\left(c 3.15, \mathrm{CHCl}_{3}\right) .{ }^{1} \mathrm{H} \mathrm{NMR}\left(\mathrm{CDCl}_{3}, 400 \mathrm{MHz}\right) \delta 0.75(3 \mathrm{H}, \mathrm{s}$, $\left.\mathrm{CH}_{3}-24\right), 0.79$ (3H, s, $\left.\mathrm{CH}_{3}-23\right), 0.80\left(3 \mathrm{H}, \mathrm{s}, \mathrm{CH}_{3}-25\right), 0.98\left(3 \mathrm{H}, \mathrm{s}, \mathrm{CH}_{3}-27\right), 1.03(3 \mathrm{H}, \mathrm{s}$, $\left.\mathrm{CH}_{3}-26\right), 1.31,1.32$ (each $3 \mathrm{H}, \mathrm{s}, 3-O$-dimethylsuccinyl $\left.\mathrm{CH}_{3}\right), 1.32(6 \mathrm{H}, \mathrm{s}, 28-O$ dimethylsuccinyl $\left.\mathrm{CH}_{3}\right), 1.70\left(3 \mathrm{H}, \mathrm{s}, \mathrm{CH}_{3}-30\right), 2.44(1 \mathrm{H}, \mathrm{dt}, J=5.6,10.8 \mathrm{~Hz}, \mathrm{H}-19), 2.64$, 2.70 (each $1 \mathrm{H}, \mathrm{d}, J=15.6 \mathrm{~Hz}, 3$ - $O$-dimethylsuccinyl $\mathrm{H}_{2}-2$ '), $2.66(2 \mathrm{H}, \mathrm{s}, 28-O$ dimethylsuccinyl $\mathrm{H}_{2}-3$ "), 3.85, 4.32 (each $1 \mathrm{H}, \mathrm{d}, J=10.8 \mathrm{~Hz}, \mathrm{H}_{2}-28$ ), 4.45 (1H, dd, $J=5.2$, $10.8 \mathrm{~Hz}, \mathrm{H}-3$ ), 4.61, 4.70 (each 1H, br s, $\left.\mathrm{H}_{2}-29\right), 6.87\left(1 \mathrm{H}, \mathrm{s}, \mathrm{CH}(\mathrm{Ph})_{2}\right), 7.24-7.32(10 \mathrm{H}, \mathrm{m}$, aromatic-H); ${ }^{13} \mathrm{C}$ NMR $\left(\mathrm{CDCl}_{3}, 100 \mathrm{MHz}\right) \delta 14.8(\mathrm{C}-27), 16.0(\mathrm{C}-26), 16.1(\mathrm{C}-25), 16.5$ (C-24), 18.2 (C-6), 19.2 (C-30), 20.8 (C-11), 23.6 (C-2), 25.2 (C-12), 25.2, 25.4 (3-Odimethylsuccinyl $\left.\mathrm{CH}_{3}\right), 25.3,25.5$ (28-O-dimethylsuccinyl $\left.\mathrm{CH}_{3}\right), 27.0(\mathrm{C}-15), 27.9(\mathrm{C}-23)$, 29.6 (C-21), 29.9 (C-16), 34.1 (C-7), 34.5 (C-22), 37.1 (C-10), 37.7 (C-4, 13), 38.4 (C-1), 40.7 (dimethylsuccinyl C-3', C-2"), 40.9 (C-8), 42.7 (C-14), 44.0 (28-O-dimethylsuccinyl C-3"), 44.6 (3-O-dimethylsuccinyl C-2'), 46.6 (C-17), 47.8 (C-19), 48.9 (C-18), 50.3 (C-9), 55.4 (C-5), $63.3(\mathrm{C}-28), 77.0\left(\mathrm{CH}(\mathrm{Ph})_{2}\right), 81.3$ (C-3), 109.8 (C-29), 127.0, 127.1 (benzhydryl C-2', 6'), 127.7 (benzyhydryl C-4'), 128.4 (benzhydryl C-3', 5'), 140.4 (benzhydryl C-1'), 150.1 (C-20), 170.8 (3-O-dimethylsuccinyl 1'-COO-), 175.4 (3-O-dimethylsuccinyl 4'-COO -, 28-O-dimethylsuccinyl $\mathrm{COOH}), 177.0$ (28-O-dimethylsuccinyl 1"-COO-). HRESIMS (positive) $\mathrm{m} / z 887.5440[\mathrm{M}+\mathrm{Na}]^{+}\left(\right.$calcd for $\mathrm{C}_{55} \mathrm{H}_{76} \mathrm{O}_{8} \mathrm{Na}, 887.5438$ ).

\section{3-O-(4'-Benzhydryloxy-3',3'-dimethylsuccinyl)-28-O-glutarylbetulin (24): Yield 59.5\%}

(starting from $406 \mathrm{mg}$ of $\mathbf{1 6}$ and glutaric anhydride); white solid. $[\alpha]_{\mathrm{D}}^{15}+10.8^{\circ}(c 0.5$, $\left.\mathrm{CHCl}_{3}\right) .{ }^{1} \mathrm{H} \mathrm{NMR}\left(\mathrm{CDCl}_{3}, 500 \mathrm{MHz}\right) \delta 0.73\left(3 \mathrm{H}, \mathrm{s}, \mathrm{CH}_{3}-24\right), 0.77\left(6 \mathrm{H}, \mathrm{s}, \mathrm{CH}_{3}-23\right.$ and $\left.\mathrm{CH}_{3}-25\right), 0.96\left(3 \mathrm{H}, \mathrm{s}, \mathrm{CH}_{3}-27\right), 1.01\left(3 \mathrm{H}, \mathrm{s}, \mathrm{CH}_{3}-26\right), 1.30,1.31$ (each $3 \mathrm{H}, \mathrm{s}$, dimethylsuccinyl $\left.\mathrm{CH}_{3}\right), 1.68\left(3 \mathrm{H}, \mathrm{s}, \mathrm{CH}_{3}-30\right), 1.97(2 \mathrm{H}$, quint, $J=7.2 \mathrm{~Hz}, 28$ - $O$-glutaryl $\mathrm{H}_{2}-3$ "), 2.41-2.46 (1H, m, $\left.J=5.5,10.9 \mathrm{~Hz}, \mathrm{H}-19\right), 2.43(2 \mathrm{H}, \mathrm{t}, J=7.2 \mathrm{~Hz}, 28-O$-glutaryl $\mathrm{H}_{2}-2$ "), $2.44\left(2 \mathrm{H}, \mathrm{t}, J=7.2 \mathrm{~Hz}, 28\right.$ - $O$-glutaryl $\mathrm{H}_{2}-4 "$ ), 2.64, 2.69 (each $1 \mathrm{H}, \mathrm{d}, J=15.8 \mathrm{~Hz}, 3-$ $O$-dimethylsuccinyl $\mathrm{H}_{2}-2$ '), 3.85, 4.28 (each $\left.1 \mathrm{H}, \mathrm{d}, J=10.8 \mathrm{~Hz}, \mathrm{H}_{2}-28\right), 4.43(1 \mathrm{H}, \mathrm{dd}, J=$ 
5.2, 11.4 Hz, H-3), 4.59, 4.68 (each $1 \mathrm{H}$, br s, $\left.\mathrm{H}_{2}-29\right), 6.85\left(1 \mathrm{H}, \mathrm{s}, \mathrm{CH}(\mathrm{Ph})_{2}\right), 7.24-7.32$ $\left(10 \mathrm{H}, \mathrm{m}\right.$, aromatic-H); ${ }^{13} \mathrm{C} \mathrm{NMR}\left(\mathrm{CDCl}_{3}, 125 \mathrm{MHz}\right) \delta 14.7(\mathrm{C}-27), 16.0(\mathrm{C}-26), 16.1$ (C-25), 16.5 (C-24), 18.1 (C-6), 19.1 (C-30), 20.0 (28-O-glutaryl C-3"), 20.8 (C-11), 23.5 (C-2), 25.1 (C-12), 25.2, 25.4 (3-O-dimethylsuccinyl $\left.\mathrm{CH}_{3}\right), 27.0$ (C-15), 27.9 (C-23), 29.5 (C-21), 29.7 (C-16), 32.8 (28-O-glutaryl C-4"), 33.3 (28-O-glutaryl C-2"), 34.1 (C-7), 34.5 (C-22), 37.0 (C-10), 37.6 (2C, C-13 and C-4), 38.4 (C-1), 40.7 (3-O-dimethylsuccinyl C-3'), 40.9 (C-8), 42.7 (C-14), 44.5 (3-O-dimethylsuccinyl C-2'), 46.4 (C-17), 47.7 (C-19), 48.8 (C-18), 50.2 (C-9), 55.3 (C-5), 62.8 (C-28), $77.0\left(\mathrm{CH}(\mathrm{Ph})_{2}\right), 81.3$ (C-3), 109.9 (C-29), 127.0 (benzhydryl C-2', 6'), 127.7 (benzyhydryl C-4'), 128.4 (benzhydryl C-3', 5'), 140.3 (benzhydryl C-1'), 150.1 (C-20), 170.9 (3-O-dimethylsuccinyl 1'-COO-), 173.3 (28-Oglutaryl 1"-COO-), 175.4 (3-O-dimethylsuccinyl 4'-COO-), 177.7 (28-O-glutaryl $\mathrm{COOH})$. HRESIMS (positive) $\mathrm{m} / z$ 873.5286 [M+Na] ${ }^{+}$(calcd for $\mathrm{C}_{54} \mathrm{H}_{74} \mathrm{O}_{8} \mathrm{Na}, 873.5281$ ).

3-O-(4'-Benzhydryloxy-3',3'-dimethylsuccinyl)-28-O-(3",3"-dimethylglutaryl)betulin (25): Yield $87.0 \%$ (starting from $222 \mathrm{mg}$ of $\mathbf{1 6}$ and 3,3-dimethylglutaric acid); white solid. $[\alpha]_{\mathrm{D}}^{25}+6.8^{\circ}\left(c 3.59, \mathrm{CHCl}_{3}\right) .{ }^{1} \mathrm{H} \mathrm{NMR}\left(\mathrm{CDCl}_{3}, 400 \mathrm{MHz}\right) \delta 0.74\left(3 \mathrm{H}, \mathrm{s}, \mathrm{CH}_{3}-24\right), 0.79(3 \mathrm{H}$, $\left.\mathrm{s}, \mathrm{CH}_{3}-23\right), 0.79\left(3 \mathrm{H}, \mathrm{s}, \mathrm{CH}_{3}-25\right), 0.98\left(3 \mathrm{H}, \mathrm{s}, \mathrm{CH}_{3}-27\right), 1.03\left(3 \mathrm{H}, \mathrm{s}, \mathrm{CH}_{3}-26\right), 1.16(6 \mathrm{H}, \mathrm{s}$, dimethylglutaryl $\left.\mathrm{CH}_{3}\right), 1.31,1.32$ (each $3 \mathrm{H}$, s, dimethylsuccinyl $\left.\mathrm{CH}_{3}\right), 1.70(3 \mathrm{H}, \mathrm{s}$, $\mathrm{CH}_{3}-30$ ), $2.40-2.50$ (1H, m, H-19), 2.47, 2.48 (each $2 \mathrm{H}, \mathrm{s}$, dimethylglutaryl $\mathrm{H}_{2}-2 ", 4 "$ ), 2.65, 2.70 (each $1 \mathrm{H}, \mathrm{d}, J=16.0 \mathrm{~Hz}, 3-O$-dimethylsuccinyl $\mathrm{H}_{2}-2$ '), 3.86, 4.29 (each $1 \mathrm{H}, \mathrm{d}, J=$ $\left.10.8 \mathrm{~Hz}, \mathrm{H}_{2}-28\right), 4.44(1 \mathrm{H}, \mathrm{dd}, J=5.2,10.8 \mathrm{~Hz}, \mathrm{H}-3), 4.60,4.70$ (each $1 \mathrm{H}$, br s, $\mathrm{H}_{2}-29$ ), $6.87\left(1 \mathrm{H}, \mathrm{s}, \mathrm{CH}(\mathrm{Ph})_{2}\right), 7.24-7.33(10 \mathrm{H}, \mathrm{m}$, aromatic-H $) ;{ }^{13} \mathrm{C} \mathrm{NMR}\left(\mathrm{CDCl}_{3}, 100 \mathrm{MHz}\right) \delta$ 14.7 (C-27), 16.0 (C-26), 16.1 (C-25), 16.5 (C-24), 18.1 (C-6), 19.1 (C-30), 20.8 (C-11), 23.5 (C-2), 25.2 (C-12), 25.2, 25.4 (3-O-dimethylsuccinyl $\mathrm{CH}_{3}$ ), 26.9 (C-15), 27.8 (28-Odimethylsuccinyl $\mathrm{CH}_{3}$ ), 27.9 (C-23), 29.5 (C-21), 29.8 (C-16), (dimethylglutaryl C-3"), 34.0 (C-7), 34.6 (C-22), 37.0 (C-10), 37.6 (C-4, 13), 38.3 (C-1), (dimethylsuccinyl C-3'), 40.8 (C-8), 42.6 (C-14), 44.5 (3-O-dimethylsuccinyl C-2'), 45.1 (dimethylglutaryl C-4"), 45.3 (dimethylglutaryl C-2"), 46.2 (C-17), 47.7 (C-19), 48.8 (C-18), 50.2 (C-9), 55.3 (C-5), 62.8 (C-28), $77.0\left(\mathrm{CH}(\mathrm{Ph})_{2}\right), 81.3(\mathrm{C}-3), 109.9$ (C-29), 127.0 (benzhydryl C-2', 6'), 127.7 (benzyhydryl C-4'), 128.4 (benzhydryl C-3', 5'), 140.3 (benzhydryl C-1'), 150.0 (C-20), 170.9 (3-O-dimethylsuccinyl 1'-COO-), 172.7 (28-O-dimethylglutaryl 1"-COO-), 175.4 (3$O$-dimethylsuccinyl 4'-COO-), 176.9 (28- $O$-dimethylglutaryl COOH). HRESIMS (positive) $\mathrm{m} / z, 901.5583[\mathrm{M}+\mathrm{Na}]^{+}$(calcd for $\mathrm{C}_{56} \mathrm{H}_{78} \mathrm{O}_{8} \mathrm{Na}, 901.5594$ ).

\section{3-O-(5'-Benzhydryloxy-3',3'-dimethylglutaryl)-28-O-(3",3"-dimethylglutaryl)betulin} (26): Yield 100\% (starting from $150 \mathrm{mg}$ of $\mathbf{1 7}$ and 3,3-dimethylglutaric anhydride); white solid. $[\alpha]_{\mathrm{D}}^{16}+7.3^{\circ}\left(c 3.36, \mathrm{CHCl}_{3}\right) .{ }^{1} \mathrm{H} \mathrm{NMR}\left(\mathrm{CDCl}_{3}, 400 \mathrm{MHz}\right) \delta 0.81\left(3 \mathrm{H}, \mathrm{s}, \mathrm{CH}_{3}-24\right), 0.84$ $\left(3 \mathrm{H}, \mathrm{s}, \mathrm{CH}_{3}-23,25\right), 0.98\left(3 \mathrm{H}, \mathrm{s}, \mathrm{CH}_{3}-27\right), 1.04\left(3 \mathrm{H}, \mathrm{s}, \mathrm{CH}_{3}-26\right), 1.09$ (6H, s, 3-Odimethylglutaryl $\left.\mathrm{CH}_{3}\right), 1.16\left(6 \mathrm{H}, \mathrm{s}, 28-O\right.$-dimethylglutaryl $\left.\mathrm{CH}_{3}\right), 1.70\left(3 \mathrm{H}, \mathrm{s}, \mathrm{CH}_{3}-30\right)$, 2.43-2.45 (1H, m, H-19), 2.36, 2.43 (each $1 \mathrm{H}, \mathrm{d}, J=14.4 \mathrm{~Hz}, 3$ - $O$-dimethylglutaryl $\mathrm{H}_{2}-2$ '), 2.47, 2.48 (each $2 \mathrm{H}, \mathrm{s}, 28$ - $O$-dimethylglutaryl $\mathrm{H}_{2}-2 ", 4 "$ ), 2.55, 2.58 (each $1 \mathrm{H}, \mathrm{d}, J=14.4$ $\mathrm{Hz}, 3$ - $O$-dimethylglutaryl $\mathrm{H}_{2}-4^{\prime}$ ), 3.86, 4.29 (each $\left.1 \mathrm{H}, \mathrm{d}, J=11.2 \mathrm{~Hz}, \mathrm{H}_{2}-28\right), 4.47(1 \mathrm{H}$, dd, $J=4.8,11.2 \mathrm{~Hz}, \mathrm{H}-3), 4.60,4.70$ (each $1 \mathrm{H}$, br s, $\left.\mathrm{H}_{2}-29\right), 6.89\left(1 \mathrm{H}, \mathrm{s}, \mathrm{CH}(\mathrm{Ph})_{2}\right), 7.25-7.33$ $\left(10 \mathrm{H}, \mathrm{m}\right.$, aromatic-H); ${ }^{13} \mathrm{C} \mathrm{NMR}\left(\mathrm{CDCl}_{3}, 100 \mathrm{MHz}\right) \delta 14.7(\mathrm{C}-27), 16.0(\mathrm{C}-26), 16.1$ (C-25), 16.6 (C-24), 18.1 (C-6), 19.1 (C-30), 20.8 (C-11), 23.8 (C-2), 25.2 (C-12), 27.0 (C-15), 27.6, 27.7 (3-O-dimethylglutaryl $\mathrm{CH}_{3}$ ), 27.8 (28-O-dimethylglutaryl $\left.\mathrm{CH}_{3}\right), 28.0$ (C-23), 29.5 (C-21), 29.8 (C-16), 32.6 (28-O-dimethylglutaryl C-3"), 32.8 (28-Odimethylglutaryl C-3'), 34.1 (C-7), 34.6 (C-22), 37.0 (C-10), 37.6 (C-4, 13), 38.4 (C-1), 40.9 (C-8), 42.7 (C-14), 45.1 (28-O-dimethylglutaryl C-4"), 45.3 (28-O-dimethylglutaryl C-2"), 45.4 (3- $O$-dimethylglutaryl C-4'), 45.8 (3-O-dimethylglutaryl C-2'), 46.2 (C-17), 47.7 (C-19), 48.8 (C-18), 50.2 (C-9), 55.4 (C-5), 62.9 (C-28), 76.7 (CH(Ph) 2 ), 80.9 (C-3), 109.9 (C-29), 127.1 (benzhydryl C-2', 6'), 127.8 (benzyhydryl C-4'), 128.4 (benzhydryl C-3', 5'), 
140.2, 140.3 (benzhydryl C-1'), 150.0 (C-20), 170.8 (3-O-dimethylglutaryl 5'-COO-), 171.7 (3-O-dimethylglutaryl 1'-COO-), 172.8 (28-O-dimethylglutaryl 1"-COO-), 176.5 (28-Odimethylglutaryl $\mathrm{COOH}$ ). HRESIMS (positive) $\mathrm{m} / z 915.5771[\mathrm{M}+\mathrm{Na}]^{+}$(calcd for $\mathrm{C}_{57} \mathrm{H}_{80} \mathrm{O}_{8} \mathrm{Na}, 915.5751$ ).

3-O-(4'-Benzhydryloxy-3',3'-dimethylsuccinyl)-28-O-(3",3"-dimethylsuccinyl)dihydrobetulin (27) and 3-O-(4'-benzhydryloxy-3',3'dimethylsuccinyl)-28-O-(2",2"-dimethylsuccinyl)dihydrobetulin (28): Total yield 96.3\% (starting from $330 \mathrm{mg}$ of $\mathbf{1 8}$ and 2,2-dimethylsuccinic acid); used in next reaction without purification. For spectroscopic analysis, a small amount of sample was further purified by preparative HPLC $\left(\pi_{\mathrm{NAP}}, \mathrm{MeOH} / 2 \% \mathrm{HOAc}=97: 3\right)$; white solid.

Compound 27: $[\alpha]_{\mathrm{D}}^{26}-12.3^{\circ}\left(c 3.50, \mathrm{CHCl}_{3}\right) .{ }^{1} \mathrm{H} \mathrm{NMR}\left(\mathrm{CDCl}_{3}, 400 \mathrm{MHz}\right) \delta 0.75(3 \mathrm{H}, \mathrm{s}$, $\left.\mathrm{CH}_{3}-24\right), 0.77,0.85$ (each $\left.3 \mathrm{H}, \mathrm{d}, J=8.0 \mathrm{~Hz}, \mathrm{CH}_{3}-29,30\right), 0.79\left(3 \mathrm{H}, \mathrm{s}, \mathrm{CH}_{3}-23\right), 0.80$ (3H, s, $\left.\mathrm{CH}_{3}-25\right), 0.94\left(3 \mathrm{H}, \mathrm{s}, \mathrm{CH}_{3}-27\right), 1.03\left(3 \mathrm{H}, \mathrm{s}, \mathrm{CH}_{3}-26\right), 1.31,1.32$ (each $3 \mathrm{H}, \mathrm{s}, 3-O-$ dimethylsuccinyl $\left.\mathrm{CH}_{3}\right), 1.31\left(6 \mathrm{H}, \mathrm{s}, 28-O\right.$-dimethylsuccinyl $\left.\mathrm{CH}_{3}\right), 2.65(2 \mathrm{H}, \mathrm{s}, 28-O$ dimethylsuccinyl $\mathrm{H}_{2}-2$ "), 2.66, 2.70 (each $1 \mathrm{H}, \mathrm{d}, J=15.6 \mathrm{~Hz}, 3-O$-dimethylsuccinyl $\mathrm{H}_{2}-2^{\prime}$ ), 3.85, 4.26 (each 1H, d, $\left.J=11.2 \mathrm{~Hz}, \mathrm{H}_{2}-28\right), 4.45(1 \mathrm{H}, \mathrm{dd}, J=5.2,10.8 \mathrm{~Hz}, \mathrm{H}-3), 6.87(1 \mathrm{H}$, s, $\left.\mathrm{CH}(\mathrm{Ph})_{2}\right), 7.25-7.33\left(10 \mathrm{H}, \mathrm{m}\right.$, aromatic-H); ${ }^{13} \mathrm{C} \mathrm{NMR}\left(\mathrm{CDCl}_{3}, 100 \mathrm{MHz}\right) \delta 14.6(\mathrm{C}-27)$, 14.9 (C-29), 16.0 (C-25, C-26), 16.5 (C-24), 18.1 (C-6), 20.8 (C-11), 21.5 (C-21), 22.9 (C-30), 23.5 (C-2), 25.2, 25.4 (3-O-dimethylsuccinyl $\left.\mathrm{CH}_{3}\right), 25.3,25.4$ (28-Odimethylsuccinyl $\left.\mathrm{CH}_{3}\right), 26.8$ (C-12, C-15), 27.9 (C-23), 29.4 (C-20), 29.8 (C-16), 34.1 (C-7), 34.6 (C-22), 36.9 (C-10), 37.1 (C-13), 37.6 (C-4), 38.3 (C-1), 40.5 (28-Odimethylsuccinyl C-3"), 40.7 (3-O-dimethylsuccinyl C-3'), 40.9 (C-8), 42.8 (C-14), 44.4 (28-O-dimethylsuccinyl C-2"), 44.5 (C-19, 3-O-dimethylsuccinyl C-2'), 46.4 (C-17), 48.1 (C-18), 49.9 (C-9), 55.3 (C-5), 63.1 (C-28), $77.0\left(\mathrm{CH}(\mathrm{Ph})_{2}\right), 81.3(\mathrm{C}-3), 127.0,127.1$ (benzhydryl C-2', 6'), 127.7 (benzyhydryl C-4'), 128.4 (benzhydryl C-3', 5'), 140.3 (benzhydryl C-1'), 170.9 (3-O-dimethylsuccinyl $1^{\prime}-\mathrm{COO}-$ ), 171.6 (28- $O$-dimethylsuccinyl 1"-COO-), 175.4 (3-O-dimethylsuccinyl 4'-COO-), 182.9 (28-O-dimethylsuccinyl $\mathrm{COOH}$ ). HRESIMS (positive) $\mathrm{m} / z 889.5585[\mathrm{M}+\mathrm{Na}]^{+}$(calcd for $\mathrm{C}_{55} \mathrm{H}_{78} \mathrm{O}_{8} \mathrm{Na}, 889.5594$ ).

Compound 28: $[\alpha]_{\mathrm{D}}^{27}-11.4^{\circ}\left(c 2.30, \mathrm{CHCl}_{3}\right) .{ }^{1} \mathrm{H} \mathrm{NMR}\left(\mathrm{CDCl}_{3}, 400 \mathrm{MHz}\right) \delta 0.75(3 \mathrm{H}, \mathrm{s}$, $\left.\mathrm{CH}_{3}-24\right), 0.78,0.85$ (each $\left.3 \mathrm{H}, \mathrm{d}, J=8.0 \mathrm{~Hz}, \mathrm{CH}_{3}-29,30\right), 0.79$ (3H, s, $\left.\mathrm{CH}_{3}-23\right), 0.80$ (3H, s, $\left.\mathrm{CH}_{3}-25\right), 0.95\left(3 \mathrm{H}, \mathrm{s}, \mathrm{CH}_{3}-27\right), 1.03\left(3 \mathrm{H}, \mathrm{s}, \mathrm{CH}_{3}-26\right), 1.32,1.31$ (each $3 \mathrm{H}, \mathrm{s}, 3-\mathrm{O}$ dimethylsuccinyl $\mathrm{CH}_{3}$ ), 1.31 (each $3 \mathrm{H}, \mathrm{s}, 28-O$-dimethylsuccinyl $\mathrm{CH}_{3}$ ), 2.66, 2.70 (each $1 \mathrm{H}$, d, $J=15.6 \mathrm{~Hz}, 3-O$-dimethylsuccinyl $\left.\mathrm{H}_{2}-2^{\prime}\right), 2.65\left(2 \mathrm{H}, \mathrm{s}, 28\right.$ - $O$-dimethylsuccinyl $\left.\mathrm{H}_{2}-3 "\right)$, 3.79, 4.31 (each 1H, d, $\left.J=10.8 \mathrm{~Hz}, \mathrm{H}_{2}-28\right), 4.45(1 \mathrm{H}, \mathrm{dd}, J=5.2,11.2 \mathrm{~Hz}, \mathrm{H}-3), 6.87(1 \mathrm{H}$, $\left.\mathrm{s}, \mathrm{CH}(\mathrm{Ph})_{2}\right), 7.25-7.33\left(10 \mathrm{H}, \mathrm{m}\right.$, aromatic-H); ${ }^{13} \mathrm{C} \mathrm{NMR}\left(\mathrm{CDCl}_{3}, 100 \mathrm{MHz}\right) \delta 14.6(\mathrm{C}-27)$, 14.9 (C-29), 16.0 (C-25, C-26), 16.5 (C-24), 18.1 (C-6), 20.8 (C-11), 21.5 (C-21), 22.9 (C-30), 23.5 (C-2), 25.2, 25.4 (3-O-dimethylsuccinyl $\mathrm{CH}_{3}$ ), 25.4, 25.5 (28-Odimethylsuccinyl $\mathrm{CH}_{3}$ ), 26.8 (C-12, C-15), 27.9 (C-23), 29.4 (C-20), 29.9 (C-16), 34.1 (C-7), 34.7 (C-22), 37.0 (C-10), 37.1 (C-13), 37.7 (C-4), 38.4 (C-1), 40.7 (3-Odimethylsuccinyl C-3', 28-O-dimethylsuccinyl 2"), 40.9 (C-8), 42.8 (C-14), 43.9 (28-Odimethylsuccinyl C-3"), 44.5 (C-19, 3-O-dimethylsuccinyl C-2'), 46.6 (C-17), 48.2 (C-18), 49.9 (C-9), 55.3 (C-5), $63.3(\mathrm{C}-28), 77.0\left(\mathrm{CH}(\mathrm{Ph})_{2}\right), 81.3(\mathrm{C}-3), 127.0,127.1$ (benzhydryl C-2', 6'), 127.7 (benzyhydryl C-4'), 128.4 (benzhydryl C-3', 5'), 140.3 (benzhydryl C-1'), 170.9 (3-O-dimethylsuccinyl 1'-COO-), 175.4 (3-O-dimethylsuccinyl 4'-COO-, 28-Odimethylsuccinyl 1"-COOH), 177.0 (28-O-dimethylsuccinyl 1"-COO-). HRESIMS (positive) $\mathrm{m} / z 889.5590[\mathrm{M}+\mathrm{Na}]^{+}$(calcd for $\mathrm{C}_{55} \mathrm{H}_{78} \mathrm{O}_{8} \mathrm{Na}, 889.5594$ ).

3-O-(4'-Benzhydryloxy-3',3'-dimethylsuccinyl)-28-O-(3",3"-dimethylglutaryl)dihydrobetulin (29): Yield 100\% (starting from $110 \mathrm{mg}$ of $\mathbf{1 8}$ and 3,3- 
dimethylglutaric anhydride); white solid. $[\alpha]_{\mathrm{D}}^{26}-10.2^{\circ}\left(c 2.04, \mathrm{CHCl}_{3}\right) .{ }^{1} \mathrm{H} \mathrm{NMR}\left(\mathrm{CDCl}_{3}\right.$, $400 \mathrm{MHz}) \delta 0.74\left(3 \mathrm{H}, \mathrm{s}, \mathrm{CH}_{3}-24\right), 0.78\left(3 \mathrm{H}, \mathrm{s}, \mathrm{CH}_{3}-23\right), 0.78,0.85$ (each $3 \mathrm{H}, \mathrm{d}, J=8.0 \mathrm{~Hz}$, $\left.\mathrm{CH}_{3}-29,30\right), 0.80\left(3 \mathrm{H}, \mathrm{s}, \mathrm{CH}_{3}-25\right), 0.95\left(3 \mathrm{H}, \mathrm{s}, \mathrm{CH}_{3}-27\right), 1.04\left(3 \mathrm{H}, \mathrm{s}, \mathrm{CH}_{3}-26\right), 1.15(6 \mathrm{H}, \mathrm{s}$, dimethylglutaryl $\mathrm{CH}_{3}$ ), 1.31, 1.32 (each $3 \mathrm{H}$, s, dimethylsuccinyl $\mathrm{CH}_{3}$ ), 2.46, 2.48 (each $2 \mathrm{H}$, s, dimethylglutaryl $\mathrm{H}_{2}-2 "$ ", 4"), 2.65, 2.71 (each $1 \mathrm{H}, \mathrm{d}, J=16.0 \mathrm{~Hz}, 3$-O-dimethylsuccinyl $\left.\mathrm{H}_{2}-2^{\prime}\right), 3.83,4.28$ (each $\left.1 \mathrm{H}, \mathrm{d}, J=10.8 \mathrm{~Hz}, \mathrm{H}_{2}-28\right), 4.45(1 \mathrm{H}, \mathrm{dd}, J=5.2,11.2 \mathrm{~Hz}, \mathrm{H}-3$ ), $6.86\left(1 \mathrm{H}, \mathrm{s}, \mathrm{CH}(\mathrm{Ph})_{2}\right), 7.25-7.33\left(10 \mathrm{H}, \mathrm{m}\right.$, aromatic-H); ${ }^{13} \mathrm{C} \mathrm{NMR}\left(\mathrm{CDCl}_{3}, 100 \mathrm{MHz}\right) \delta$ 14.6 (C-27), 14.9 (C-29), 16.0 (C-25, C-26), 16.5 (C-24), 18.1 (C-6), 20.7(C-11), 21.6 (C-21), 22.9 (C-30), 23.5 (C-2), 25.2, 25.4 (3-O-dimethylsuccinyl $\left.\mathrm{CH}_{3}\right), 26.8$ (C-12), 26.9 (C-15), 27.9 (C-23, 28-O-dimethylsuccinyl $\left.\mathrm{CH}_{3}\right), 29.4(\mathrm{C}-20), 29.9(\mathrm{C}-16), 32.7$ (dimethylglutaryl C-3"), 34.1 (C-7), 34.7 (C-22), 37.0 (C-10), 37.2 (C-13), 37.6 (C-4), 38.4 (C-1), 40.7 (dimethylsuccinyl C-3'), 40.9 (C-8), 42.8 (C-14), 44.5 (C-19, 3-O-

dimethylsuccinyl C-2'), 45.1 (dimethylglutaryl C-4"), 45.4 (dimethylglutaryl C-2"), 46.4 (C-17), 48.1 (C-18), 49.9 (C-9), 55.3 (C-5), 63.0 (C-28), $77.0\left(\mathrm{CH}(\mathrm{Ph})_{2}\right), 81.3$ (C-3), 127.0 (benzhydryl C-2', 6'), 127.7 (benzyhydryl C-4'), 128.4 (benzhydryl C-3', 5'), 140.3 (benzhydryl C-1'), 170.9 (3-O-dimethylsuccinyl 1'-COO-), 173.0 (28-O-dimethylglutaryl 1"-COO-), 175.4 (3-O-dimethylsuccinyl 4'-COO-), 176.0 (28-O-dimethylglutaryl $\mathrm{COOH}$ ). HRESIMS (positive) $\mathrm{m} / z$ $903.5748[\mathrm{M}+\mathrm{Na}]^{+}$(calcd for $\mathrm{C}_{56} \mathrm{H}_{80} \mathrm{O}_{8} \mathrm{Na}, 903.5751$ ).

3-O-(5'-Benzhydryloxyglutaryl)-28-O-(3",3"-dimethylsuccinyl)dihydrobetulin (30) and 3-O-(5'-benzhydryloxyglutaryl)-28- $O$ - $\left(2^{\prime \prime}, 2^{\prime \prime}\right.$-dimethylsuccinyl)dihydrobetulin (31): Total yield $98.9 \%$ (starting from $225 \mathrm{mg}$ of $\mathbf{2 0}$ and 2,2-dimethylsuccinic acid); used for next reaction without purification. For spectroscopic analysis, a small amount of sample was further purified by preparative $\mathrm{HPLC}\left(\pi_{\mathrm{NAP}}, \mathrm{CH}_{3} \mathrm{OH} / \mathrm{H}_{2} \mathrm{O} / \mathrm{CH}_{3} \mathrm{COOH}=95: 4: 1\right)$; colorless oil.

Compound 30: $[\alpha]_{\mathrm{D}}^{27}-7.1^{\circ}\left(\right.$ c 3.50, $\left.\mathrm{CHCl}_{3}\right) .{ }^{1} \mathrm{H} \mathrm{NMR}\left(\mathrm{CDCl}_{3}, 400 \mathrm{MHz}\right) \delta 0.78,0.85$ (each $\left.3 \mathrm{H}, \mathrm{d}, J=8.0 \mathrm{~Hz}, \mathrm{CH}_{3}-29,30\right), 0.84$ (each $\left.3 \mathrm{H}, \mathrm{s}, \mathrm{CH}_{3}-23,24\right), 0.87$ (3H, s, $\left.\mathrm{CH}_{3}-25\right), 0.96$ $\left(3 \mathrm{H}, \mathrm{s}, \mathrm{CH}_{3}-27\right), 1.05\left(3 \mathrm{H}, \mathrm{s}, \mathrm{CH}_{3}-26\right), 1.32\left(6 \mathrm{H}, \mathrm{s}, 28-O\right.$-dimethylsuccinyl $\left.\mathrm{CH}_{3}\right), 2.00(2 \mathrm{H}$, quint, $J=7.2 \mathrm{~Hz}, 3$ - $O$-glutaryl $\left.\mathrm{H}_{2}-3^{\prime}\right), 2.36\left(2 \mathrm{H}, \mathrm{t}, J=7.2 \mathrm{~Hz}, 3\right.$ - $O$-glutaryl $\left.\mathrm{H}_{2}-2^{\prime}\right), 2.51(2 \mathrm{H}$, $\mathrm{t}, J=7.2 \mathrm{~Hz}, 3$ - $O$-glutaryl $\mathrm{H}_{2}-4$ '), $2.65\left(2 \mathrm{H}, \mathrm{s}, 28\right.$ - $O$-dimethylsuccinyl $\left.\mathrm{H}_{2}-2 "\right), 3.85,4.28$ (each $\left.1 \mathrm{H}, \mathrm{d}, J=10.8 \mathrm{~Hz}, \mathrm{H}_{2}-28\right), 4.49(1 \mathrm{H}, \mathrm{dd}, J=6.0,10.0 \mathrm{~Hz}, \mathrm{H}-3), 6.90(1 \mathrm{H}, \mathrm{s}$, $\left.\mathrm{CH}(\mathrm{Ph})_{2}\right), 7.26-7.34(10 \mathrm{H}, \mathrm{m}$, aromatic-H $) ;{ }^{13} \mathrm{C} \mathrm{NMR}\left(\mathrm{CDCl}_{3}, 100 \mathrm{MHz}\right) \delta 14.6(\mathrm{C}-27)$, 14.9 (C-29), 16.1 (C-25, C-26), 16.5 (C-24), 18.2 (C-6), 20.3 (glutaryl C-3'), 20.8 (C-11), 21.6 (C-21), 22.9 (C-30), 23.7 (C-2), 25.3 (28-O-dimethylsuccinyl $\mathrm{CH}_{3}$ ), 26.8 (C-12), 26.9 (C-15), 28.0 (C-23), 29.4 (C-20), 29.8 (C-16), 33.6 (glutaryl C-4'), 33.7 (glutaryl C-2'), 34.2 (C-7), 34.7 (C-22), 37.0 (C-10), 37.2 (C-13), 37.8 (C-4), 38.4 (C-1), 40.5 (28-Odimethylsuccinyl C-3"), 40.9 (C-8), 42.9 (C-14), 44.4 (28-O-dimethylsuccinyl C-2"), 44.5 (C-19), 46.4 (C-17), 48.2 (C-18), 50.0 (C-9), 55.3 (C-5), 63.1 (C-28), $76.9\left(\mathrm{CH}(\mathrm{Ph})_{2}\right), 81.0$ (C-3), 127.0 (benzhydryl C-2', 6'), 127.9 (benzyhydryl C-4'), 128.5 (benzhydryl C-3', 5'), 140.2 (benzhydryl C-1'), 171.5 (28-O-dimethylsuccinyl 1"-COO-), 171.9 (3-O-glutaryl 5'COO-), 172.6 (3-O-glutaryl 1'-COO-), 182.6 (28-O-dimethylsuccinyl COOH). HRESIMS (positive) $\mathrm{m} / z$ 875.5408 $[\mathrm{M}+\mathrm{Na}]^{+}$(calcd for $\mathrm{C}_{54} \mathrm{H}_{76} \mathrm{O}_{8} \mathrm{Na}, 875.5438$ ).

Compound 31: $[\alpha]_{\mathrm{D}}^{27}-7.4^{\circ}\left(c 1.26, \mathrm{CHCl}_{3}\right) .{ }^{1} \mathrm{H} \mathrm{NMR}\left(\mathrm{CDCl}_{3}, 400 \mathrm{MHz}\right) \delta 0.79,0.85$ (each $3 \mathrm{H}, \mathrm{d}, J=8.0 \mathrm{~Hz}, \mathrm{CH}_{3}-29,30$ ), 0.84 (each $\left.3 \mathrm{H}, \mathrm{s}, \mathrm{CH}_{3}-23,24\right), 0.87$ (3H, s, $\mathrm{CH}_{3}-25$ ), 0.96 $\left(3 \mathrm{H}, \mathrm{s}, \mathrm{CH}_{3}-27\right), 1.05\left(3 \mathrm{H}, \mathrm{s}, \mathrm{CH}_{3}-26\right), 1.31\left(6 \mathrm{H}, \mathrm{s}, 28-O\right.$-dimethylsuccinyl $\left.\mathrm{CH}_{3}\right), 2.00(2 \mathrm{H}$, quint, $J=7.2 \mathrm{~Hz}, 3-O$-glutaryl $\left.\mathrm{H}_{2}-3^{\prime}\right), 2.36\left(2 \mathrm{H}, \mathrm{t}, J=7.2 \mathrm{~Hz}, 3\right.$ - $O$-glutaryl $\left.\mathrm{H}_{2}-2^{\prime}\right), 2.50(2 \mathrm{H}$, $\mathrm{t}, J=7.2 \mathrm{~Hz}, 3$ - $O$-glutaryl $\left.\mathrm{H}_{2}-4^{\prime}\right), 2.65\left(2 \mathrm{H}, \mathrm{s}, 28\right.$ - $O$-dimethylsuccinyl $\left.\mathrm{H}_{2}-3 "\right), 3.81,4.32$ (each $\left.1 \mathrm{H}, \mathrm{d}, J=10.8 \mathrm{~Hz}, \mathrm{H}_{2}-28\right), 4.49(1 \mathrm{H}, \mathrm{dd}, J=6.0,10.0 \mathrm{~Hz}, \mathrm{H}-3), 6.90(1 \mathrm{H}, \mathrm{s}$, $\left.\mathrm{CH}(\mathrm{Ph})_{2}\right), 7.26-7.34\left(10 \mathrm{H}, \mathrm{m}\right.$, aromatic-H); ${ }^{13} \mathrm{C} \mathrm{NMR}\left(\mathrm{CDCl}_{3}, 100 \mathrm{MHz}\right) \delta 14.7(\mathrm{C}-27)$, 
14.9 (C-29), 16.1 (C-25, C-26), 16.6 (C-24), 18.2 (C-6), 20.4 (glutaryl C-3'), 20.8 (C-11), 21.6 (C-21), 22.9 (C-30), 23.7 (C-2), 25.4, 25.5 (28-O-dimethylsuccinyl $\mathrm{CH}_{3}$ ), $26.8(\mathrm{C}-12)$, 26.9 (C-15), 28.0 (C-23), 29.4 (C-20), 29.9 (C-16), 33.6 (glutaryl C-4'), 33.7 (glutaryl C-2'), 34.2 (C-7), 34.7 (C-22), 37.0 (C-10), 37.2 (C-13), 37.8 (C-4), 38.4 (C-1), 40.8 (28-Odimethylsuccinyl C-2"), 40.9 (C-8), 42.9 (C-14), 43.9 (28-O-dimethylsuccinyl C-3"), 44.6 (C-19), 46.7 (C-17), 48.2 (C-18), 50.0 (C-9), 55.4 (C-5), $63.4(\mathrm{C}-28), 77.0\left(\mathrm{CH}(\mathrm{Ph})_{2}\right), 81.0$ (C-3), 127.0 (benzhydryl C-2', 6'), 127.9 (benzyhydryl C-4'), 128.5 (benzhydryl C-3', 5'), 140.2 (benzhydryl C-1'), 171.9 (3-O-glutaryl 5'-COO-), 172.6 (3-O-glutaryl 1'-COO-), 175.8 (28-O-dimethylsuccinyl 1"-COOH), 177.1 (28- $O$-dimethylsuccinyl COO-).

HRESIMS (positive) $\mathrm{m} / z 875.5450[\mathrm{M}+\mathrm{Na}]^{+}$(calcd for $\mathrm{C}_{54} \mathrm{H}_{76} \mathrm{O}_{8} \mathrm{Na}, 875.5438$ ).

3-O-(5'-Benzhydryloxyglutaryl)-28- $\boldsymbol{O}$-glutaryldihydrobetulin (32): Yield 94.9\% (starting from $89 \mathrm{mg}$ of 20 and glutaric anhydride); colorless oil. $[\alpha]_{\mathrm{D}}^{27}-1.2^{\circ}\left(c 1.04, \mathrm{CHCl}_{3}\right) .{ }^{1} \mathrm{H}$ NMR $\left(\mathrm{CDCl}_{3}, 400 \mathrm{MHz}\right) \delta 0.78,0.84$ (each $\left.3 \mathrm{H}, \mathrm{d}, J=8.0 \mathrm{~Hz}, \mathrm{CH}_{3}-29,30\right), 0.83(3 \mathrm{H}, \mathrm{s}$, $\left.\mathrm{CH}_{3}-24\right), 0.84$ (3H, s, $\left.\mathrm{CH}_{3}-24\right), 0.87\left(3 \mathrm{H}, \mathrm{s}, \mathrm{CH}_{3}-25\right), 0.96\left(3 \mathrm{H}, \mathrm{s}, \mathrm{CH}_{3}-27\right), 1.06(3 \mathrm{H}, \mathrm{s}$, $\left.\mathrm{CH}_{3}-26\right), 1.98\left(2 \mathrm{H}\right.$, quint, $J=72 \mathrm{~Hz}, 28-O$-glutaryl $\left.\mathrm{H}_{2}-3 "\right), 2.00(2 \mathrm{H}$, quint, $J=7.2 \mathrm{~Hz}, 3-$ $O$-glutaryl $\left.\mathrm{H}_{2}-3^{\prime}\right), 2.36\left(2 \mathrm{H}, \mathrm{t}, J=7.2 \mathrm{~Hz}, 3-O\right.$-glutaryl $\left.\mathrm{H}_{2}-2^{\prime}\right), 2.43(2 \mathrm{H}, \mathrm{t}, J=7.2 \mathrm{~Hz}, 28-O$ glutaryl $\left.\mathrm{H}_{2}-2 "\right), 2.44\left(2 \mathrm{H}, \mathrm{t}, J=72 \mathrm{~Hz}, 28-O\right.$-glutaryl $\left.\mathrm{H}_{2}-4 "\right), 2.50(2 \mathrm{H}, \mathrm{t}, J=72 \mathrm{~Hz}, 3-O-$ glutaryl $\left.\mathrm{H}_{2}-4^{\prime}\right), 3.84,4.30$ (each $\left.1 \mathrm{H}, \mathrm{d}, J=10.8 \mathrm{~Hz}, \mathrm{H}_{2}-28\right), 4.49(1 \mathrm{H}, \mathrm{dd}, J=6.0,10.4 \mathrm{~Hz}$, $\mathrm{H}-3), 6.90\left(1 \mathrm{H}, \mathrm{s}, \mathrm{CH}(\mathrm{Ph})_{2}\right), 7.26-7.33(10 \mathrm{H}, \mathrm{m}$, aromatic-H $) ;{ }^{13} \mathrm{C} \mathrm{NMR}\left(\mathrm{CDCl}_{3}, 100 \mathrm{MHz}\right)$ $\delta 14.6$ (C-27), 14.9 (C-29), 16.1 (C-25, C-26), 16.6 (C-24), 18.2 (C-6), 20.0 (28-O-glutaryl C-3"), 20.4 (3-O-glutaryl C-3'), 20.8 (C-11), 21.6 (C-21), 22.9 (C-30), 23.7 (C-2), 26.8 (C-12), 27.0 (C-15), 28.0 (C-23), 29.4 (C-20), 29.9 (C-16), 32.8 (28-O-glutaryl C-4"), 33.3 (28-O-glutaryl C-2"), 33.6 (3-O-glutaryl C-4'), 33.7 (3-O-glutaryl C-2'), 34.2 (C-7), 34.7 (C-22), 37.1 (C-10), 37.2 (C-13), 37.8 (C-4), 38.4 (C-1), 41.0 (C-8), 42.9 (C-14), 44.6 (C-19), 46.6 (C-17), 48.2 (C-18), 50.0 (C-9), $55.4(\mathrm{C}-5), 62.9$ (C-28), $77.0\left(\mathrm{CH}(\mathrm{Ph})_{2}\right), 81.0$ (C-3), 127.1 (benzhydryl C-2', 6'), 127.9 (benzyhydryl C-4'), 128.5 (benzhydryl C-3', 5'), 140.2 (benzhydryl C-1'), 171.9 (3-O-glutaryl 5'-COO-), 172.6 (3-O-glutaryl 1'-COO-), 173.3 (28-O-glutaryl 1"-COO-), 177.3 (28-O-glutaryl COOH). HRESIMS (positive) $\mathrm{m} / z$ $861.5292[\mathrm{M}+\mathrm{Na}]^{+}$(calcd for $\mathrm{C}_{53} \mathrm{H}_{74} \mathrm{O}_{8} \mathrm{Na}, 861.5281$ ).

3-O-(5'-Benzhydryloxyglutaryl)-28-O-(3",3"-dimethylglutaryl)dihydrobetulin (33): Yield $91.6 \%$ (starting from $82 \mathrm{mg}$ of $\mathbf{2 0}$ and 3,3-dimethylglutaric anhydride); colorless oil. $[\alpha]_{\mathrm{D}}^{27}-5.4^{\circ}\left(c 1.43, \mathrm{CHCl}_{3}\right) .{ }^{1} \mathrm{H} \mathrm{NMR}\left(\mathrm{CDCl}_{3}, 400 \mathrm{MHz}\right) \delta 0.78,0.84($ each $3 \mathrm{H}, \mathrm{d}, J=8.0$ $\left.\mathrm{Hz}, \mathrm{CH}_{3}-29,30\right), 0.83\left(3 \mathrm{H}, \mathrm{s}, \mathrm{CH}_{3}-24\right), 0.84\left(3 \mathrm{H}, \mathrm{s}, \mathrm{CH}_{3}-24\right), 0.87$ (3H, s, $\left.\mathrm{CH}_{3}-25\right), 0.97$ $\left(3 \mathrm{H}, \mathrm{s}, \mathrm{CH}_{3}-27\right), 1.06\left(3 \mathrm{H}, \mathrm{s}, \mathrm{CH}_{3}-26\right), 1.16\left(6 \mathrm{H}, \mathrm{s}, 28-O\right.$-dimethylglutaryl $\left.\mathrm{CH}_{3}\right), 2.00(2 \mathrm{H}$, quint, $J=7.2 \mathrm{~Hz}, 3-O$-glutaryl $\left.\mathrm{H}_{2}-3^{\prime}\right), 2.36\left(2 \mathrm{H}, \mathrm{t}, J=7.2 \mathrm{~Hz}, 3-O\right.$-glutaryl $\left.\mathrm{H}_{2}-2^{\prime}\right), 2.46(2 \mathrm{H}$, s, 28-O-dimthylglutaryl $\left.\mathrm{H}_{2}-2 "\right), 2.49\left(2 \mathrm{H}, \mathrm{s}, 28-O\right.$-glutaryl $\left.\mathrm{H}_{2}-4 "\right), 2.50(2 \mathrm{H}, \mathrm{t}, J=7.2 \mathrm{~Hz}, 3$ $O$-glutaryl $\mathrm{H}_{2}-4$ '), 3.84, 4.30 (each $\left.1 \mathrm{H}, \mathrm{d}, J=10.8 \mathrm{~Hz}, \mathrm{H}_{2}-28\right), 4.49(1 \mathrm{H}, \mathrm{dd}, J=5.6,10.8$ $\mathrm{Hz}, \mathrm{H}-3), 6.90\left(1 \mathrm{H}, \mathrm{s}, \mathrm{CH}(\mathrm{Ph})_{2}\right), 7.26-7.33\left(10 \mathrm{H}, \mathrm{m}\right.$, aromatic-H); ${ }^{13} \mathrm{C} \mathrm{NMR}\left(\mathrm{CDCl}_{3}, 100\right.$ MHz) $\delta 14.7$ (C-27), 14.9 (C-29), 16.1 (C-25, C-26), 16.6 (C-24), 18.2 (C-6), 20.4 (3-Oglutaryl C-3'), 20.8 (C-11), 21.6 (C-21), 22.9 (C-30), 23.7 (C-2), 26.8 (C-12), 26.9 (C-15), 27.9 (28-O-dimethylglutaryl $\left.\mathrm{CH}_{3}\right), 28.0(\mathrm{C}-23), 29.4$ (C-20), 29.9 (C-16), 32.7 (28-Odimethylglutaryl C-3"), 33.6 (3-O-glutaryl C-4'), 33.7 (3-O-glutaryl C-2'), 34.2 (C-7), 34.7 (C-22), 37.1 (C-10), 37.2 (C-13), 37.8 (C-4), 38.4 (C-1), 41.0 (C-8), 42.9 (C-14), 44.6 (C-19), 45.1 (28-O-dimethylglutaryl C-4"), 45.4 (28-O-dimethylglutaryl C-2"), 46.4 (C-17), 48.2 (C-18), 50.0 (C-9), 55.4 (C-5), 63.1 (C-28), $77.0\left(\mathrm{CH}(\mathrm{Ph})_{2}\right), 81.0(\mathrm{C}-3), 127.1$ (benzhydryl C-2', 6'), 127.9 (benzyhydryl C-4'), 128.5 (benzhydryl C-3', 5'), 140.2 (benzhydryl C-1'), 171.9 (3-O-glutaryl 5'-COO-), 172.6 (3-O-glutaryl 1'-COO-), 173.0 (28$O$-dimethylglutaryl 1"-COO-), 175.6 (28- $O$-dimethylglutaryl $\mathrm{COOH}$ ). HRESIMS (positive) $\mathrm{m} / z 889.5605[\mathrm{M}+\mathrm{Na}]^{+}$(calcd for $\left.\mathrm{C}_{55} \mathrm{H}_{78} \mathrm{O}_{8} \mathrm{Na}, 889.5594\right)$. 
3-O-(5'-Benzhydryloxy-3',3'-dimethylglutaryl)-28-O-(3",,3"-

dimethylglutaryl)dihydrobetulin (34): Yield 97\% (starting from $137 \mathrm{mg}$ of $\mathbf{1 9}$ and 3,3-

dimethylglutaric anhydride); white solid. $[\alpha]_{\mathrm{D}}^{28}-9.3^{\circ}\left(c 2.94, \mathrm{CHCl}_{3}\right) .{ }^{1} \mathrm{H} \mathrm{NMR}\left(\mathrm{CDCl}_{3}, 400\right.$ $\mathrm{MHz}) \delta 0.78,0.83$ (each $\left.3 \mathrm{H}, \mathrm{d}, J=8.0 \mathrm{~Hz}, \mathrm{CH}_{3}-29,30\right), 0.81\left(3 \mathrm{H}, \mathrm{s}, \mathrm{CH}_{3}-24\right), 0.84(3 \mathrm{H}, \mathrm{s}$, $\left.\mathrm{CH}_{3}-24\right), 0.85\left(3 \mathrm{H}, \mathrm{s}, \mathrm{CH}_{3}-25\right), 0.96\left(3 \mathrm{H}, \mathrm{s}, \mathrm{CH}_{3}-27\right), 1.05\left(3 \mathrm{H}, \mathrm{s}, \mathrm{CH}_{3}-26\right), 1.09$ (6H, s, 3$O$-dimethylglutaryl $\left.\mathrm{CH}_{3}\right), 1.15\left(6 \mathrm{H}, \mathrm{s}, 28-O\right.$-dimethylglutaryl $\left.\mathrm{CH}_{3}\right), 2.36,2.43$ (each $1 \mathrm{H}, \mathrm{d}$, $J=14.4 \mathrm{~Hz}, 3$ - $O$-dimethylglutaryl $\mathrm{H}_{2}-2$ ) $, 2.46\left(2 \mathrm{H}, \mathrm{s}, 28\right.$ - $O$-dimthylglutaryl $\left.\mathrm{H}_{2}-2 "\right), 2.48$ $\left(2 \mathrm{H}, \mathrm{s}, 28-O\right.$-dimethylglutaryl $\mathrm{H}_{2}-4 "$ ), 2.55, 2.58 (each $1 \mathrm{H}, \mathrm{d}, J=14.4 \mathrm{~Hz}, 3-O$ dimethylglutaryl $\mathrm{H}_{2}-4^{\prime}$ ), 3.83, 4.29 (each $\left.1 \mathrm{H}, \mathrm{d}, J=11.2 \mathrm{~Hz}, \mathrm{H}_{2}-28\right), 4.47(1 \mathrm{H}, \mathrm{dd}, J=4.8$, $10.8 \mathrm{~Hz}, \mathrm{H}-3), 6.89\left(1 \mathrm{H}, \mathrm{s}, \mathrm{CH}(\mathrm{Ph})_{2}\right), 7.25-7.34\left(10 \mathrm{H}, \mathrm{m}\right.$, aromatic-H); ${ }^{13} \mathrm{C} \mathrm{NMR}\left(\mathrm{CDCl}_{3}\right.$, $100 \mathrm{MHz}) \boldsymbol{\delta} 14.6$ (C-27), 14.9 (C-29), 16.0 (C-25, C-26), 16.6 (C-24), 18.1 (C-6), 20.8 (C-11), 21.6 (C-21), 22.9 (C-30), 23.7 (C-2), 26.8 (C-12, C-15), 27.6, 27.7 (3-Odimethylglutaryl $\mathrm{CH}_{3}$ ), 27.9 (28-O-dimethylglutaryl $\mathrm{CH}_{3}$ ), $28.0(\mathrm{C}-23), 29.4(\mathrm{C}-20), 29.9$ (C-16), 32.6 (28-O-dimethylglutaryl C-3"), 32.8 (3-O-dimethylglutaryl C-3'), 34.2 (C-7), 34.7 (C-22), 37.0 (C-10), 37.2 (C-13), 37.6 (C-4), 38.4 (C-1), 41.0 (C-8), 42.8 (C-14), 44.5 (C-19), 45.1 (28-O-dimethylglutaryl C-4"), 45.3 (28- $O$-dimethylglutaryl C-2"), 45.4 (3-Odimethylglutaryl C-4'), 45.8 (3-O-dimethylglutaryl C-2'), 46.4 (C-17), 48.1 (C-18), 49.9 (C-9), 55.3 (C-5), $63.0(\mathrm{C}-28), 76.7\left(\mathrm{CH}(\mathrm{Ph})_{2}\right), 80.9$ (C-3), 127.1 (benzhydryl C-2', 6'), 127.8 (benzyhydryl C-4'), 128.4 (benzhydryl C-3', 5'), 140.2, 140.3 (benzhydryl C-1'), 170.8 (3-O-dimethylglutaryl 5'-COO-), 171.7 (3-O-dimethylglutaryl 1'-COO-), 172.9 (28-Odimethylglutaryl 1"-COO-), 176.4 (28-O-dimethylglutaryl $\mathrm{COOH})$. HRESIMS (positive) $m / z 917.5843[\mathrm{M}+\mathrm{Na}]^{+}$(calcd for $\mathrm{C}_{57} \mathrm{H}_{82} \mathrm{O}_{8} \mathrm{Na}, 917.5907$ ).

4.2.8 General procedure for coupling of 3,28-di- $O$-acylbetulin derivatives with AZT (35-48)-To a solution of 3,28-di- $O$-acylbetulin derivatives ( 1 equiv) in $\mathrm{CH}_{2} \mathrm{Cl}_{2}$ (4$10 \mathrm{~mL}$ ) was added DMAP ( 2 equiv), DCC (2 equiv) and 3'-azido-3'-dexoythymidine (AZT, 2 equiv). The reaction mixture was kept stirring at room temperature for overnight. After filtration, the filtrate was concentrated and the residue was chromatographed over silica gel column (hexane/EtOAc $=2: 1$ ) or purified by HPLC.

3'-Azido-3'-deoxythymidine 5'-yl 3-O-(4'-benzhydryloxy-3',3'-dimethylsuccinyl)lup-20(29)en-28-yl succinate (35): Yield 41.7\% (starting from $80 \mathrm{mg}$ of 21); white solid.

$[\alpha]_{\mathrm{D}}^{18}+6.2^{\circ}\left(c 0.53, \mathrm{CHCl}_{3}\right) .{ }^{1} \mathrm{H} \mathrm{NMR}\left(\mathrm{CDCl}_{3}, 500 \mathrm{MHz}\right) \delta 0.72\left(3 \mathrm{H}, \mathrm{s}, \mathrm{CH}_{3}-24\right), 0.76(3 \mathrm{H}$, $\left.\mathrm{s}, \mathrm{CH}_{3}-23\right), 0.77\left(3 \mathrm{H}, \mathrm{s}, \mathrm{CH}_{3}-25\right), 0.95\left(3 \mathrm{H}, \mathrm{s}, \mathrm{CH}_{3}-27\right), 1.01\left(3 \mathrm{H}, \mathrm{s}, \mathrm{CH}_{3}-26\right), 1.29,1.30$ (each $3 \mathrm{H}, \mathrm{s}, 3-\mathrm{O}$-dimethylsuccinyl $\left.\mathrm{CH}_{3}\right), 1.68\left(3 \mathrm{H}, \mathrm{s}, \mathrm{CH}_{3}-30\right), 1.95\left(3 \mathrm{H}, \mathrm{br} \mathrm{s}, \mathrm{AZT}-\mathrm{CH}_{3}\right)$, 2.33-2.50 (3H, m, AZT- $\mathrm{H}_{2}-2^{\prime}$ and $\left.\mathrm{H}-19\right), 2.62-2.79\left(4 \mathrm{H}, \mathrm{m}, 28-O\right.$-succinyl $\left.\mathrm{H}_{2}-2 ", 3 "\right), 2.64$, 2.68 (each $1 \mathrm{H}, \mathrm{d}, J=16.0 \mathrm{~Hz}, 3-O$-dimethylsuccinyl $\mathrm{H}_{2}-2^{\prime}$ ), 3.86, 4.30 (each $1 \mathrm{H}, \mathrm{d}, J=11.2$ $\left.\mathrm{Hz}, \mathrm{H}_{2}-28\right), 4.05$ (1H, q-like, AZT-H-4'), 4.21-4.24 (1H, m, AZT-H-3'), 4.30 (1H, dd, $J=$ $3.4,12.0 \mathrm{~Hz}$, AZT- $\left.\mathrm{H}_{2}-5 \mathrm{a}^{\prime}\right), 4.52\left(1 \mathrm{H}, \mathrm{dd}, J=4.0,12.0 \mathrm{~Hz}, \mathrm{AZT}-\mathrm{H}_{2}-5 \mathrm{~b}^{\prime}\right), 4.42(1 \mathrm{H}, \mathrm{dd}, J=$ $5.2,11.2 \mathrm{~Hz}, \mathrm{H}-3), 4.59,4.68$ (each $1 \mathrm{H}$, br s, $\left.\mathrm{H}_{2}-29\right), 6.15$ (1H, t-like, $J=6.3 \mathrm{~Hz}$, AZTH-1'), $6.84\left(1 \mathrm{H}, \mathrm{s}, \mathrm{CH}(\mathrm{Ph})_{2}\right), 7.24-7.32(11 \mathrm{H}, \mathrm{m}$, aromatic-H and AZT-H-6), $8.13(1 \mathrm{H}$, br s, AZT-NH); ${ }^{13} \mathrm{C}$ NMR $\left(\mathrm{CDCl}_{3}, 125 \mathrm{MHz}\right) \delta 12.6$ (AZT-CH3), 14.7 (C-27), 16.0 (C-26), 16.1 (C-25), 16.5 (C-24), 18.1 (C-6), 19.1 (C-30), 20.7 (C-11), 23.5 (C-2), 25.1 (C-12), 25.2, 25.4 (3-O-dimethylsuccinyl $\mathrm{CH}_{3}$ ), 27.0 (C-15), 27.9 (C-23), 29.0 (28-O-succinyl C-2", 3"), 29.5 (C-21), 29.7 (C-16), 34.1 (C-7), 34.5 (C-22), 37.0 (C-10), 37.6 (AZT-C-2'), 37.6 (C-13), 37.7 (C-4), 38.3 (C-1), 40.7 (dimethylsuccinyl C-3'), 40.8 (C-8), 42.7 (C-14), 44.5 (3-Odimethylsuccinyl C-2'), 46.4 (C-17), 47.7 (C-19), 48.7 (C-18), 50.2 (C-9), 55.3 (C-5), 60.1 (AZT-C-3'), 63.1 (AZT-C-5'), $63.3(\mathrm{C}-28), 77.0\left(\mathrm{CH}(\mathrm{Ph})_{2}\right), 81.3$ (C-3), 81.9 (AZT-C-4'), 85.1 (AZT-C-1'), 110.0 (C-29), 111.3 (AZT-C-5), 127.0, 127.1 (benzhydryl C-2', 6'), 127.7 (benzyhydryl C-4'), 128.4 (benzhydryl C-3', 5'), 135.3 (AZT-C-6), 140.3 (benzhydryl C-1'), 149.8 (AZT-C-2), 150.0 (C-20), 163.1 (AZT-C-4), 170.9 (3-O-dimethylsuccinyl 1'-COO-), 172.0 (28-O-succinyl 4"-COO-AZT), 172.6 (28-O-succinyl 1"-COO-), 175.4 (3-O- 
dimethylsuccinyl 4'-COO-). HRESIMS (positive) $\mathrm{m} / \mathrm{z} 1108.6014[\mathrm{M}+\mathrm{Na}]^{+}$(calcd for $^{-}$ $\left.\mathrm{C}_{6} 3 \mathrm{H}_{83} \mathrm{~N}_{5} \mathrm{O}_{11} \mathrm{Na}, 1108.5987\right)$.

1-(3'-Azido-3'-deoxythymidine-5'-yl)-4-[3-O-(4'-benzhydryloxy-3',3'-dimethylsuccinyl)lu p-20(29)en-28-yl] 2,2-dimethylsuccinate (36) and 4-(3'-Azido-3'-deoxythymidine-5'yl)-1-[3-O-(4'-benzhydryloxy-3',3'-dimethylsuccinyl)-lup-2 0(29)en-28-yl] 2,2-

dimethylsuccinate (37): Total yield 49.3\% (starting from $296 \mathrm{mg}$ of mixture of $\mathbf{2 2}$ and 23); used for next reaction without separation. A small amount of sample mixture was separated by HPLC (cholester, $\mathrm{CH}_{3} \mathrm{OH} / \mathrm{H}_{2} \mathrm{O}=97: 3$ ) for spectroscopic analyses.

Compound 36: $[\alpha]_{\mathrm{n}}^{27}+7.2^{\circ}\left(c 1.77 \mathrm{CHCl}_{3}\right) .{ }^{1} \mathrm{H} \mathrm{NMR}\left(\mathrm{CDCl}_{3}, 400 \mathrm{MHz}\right) \delta 0.74(3 \mathrm{H}, \mathrm{s}$, $\mathrm{CH}_{3}$-24), 0.78 (each $\left.3 \mathrm{H}, \mathrm{s}, \mathrm{CH}_{3}-23,25\right), 0.96$ (3H, s, $\left.\mathrm{CH}_{3}-27\right), 1.02$ (3H, s, $\left.\mathrm{CH}_{3}-26\right), 1.30$, 1.31 (each $3 \mathrm{H}, \mathrm{s}, 28-O$-dimethylsuccinyl $\mathrm{CH}_{3}$ ), 1.31, 1.32 (each $3 \mathrm{H}, \mathrm{s}, 3-O-$

dimethylsuccinyl $\mathrm{CH}_{3}$ ), 1.68 (3H, s, CH3-30), 1.94 (3H, br s, AZT-CH3), 2.26-2.33, 2.442.50 (each 1H, m, AZT- $H_{2}-2^{\prime}$ ), 2.37-2.44 (1H, m, H-19), 2.65, 2.69 (each 1H, d, $J=16.0$ $\mathrm{Hz}, 3-O$-dimethylsuccinyl $\mathrm{H}_{2}-2^{\prime}$ ), 2.61, 2.75 (each $1 \mathrm{H}, \mathrm{d}, J=16.4 \mathrm{~Hz}, 28-O$ dimethylsuccinyl $\mathrm{H}_{2}-2$ "), 3.84, 4.29 (each $1 \mathrm{H}, \mathrm{d}, J=10.8 \mathrm{~Hz}, \mathrm{H}_{2}-28$ ), 4.09 (1H, q-like, AZT-H-4'), 4.26-4.30 (1H, m, AZT-H-3'), 4.30, 4.52 (each 1H, dd, $J=4.0,12.0 \mathrm{~Hz}$, AZT$\mathrm{H}_{2}-5$ '), $4.43(1 \mathrm{H}, \mathrm{dd}, J=5.2,10.8 \mathrm{~Hz}, \mathrm{H}-3), 4.60,4.69$ (each $1 \mathrm{H}$, br s, $\left.\mathrm{H}_{2}-29\right), 6.18(1 \mathrm{H}, \mathrm{t}-$ like, $J=6.4 \mathrm{~Hz}$, AZT-H-1'), $6.86\left(1 \mathrm{H}, \mathrm{s}, \mathrm{CH}(\mathrm{Ph})_{2}\right), 7.24-7.33(11 \mathrm{H}, \mathrm{m}$, aromatic-H and AZT-H-6), $8.56\left(1 \mathrm{H}\right.$, br s, AZT-NH); ${ }^{13} \mathrm{C}$ NMR $\left(\mathrm{CDCl}_{3}, 100 \mathrm{MHz}\right) \delta 12.5\left(\mathrm{AZT}-\mathrm{CH}_{3}\right), 14.7$ (C-27), 15.9 (C-26), 16.1 (C-25), 16.5 (C-24), 18.1 (C-6), 19.1 (C-30), 20.7 (C-11), 23.5 (C-2), 25.2 (C-12), 25.2, 25.4 (3-O-dimethylsuccinyl $\left.\mathrm{CH}_{3}\right), 25.1,25.9$ (28-Odimethylsuccinyl $\mathrm{CH}_{3}$ ), 27.0 (C-15), 27.9 (C-23), 29.5 (C-21), 29.7 (C-16), 34.1 (C-7), 34.5 (C-22), 37.0 (C-10), 37.4 (AZT-C-2'), 37.6 (C-4, 13), 38.3 (C-1), 40.7 (dimethylsuccinyl C-3', C-3"), 40.8 (C-8), 42.7 (C-14), 44.5 (3-O-dimethylsuccinyl C-2'), 44.6 (28- $O$ dimethylsuccinyl C-2"), 46.3 (C-17), 47.7 (C-19), 48.7 (C-18), 50.2 (C-9), 55.3 (C-5), 60.4 (AZT-C-3'), 63.1 (C-28), 63.0 (AZT-C-5'), $77.0\left(\mathrm{CH}(\mathrm{Ph})_{2}\right), 81.2$ (C-3), 81.9 (AZT-C-4'), 84.8 (AZT-C-1'), 110.0 (C-29), 111.4 (AZT-C-5), 127.0, 127.1 (benzhydryl C-2', 6'), 127.7 (benzyhydryl C-4'), 128.4 (benzhydryl C-3', 5'), 135.0 (AZT-C-6), 140.4 (benzhydryl C-1'), 149.9 (C-20 and AZT-C-2), 163.3 (AZT-C-4), 170.8 (3-O-dimethylsuccinyl 1'-COO-), 171.7 (28-O-dimethylsuccinyl 1"-COO-), 175.4 (3-O-dimethylsuccinyl 4'-COO-), 176.4 (28-O-dimethylsuccinyl 4"-COO-AZT). HRESIMS (positive) $\mathrm{m} / 2.1136 .6310[\mathrm{M}+\mathrm{Na}]^{+}$ (calcd for $\mathrm{C}_{65} \mathrm{H}_{87} \mathrm{~N}_{5} \mathrm{O}_{11} \mathrm{Na}, 1136.6300$ ).

Compound 37: $[\alpha]_{\mathrm{D}}^{27}+11.6^{\circ}\left(c 3.64 \mathrm{CHCl}_{3}\right) .{ }^{1} \mathrm{H} \mathrm{NMR}\left(\mathrm{CDCl}_{3}, 400 \mathrm{MHz}\right) \delta 0.73(3 \mathrm{H}, \mathrm{s}$, CH3-24), 0.77 (3H, s, $\left.\mathrm{CH}_{3}-23\right), 0.78(3 \mathrm{H}, \mathrm{s}, \mathrm{C}-25), 0.96\left(3 \mathrm{H}, \mathrm{s}, \mathrm{CH}_{3}-27\right), 1.02(3 \mathrm{H}, \mathrm{s}$, $\mathrm{CH}_{3}-26$ ), 1.30, 1.32 (each $3 \mathrm{H}, \mathrm{s}, 3$ - $O$-dimethylsuccinyl $\mathrm{CH}_{3}$ ), 1.31, 1.34 (each $3 \mathrm{H}, \mathrm{s}, 28-O$ dimethylsuccinyl $\left.\mathrm{CH}_{3}\right), 1.69\left(3 \mathrm{H}, \mathrm{s}, \mathrm{CH}_{3}-30\right), 1.95(3 \mathrm{H}, \mathrm{br}$ s, AZT-CH 3$), 2.33-2.50(3 \mathrm{H}, \mathrm{m}$, AZT- $\mathrm{H}_{2}-2^{\prime}$ and $\mathrm{H}-19$ ), 2.62, 2.66 (each $1 \mathrm{H}, \mathrm{d}, J=16.4 \mathrm{~Hz}, 28$ - $O$-dimethylsuccinyl $\mathrm{H}_{2}-3$ "), 2.64, 2.69 (each $1 \mathrm{H}, \mathrm{d}, J=16.0 \mathrm{~Hz}, 3-O$-dimethylsuccinyl $\mathrm{H}_{2}-2^{\prime}$ ), 3.85, 4.29 (each $1 \mathrm{H}, \mathrm{d}, J=$ $\left.10.8 \mathrm{~Hz}, \mathrm{H}_{2}-28\right), 4.04$ (1H, q-like, AZT-H-4'), 4.18-4.22(1H, m, AZT-H-3'), 4.26 (1H, dd, $J$ $=4.0,12.0 \mathrm{~Hz}$, AZT-H-5a'), $4.42(1 \mathrm{H}, \mathrm{dd}, J=4.4,12.0 \mathrm{~Hz}$, AZT-H-5b'), $4.43(1 \mathrm{H}, \mathrm{dd}, J=$ 4.8, $10.4 \mathrm{~Hz}, \mathrm{H}-3$ ), 4.60, 4.69 (each $1 \mathrm{H}$, br s, $\left.\mathrm{H}_{2}-29\right), 6.15$ (1H, t-like, $J=6.4 \mathrm{~Hz}$, AZT-H-1'), $6.85\left(1 \mathrm{H}, \mathrm{s}, \mathrm{CH}(\mathrm{Ph})_{2}\right), 7.24-7.32(11 \mathrm{H}, \mathrm{m}$, aromatic-H and AZT-H-6), $8.94(1 \mathrm{H}, \mathrm{br} \mathrm{s}$, AZT$\mathrm{NH}) ;{ }^{13} \mathrm{C} \mathrm{NMR}\left(\mathrm{CDCl}_{3}, 100 \mathrm{MHz}\right) \delta 12.6\left(\mathrm{AZT}-\mathrm{CH}_{3}\right), 14.7(\mathrm{C}-27), 15.9(\mathrm{C}-26), 16.0$ (C-25), 16.5 (C-24), 18.1 (C-6), 19.1 (C-30), 20.7 (C-11), 23.5 (C-2), 25.2 (C-12), 25.2, 25.5 (3-O-dimethylsuccinyl $\left.\mathrm{CH}_{3}\right), 25.4,25.8$ (28-O-dimethylsuccinyl $\left.\mathrm{CH}_{3}\right), 27.0(\mathrm{C}-15), 27.9$ (C-23), 29.5 (C-21), 29.8 (C-16), 34.1 (C-7), 34.5 (C-22), 37.0 (C-10), 37.5 (AZT-C-2'), $37.6(\mathrm{C}-4,13), 38.3$ (C-1), 40.7 (3-O-dimethylsuccinyl C-3'), 40.8 (C-8), 41.0 (28-Odimethylsuccinyl C-2"), 42.7 (C-14), 43.8 (28-O-dimethylsuccinyl C-3"), 44.5 (3-Odimethylsuccinyl C-2'), 46.5 (C-17), 47.7 (C-19), 48.8 (C-18), 50.2 (C-9), 55.3 (C-5), 60.3 
(AZT-C-3'), 62.9 (AZT-C-5'), 63.2 (C-28), $77.0\left(\mathrm{CH}(\mathrm{Ph})_{2}\right), 81.2$ (C-3), 81.7 (AZT-C-4'), 85.2 (AZT-C-1'), 109.9 (C-29), 111.3 (AZT-C-5), 127.0, 127.1 (benzhydryl C-2', 6'), 127.7 (benzyhydryl C-4'), 128.4 (benzhydryl C-3', 5'), 135.3 (AZT-C-6), 140.3 (benzhydryl C-1'), 150.0 (C-20 and AZT-C-2), 163.5 (AZT-C-4), 170.7 (28-O-dimethylsuccinyl 4"-COOAZT), 170.8 (3-O-dimethylsuccinyl 1'-COO-), 175.4 (3-O-dimethylsuccinyl 4'-COO-), 176.9 (28-O-dimethylsuccinyl 1"-COO-). HRESIMS (positive) $\mathrm{m} / z .1136 .6299[\mathrm{M}+\mathrm{Na}]^{+}$ (calcd for $\mathrm{C}_{65} \mathrm{H}_{87} \mathrm{~N}_{5} \mathrm{O}_{11} \mathrm{Na}, 1136.6300$ ).

3'-Azido-3'-deoxythymidine-5'-yl 3-O-(4'-benzhydryloxy-3',3'-dimethylsuccinyl)lup-20(29)en-28-yl glutarate (38): Yield 47.4\% (starting from $180 \mathrm{mg}$ of 24); white solid.

$[\alpha]_{\mathrm{D}}^{15}+24.2^{\circ}\left(c 0.5 \mathrm{CHCl}_{3}\right) .{ }^{1} \mathrm{H} \mathrm{NMR}\left(\mathrm{CDCl}_{3}, 500 \mathrm{MHz}\right) \delta 0.73\left(3 \mathrm{H}, \mathrm{s}, \mathrm{CH}_{3}-24\right), 0.77(3 \mathrm{H}$, $\left.\mathrm{s}, \mathrm{CH}_{3}-23\right), 0.78\left(3 \mathrm{H}, \mathrm{s}, \mathrm{CH}_{3}-25\right), 0.96\left(3 \mathrm{H}, \mathrm{s}, \mathrm{CH}_{3}-27\right), 1.01\left(3 \mathrm{H}, \mathrm{s}, \mathrm{CH}_{3}-26\right), 1.30,1.31$ (each 3H, s, 3-O-dimethylsuccinyl $\mathrm{CH}_{3}$ ), 1.68 (3H, s, CH3-30), 1.94 (3H, s, AZT-CH3), 1.99 (2H, quint, $J=7.0 \mathrm{~Hz}, 28$ - $O$-glutaryl $\left.\mathrm{H}_{2}-3 "\right), 2.42(2 \mathrm{H}, \mathrm{t}, J=7.0 \mathrm{~Hz}, 28$ - $O$-glutaryl $\left.\mathrm{H}_{2}-2^{\prime \prime}\right), 2.47$ (2H, t, $J=7.2 \mathrm{~Hz}, 28-O$-glutaryl $\left.\mathrm{H}_{2}-4 "\right), 2.33-2.50\left(3 \mathrm{H}, \mathrm{m}, \mathrm{AZT}-\mathrm{H}_{2}-2^{\prime}\right.$ and H-19), 2.64, 2.69 (each 1H, d, $J=16.0 \mathrm{~Hz}, 3$ - $O$-dimethylsuccinyl $\mathrm{H}_{2}-2$ '), 3.85, 4.28 (1H, d, $J$ $\left.=11.2 \mathrm{~Hz}, \mathrm{H}_{2}-28 \mathrm{a}\right), 4.06$ (1H, m, AZT-H-4'), 4.23 (1H, m, AZT-H-3'), 4.32 (1H, dd, $J=3.5$, $12.2 \mathrm{~Hz}$, AZT-H-5a'), 4.39 (1H, dd, $J=4.8,12.2 \mathrm{~Hz}$, AZT-H-5b'), 4.43 (1H, dd, $J=4.8$, $11.2 \mathrm{~Hz}, \mathrm{H}-3), 4.59,4.69$ (each $1 \mathrm{H}$, br s, $\left.\mathrm{H}_{2}-29\right)$, 6.10 (1H, t-like, $J=5.5 \mathrm{~Hz}$, AZT-H-1'), $6.85\left(1 \mathrm{H}, \mathrm{s}, \mathrm{CH}(\mathrm{Ph})_{2}\right), 7.21-7.32(11 \mathrm{H}, \mathrm{m}$, aromatic-H and AZT-H-6), $9.37(1 \mathrm{H}$, br s, AZT$\mathrm{NH}) ;{ }^{13} \mathrm{C} \mathrm{NMR}\left(\mathrm{CDCl}_{3}, 125 \mathrm{MHz}\right){ }^{13} \mathrm{C} \mathrm{NMR}\left(\mathrm{CDCl}_{3}, 125 \mathrm{MHz}\right) \delta 12.6\left(\mathrm{AZT}_{-} \mathrm{CH}_{3}\right), 14.7$ (C-27), 15.9 (C-26), 16.0 (C-25), 16.4 (C-24), 18.0 (C-6), 19.0 (C-30), 19.9 (28-O-glutaryl C-3"), 20.7 (C-11), 23.5 (C-2), 25.0 (C-12), 25.1, 25.3 (3-O-dimethylsuccinyl $\mathrm{CH}_{3}$ ), 26.9 (C-15), 27.8 (C-23), 29.4 (C-21), 29.7 (C-16), 33.0 (28-O-glutaryl C-4"), 33.1 (28-Oglutaryl C-2"), 34.0 (C-7), 34.4 (C-22), 36.9 (C-10), 37.4 (AZT-C-2'), 37.5 (C-13), 37.6 (C-4), 38.3 (C-1), 40.6 (dimethylsuccinyl C-3'), 40.8 (C-8), 42.6 (C-14), 44.4 (3-Odimethylsuccinyl C-2'), 46.3 (C-17), 47.6 (C-19), 48.7 (C-18), 50.1 (C-9), 55.2 (C-5), 60.5 (AZT-C-3'), 62.8 (C-28), 63.3 (AZT-C-5'), $76.9\left(\mathrm{CH}(\mathrm{Ph})_{2}\right), 81.2$ (C-3), 81.6 (AZT-C-4'), 85.6 (AZT-C-1'), 109.9 (C-29), 111.2 (AZT-C-5), 126.9, 127.0 (benzhydryl C-2', 6'), 127.7 (benzyhydryl C-4'), 128.3 (benzhydryl C-3', 5'), 135.4 (AZT-C-6), 140.2, 140.3 (benzhydryl C-1'), 149.9 (C-20), 150.0 (AZT-C-2), 163.4 (AZT-C-4), 170.8 (3-O-dimethylsuccinyl 1'COO-), 172.3 (28-O-glutaryl 5"-COO-AZT), 173.1 (28-O-glutaryl 1"-COO-), 175.3 (3-Odimethylsuccinyl 4'-COO-). HRESIMS (positive) $\mathrm{m} / \mathrm{z} 1122.6141[\mathrm{M}+\mathrm{Na}]^{+}$(calcd for $\left.\mathrm{C}_{64} \mathrm{H}_{85} \mathrm{~N}_{5} \mathrm{O}_{11} \mathrm{Na}, 1122.6143\right)$.

3'-Azido-3'-deoxythymidine-5'-yl 3-O-(4'-benzhydryloxy-3',3'-dimethylsuccinyl)lup-20(29)en-28-yl 3,3-dimethylglutarate (39): Yield 74.2\% (starting from $120 \mathrm{mg}$ of 25); white solid. $[\alpha]_{\mathrm{D}}^{26}+14.1^{\circ}\left(c 2.55 \mathrm{CHCl}_{3}\right) .{ }^{1} \mathrm{H} \mathrm{NMR}\left(\mathrm{CDCl}_{3}, 400 \mathrm{MHz}\right) \delta 0.74(3 \mathrm{H}, \mathrm{s}$, $\left.\mathrm{CH}_{3}-24\right), 0.78$ (each $\left.3 \mathrm{H}, \mathrm{s}, \mathrm{CH}_{3}-23,25\right), 0.96\left(3 \mathrm{H}, \mathrm{s}, \mathrm{CH}_{3}-27\right), 1.01$ (3H, s, $\left.\mathrm{CH}_{3}-26\right), 1.13$, 1.16 (each $3 \mathrm{H}, \mathrm{s}, 28$ - $O$-dimethylglutaryl $\mathrm{CH}_{3}$ ), 1.30, 1.31 (each $3 \mathrm{H}, \mathrm{s}, 3$ - $O$-dimethylsuccinyl $\left.\mathrm{CH}_{3}\right), 1.69$ (3H, s, $\left.\mathrm{CH}_{3}-30\right), 1.95$ (3H, br s, AZT-CH $), 2.30-2.35,2.40-2.51$ (2H, m, AZT$\mathrm{H}_{2}-2^{\prime}$ ), 2.35-2.42 (1H, m, H-19), 2.43, 2.50 (each $1 \mathrm{H}, \mathrm{d}, J=16.0 \mathrm{~Hz}, 28$ - $O$-dimethylglutaryl $\mathrm{H}_{2}-2^{\prime \prime}$ ), 2.49, 2.56 (each $1 \mathrm{H}, \mathrm{d}, J=16.0 \mathrm{~Hz}, 28$-O-dimethylglutaryl $\mathrm{H}_{2}-4$ "), 2.65, 2.69 (each $1 \mathrm{H}, \mathrm{d}, J=16.0 \mathrm{~Hz}, 3-O$-dimethylsuccinyl $\mathrm{H}_{2}-2^{\prime}$ ), 3.82, 4.26 (each $1 \mathrm{H}, \mathrm{d}, J=10.8 \mathrm{~Hz}$, $\mathrm{H}_{2}$-28), 4.07 (1H, q-like, AZT-H-4'), 4.20-4.25 (1H, m, AZT-H-3'), 4.27 (1H, dd, J = 3.6, $12.0 \mathrm{~Hz}$, AZT-H-5a'), 4.44 (1H, dd, $J=4.4,12.0 \mathrm{~Hz}$, AZT-H-5b'), 4.44 (1H, dd, $J=4.4$, $12.0 \mathrm{~Hz}, \mathrm{H}-3), 4.60,4.69$ (each $1 \mathrm{H}$, br s, $\left.\mathrm{H}_{2}-29\right)$, 6.15 (1H, t-like, $J=6.4 \mathrm{~Hz}$, AZT-H-1'), $6.86\left(1 \mathrm{H}, \mathrm{s}, \mathrm{CH}(\mathrm{Ph})_{2}\right), 7.24-7.33(11 \mathrm{H}, \mathrm{m}$, aromatic-H and AZT-H-6), $8.78(1 \mathrm{H}$, br s, AZT$\mathrm{NH}) ;{ }^{13} \mathrm{C}$ NMR $\left(\mathrm{CDCl}_{3}, 100 \mathrm{MHz}\right) \delta 12.6\left(\mathrm{AZT}-\mathrm{CH}_{3}\right), 14.7(\mathrm{C}-27), 16.0(\mathrm{C}-26), 16.1$ (C-25), 16.5 (C-24), 18.1 (C-6), 19.1 (C-30), 20.8 (C-11), 23.5 (C-2), 25.2 (C-12), 25.2, 25.4 (3-O-dimethylsuccinyl $\left.\mathrm{CH}_{3}\right), 27.0(\mathrm{C}-15), 27.9$ (28-O-dimethylglutaryl $\left.\mathrm{CH}_{3}\right), 28.0(\mathrm{C}-23)$, 29.5 (C-21), 29.8 (C-16), 32.6 (28-O-dimethylglutaryl C-3"), 34.1 (C-7), 34.6 (C-22), 37.0 
(C-10), 37.5 (AZT-C-2'), 37.6 (C-4, 13), 38.3 (C-1), 40.7 (3-O-dimethylsuccinyl C-3'), 40.8 (C-8), 42.6 (C-14), 44.5 (3-O-dimethylsuccinyl C-2', 28-O-dimethylglutaryl C-4"), 45.0 (28$O$-dimethylglutaryl C-2"), 46.2 (C-17), 47.7 (C-19), 48.7 (C-18), 50.2 (C-9), 55.3 (C-5), 60.4 (AZT-C-3'), 62.6 (C-28), 62.7 (AZT-C-5'), $77.0\left(\mathrm{CH}(\mathrm{Ph})_{2}\right), 81.2$ (C-3), 81.8 (AZTC-4'), 85.2 (AZT-C-1'), 109.9 (C-29), 111.3 (AZT-C-5), 127.0, 127.1 (benzhydryl C-2', 6'), 127.7 (benzyhydryl C-4'), 128.4 (benzhydryl C-3', 5'), 135.2 (AZT-C-6), 140.3 (benzhydryl C-1'), 150.0 (C-20 and AZT-C-2), 163.4 (AZT-C-4), 170.8 (3-O-dimethylsuccinyl 1'-COO -), 171.1 (28-O-dimethylglutaryl 5"-COO-AZT), 172.3 (28-O-dimethylglutaryl 1"-COO-), 175.4 (3-O-dimethylsuccinyl 4'-COO-). HRESIMS (positive) $\mathrm{m} / z 1150.6467[\mathrm{M}+\mathrm{Na}]^{+}$ (calcd for $\mathrm{C}_{66} \mathrm{H}_{89} \mathrm{~N}_{5} \mathrm{O}_{11} \mathrm{Na}, 1150.6456$ ).

3'-Azido-3'-deoxythymidine-5'-yl 3-O-(4'-benzhydryloxy-3',3'-dimethylglutaryl)lup-20(29)en-28-yl 3,3-dimethylglutarate (40): Yield 58.8\% (starting from $143 \mathrm{mg}$ of 26); white solid. $[\alpha]_{\mathrm{D}}^{26}+13.5^{\circ}\left(c 4.13 \mathrm{CHCl}_{3}\right) .{ }^{1} \mathrm{H} \mathrm{NMR}\left(\mathrm{CDCl}_{3}, 400 \mathrm{MHz}\right) \delta 0.80(3 \mathrm{H}, \mathrm{s}$, $\left.\mathrm{CH}_{3}-24\right), 0.82\left(6 \mathrm{H}, \mathrm{s}, \mathrm{CH}_{3}-23,25\right), 0.97$ (3H, s, $\left.\mathrm{CH}_{3}-27\right), 1.02\left(3 \mathrm{H}, \mathrm{s}, \mathrm{CH}_{3}-26\right), 1.08$ (6H, s, 3-O-dimethylglutaryl $\mathrm{CH}_{3}$ ), $1.12,1.15$ (each $3 \mathrm{H}, \mathrm{s}, 28$ - $O$-dimethylglutaryl $\left.\mathrm{CH}_{3}\right), 1.68(3 \mathrm{H}$, s, $\left.\mathrm{CH}_{3}-30\right), 1.95$ (3H, br s, AZT-CH3), 2.32-2.50 (3H, m, AZT-H ${ }_{2}-2$ and H-19), 2.34, 2.41 (each $1 \mathrm{H}, \mathrm{d}, J=14.4 \mathrm{~Hz}, 3-O$-dimethylglutaryl $\mathrm{H}_{2}-2$ ) , 2.44, 2.50 (each $1 \mathrm{H}, \mathrm{d}, J=14.4 \mathrm{~Hz}$, 28-O-dimethylglutaryl $\mathrm{H}_{2}-2 "$ ), 2.49, 2.56 (each $1 \mathrm{H}, \mathrm{d}, J=14.4 \mathrm{~Hz}, 28$ - $O$-dimethylglutaryl $\mathrm{H}_{2}-3$ "), 2.54, 2.57 (each $1 \mathrm{H}, \mathrm{d}, J=14.4 \mathrm{~Hz}, 3$-O-dimethylglutaryl $\mathrm{H}_{2}-4^{\prime}$ ), 3.82, 4.26 (each $\left.1 \mathrm{H}, \mathrm{d}, J=11.2 \mathrm{~Hz}, \mathrm{H}_{2}-28\right), 4.06$ (1H, q-like, AZT-H-4'), 4.21-4.28 (1H, m, AZT-H-3'), 4.26 $(1 \mathrm{H}, \mathrm{dd}, J=3.6,12.0 \mathrm{~Hz}$, AZT-H-5a'), $4.43(1 \mathrm{H}, \mathrm{dd}, J=4.4,12.0 \mathrm{~Hz}$, AZT-H-5b'), 4.46 $(1 \mathrm{H}, \mathrm{dd}, J=4.8,11.2 \mathrm{~Hz}, \mathrm{H}-3), 4.59,4.69$ (each $1 \mathrm{H}$, br s, $\left.\mathrm{H}_{2}-29\right), 6.15$ (1H, t-like, $J=6.4$ $\left.\mathrm{Hz}, \mathrm{AZT}-\mathrm{H}-1^{\prime}\right), 6.88\left(1 \mathrm{H}, \mathrm{s}, \mathrm{CH}(\mathrm{Ph})_{2}\right), 7.24-7.33(11 \mathrm{H}, \mathrm{m}$, aromatic-H and AZT-H-6), 9.07 $\left(1 \mathrm{H}\right.$, br s, AZT-NH); ${ }^{13} \mathrm{C}$ NMR $\left(\mathrm{CDCl}_{3}, 100 \mathrm{MHz}\right) \delta 12.5$ (AZT-CH3), 14.7 (C-27), 15.9 (C-26), 16.1 (C-25), 16.6 (C-24), 18.1 (C-6), 19.1 (C-30), 20.7 (C-11), 23.7 (C-2), (C-12), 26.9 (C-15), 27.6, (3-O-dimethylglutaryl $\mathrm{CH}_{3}$ ), 27.8, 27.9 (28-O-dimethylglutaryl $\mathrm{CH}_{3}$ ), 27.9 (C-23), 29.5 (C-21), 29.8 (C-16), 32.5 (28-O-dimethylglutaryl C-3"), 32.8 (3-Odimethylglutaryl C-3'), 34.1 (C-7), 34.6 (C-22), 37.0 (C-10), 37.5 (AZT-C-2', C-13), 37.6 (C-4), 38.3 (C-1), 40.8 (C-8), 42.6 (C-14), 44.5 (28-O-dimethylglutaryl C-4"), 45.0 (28-Odimethylsuccinyl C-2"), 45.4 (3- $O$-dimethylglutaryl C-4'), 45.7 (3- $O$-dimethylglurayl C-2'), (C-17), 47.6 (C-19), 48.7 (C-18), 50.2 (C-9), 55.3 (C-5), 60.4 (AZT-C-3'), 62.6 (C-28), 62.7 (AZT-C-5'), $76.6\left(\mathrm{CH}(\mathrm{Ph})_{2}\right), 80.8$ (C-3), 81.7 (AZT-C-4'), 85.2 (AZT-C-1'), 109.9 (C-29), 111.3 (AZT-C-5), 127.1 (benzhydryl C-2', 6'), 127.7 (benzyhydryl C-4'), 128.4 (benzhydryl C-3', 5'), 135.2 (AZT-C-6), 140.2 (benzhydryl C-1'), 149.9 (C-20), 150.0 (AZT-C-2), 163.5 (AZT-C-4), 170.8 (3-O-dimethylglutaryl 5'-COO-), 171.1 (28-O-dimethylglutaryl 5"-COOAZT), 171.6 (3-O-dimethylglutaryl 1'-COO-), 172.2 (28-O-dimethylglutaryl 1"-COO-). HRESIMS (positive) $\mathrm{m} / 2$ 1164.6608 $[\mathrm{M}+\mathrm{Na}]^{+}$(calcd for $\mathrm{C}_{67} \mathrm{H}_{91} \mathrm{~N}_{5} \mathrm{O}_{11} \mathrm{Na}, 1164.6613$ ).

1-(3'-Azido-3'-deoxythymidine-5'-yl)-4-[3-O-(4'-benzhydryl-3',3'-dimethylsuccinyl)lup-2 8-yl] 2,2-dimethylsuccinate (41) and 4-(3'-Azido-3'-deoxythymidine-5'-yl)-1-[3-O(4'-benzhydryl-3',3'-dimethylsuccinyl)-lup-28-y 1] 2,2-dimethylsuccinate (42): Total yield $36.0 \%$ (starting from $285 \mathrm{mg}$ of mixture of $\mathbf{2 7}$ and $\mathbf{2 8}$ ); used for next reaction without purification. For spectroscopic analysis, a small amount of sample was further purified by preparative HPLC (cholester, $\mathrm{CH}_{3} \mathrm{OH} / \mathrm{H}_{2} \mathrm{O}=97: 3$ ); white solid.

Compound 41: $[\alpha]_{\mathrm{D}}^{30}-6.7^{\circ}\left(c 1.69 \mathrm{CHCl}_{3}\right) .{ }^{1} \mathrm{H} \mathrm{NMR}\left(\mathrm{CDCl}_{3}, 400 \mathrm{MHz}\right) \delta 0.77,0.84$ (each $3 \mathrm{H}, \mathrm{d}, J=8.0 \mathrm{~Hz}, \mathrm{CH} 3-29,30), 0.74\left(3 \mathrm{H}, \mathrm{s}, \mathrm{CH}_{3}-24\right), 0.78\left(3 \mathrm{H}, \mathrm{s}, \mathrm{CH}_{3}-23\right), 0.79$ (3H, s, $\left.\mathrm{CH}_{3}-25\right), 0.94\left(3 \mathrm{H}, \mathrm{s}, \mathrm{CH}_{3}-27\right), 1.01\left(3 \mathrm{H}, \mathrm{s}, \mathrm{CH}_{3}-26\right), 1.31(12 \mathrm{H}, \mathrm{s}, 3-O, 28-O-$ dimethylsuccinyl $\mathrm{CH}_{3}$ ), 1.94 (3H, br s, AZT-CH3), 2.25-2.32, 2.44-2.50 (each 1H, m, AZT- $\mathrm{H}_{2}-2^{\prime}$ ), 2.65, 2.70 (each $1 \mathrm{H}, \mathrm{d}, J=16.0 \mathrm{~Hz}, 3$ - $O$-dimethylsuccinyl $\left.\mathrm{H}_{2}-2^{\prime}\right), 2.59,2.75$ (each $1 \mathrm{H}, \mathrm{d}, J=16.4 \mathrm{~Hz}, 28$ - $O$-dimethylsuccinyl $\mathrm{H}_{2}$-2"), 3.81, 4.28 (each $1 \mathrm{H}, \mathrm{d}, J=11.2$ 
Hz, H2-28), 4.09 (1H, q-like, AZT-H-4'), 4.25-4.29 (1H, m, AZT-H-3'), 4.29 (1H, dd, $J=$ $4.4,12.0 \mathrm{~Hz}$, AZT-H-5a'), $4.52(1 \mathrm{H}, \mathrm{dd}, J=4.0,12.0 \mathrm{~Hz}$, AZT-H-5b'), $4.44(1 \mathrm{H}, \mathrm{dd}, J=5.2$, $10.8 \mathrm{~Hz}, \mathrm{H}-3), 6.18\left(1 \mathrm{H}, \mathrm{t}-\mathrm{like}, J=6.4 \mathrm{~Hz}\right.$, AZT-H-1'), $6.86\left(1 \mathrm{H}, \mathrm{s}, \mathrm{CH}(\mathrm{Ph})_{2}\right), 7.24-7.33$ $\left(11 \mathrm{H}, \mathrm{m}\right.$, aromatic-H and AZT-H-6), $8.59(1 \mathrm{H}, \mathrm{br} \mathrm{s}, \mathrm{AZT}-\mathrm{NH}) ;{ }^{13} \mathrm{C} \mathrm{NMR}\left(\mathrm{CDCl}_{3}, 100\right.$ MHz) $\delta 12.4$ (AZT-CH3), 14.6 (C-27), 14.8 (C-29), 15.9 (C-26), 16.0 (C-25), 16.5 (C-24), 18.1 (C-6), 20.7 (C-11), 21.5 (C-21), 22.9 (C-30), 23.5 (C-2), 25.2, 25.4 (3-Odimethylsuccinyl $\left.\mathrm{CH}_{3}\right), 25.0$, 25.9 (28-O-dimethylsuccinyl $\left.\mathrm{CH}_{3}\right), 26.8(\mathrm{C}-12), 26.8(\mathrm{C}-15)$, 27.9 (C-23), 29.4 (C-20), 29.8 (C-16), 34.1 (C-7), 34.6 (C-22), 36.9 (C-10), 37.1 (C-13), 37.4 (AZT-C-2'), 37.6 (C-4), 38.3 (C-1), 40.7 (dimethylsuccinyl C-3', C-3"), 40.9 (C-8), 42.8 (C-14), 44.5 (3-O-dimethylsuccinyl C-2', C-19), 44.6 (28-O-dimethylsuccinyl C-2"), 46.5 (C-17), 48.1 (C-18), 49.9 (C-9), 55.3 (C-5), 60.4 (AZT-C-3'), 63.1 (C-28), 63.3 (AZTC-5'), $77.0\left(\mathrm{CH}(\mathrm{Ph})_{2}\right), 81.3$ (C-3), 81.9 (AZT-C-4'), 84.8 (AZT-C-1'), 111.4 (AZT-C-5), 127.0, 127.1 (benzhydryl C-2', 6'), 127.7 (benzyhydryl C-4'), 128.4 (benzhydryl C-3', 5'), 135.0 (AZT-C-6), 140.3 (benzhydryl C-1'), 150.0 (AZT-C-2), 163.3 (AZT-C-4), 170.9 (3-Odimethylsuccinyl 1'-COO-), 171.7 (28-O-dimethylsuccinyl 1"-COO-), 175.4 (3-Odimethylsuccinyl 4'-COO-), 176.5 (28-O-dimethylsuccinyl 4"-COO-AZT). HRESIMS (positive) $\mathrm{m} / 2.1116 .6604[\mathrm{M}+\mathrm{H}]^{+}$(calcd for $\mathrm{C}_{65} \mathrm{H}_{90} \mathrm{~N}_{5} \mathrm{O}_{11}, 1116.6637$ ).

Compound 42: $[\alpha]_{\mathrm{D}}^{30}-1.8^{\circ}\left(c 2.69 \mathrm{CHCl}_{3}\right) .{ }^{1} \mathrm{H} \mathrm{NMR}\left(\mathrm{CDCl}_{3}, 400 \mathrm{MHz}\right) \delta 0.77,0.84$ (each $3 \mathrm{H}, \mathrm{d}, J=8.0 \mathrm{~Hz}, \mathrm{CH} 3-29,30), 0.74\left(3 \mathrm{H}, \mathrm{s}, \mathrm{CH}_{3}-24\right), 0.78\left(3 \mathrm{H}, \mathrm{s}, \mathrm{CH}_{3}-23\right), 0.80(3 \mathrm{H}, \mathrm{s}$, $\left.\mathrm{CH}_{3}-25\right), 0.94$ (3H, s, CH3-27), 1.02 (3H, s, $\left.\mathrm{CH}_{3}-26\right), 1.31$ (6H, s, 3-O-dimethylsuccinyl $\mathrm{CH}_{3}$ ), 1.31, 1.34 (each $3 \mathrm{H}, \mathrm{s}, 28$ - $O$-dimethylsuccinyl $\left.\mathrm{CH}_{3}\right), 1.96$ (3H, br s, AZT-CH3), 2.32-2.39, 2.44-2.50 (each 1H, m, AZT- $\mathrm{H}_{2}-2$ '), 2.65, 2.70 (each $1 \mathrm{H}, \mathrm{d}, J=16.0 \mathrm{~Hz}, 3-O-$ dimethylsuccinyl $\left.\mathrm{H}_{2}-2^{\prime}\right), 2.61,2.66$ (each $1 \mathrm{H}, \mathrm{d}, J=16.0 \mathrm{~Hz}, 28$ - $O$-dimethylsuccinyl $\mathrm{H}_{2}-3$ "), 3.81, 4.29 (each 1H, d, $\left.J=10.8 \mathrm{~Hz}, \mathrm{H}_{2}-28\right), 4.04(1 \mathrm{H}, \mathrm{q}-\mathrm{like}$, AZT-H-4'), 4.18-4.22 (1H, m, AZT-H-3'), $4.26(1 \mathrm{H}, \mathrm{dd}, J=3.6,12.4 \mathrm{~Hz}$, AZT-H-5a'), $4.42(1 \mathrm{H}, \mathrm{dd}, J=4.4,12.4 \mathrm{~Hz}$, AZT-H-5b'), 4.44 (1H, dd, $J=5.2,10.8 \mathrm{~Hz}, \mathrm{H}-3), 6.15$ (1H, t-like, $J=6.4 \mathrm{~Hz}$, AZT-H-1'), $6.86\left(1 \mathrm{H}, \mathrm{s}, \mathrm{CH}(\mathrm{Ph})_{2}\right), 7.24-7.33(11 \mathrm{H}, \mathrm{m}$, aromatic-H and AZT-H-6), $8.93(1 \mathrm{H}, \mathrm{br}$ s, AZT$\mathrm{NH}) ;{ }^{13} \mathrm{C}$ NMR (CDCl3, $\left.100 \mathrm{MHz}\right) \delta 12.5$ (AZT-CH3), 14.6 (C-27), 14.9 (C-29), 16.0 (C-26, 25), 16.5 (C-24), 18.1 (C-6), 20.7 (C-11), 21.5 (C-21), 22.9 (C-30), 23.5 (C-2), 25.2, 25.4 (3-O-dimethylsuccinyl $\left.\mathrm{CH}_{3}\right), 25.4,25.8$ (28-O-dimethylsuccinyl $\left.\mathrm{CH}_{3}\right), 26.8(\mathrm{C}-12)$, 26.9 (C-15), 27.9 (C-23), 29.4 (C-20), 29.9 (C-16), 34.1 (C-7), 34.6 (C-22), 36.9 (C-10), 37.1 (C-13), 37.5 (AZT-C-2', 37.6 (C-4), 38.3 (C-1), 40.7 (3-O-dimethylsuccinyl C-3'), 40.9 (C-8), 41.0 (28-O-dimethylsuccinyl C-2"), 42.8 (C-14), 43.8 (28-O-dimethylsuccinyl C-3"), 44.5 (3-O-dimethylsuccinyl C-2', C-19), 46.7 (C-17), 48.1 (C-18), 49.9 (C-9), 55.3 (C-5), 60.2 (AZT-C-3'), 62.8 (AZT-C-5'), 63.3 (C-28), $77.0\left(\mathrm{CH}(\mathrm{Ph})_{2}\right), 81.3$ (C-3), 81.7 (AZTC-4'), 85.1 (AZT-C-1'), 111.3 (AZT-C-5), 127.0, 127.1 (benzhydryl C-2', 6'), 127.7 (benzyhydryl C-4'), 128.4 (benzhydryl C-3', 5'), 135.3 (AZT-C-6), 140.3 (benzhydryl C-1'), 150.0 (AZT-C-2), 163.5 (AZT-C-4), 170.7 (28-O-dimethylsuccinyl 4"-COO-AZT), 170.8 (3-O-dimethylsuccinyl 1'-COO-), 175.4 (3-O-dimethylsuccinyl 4'-COO-), 176.9 (28-Odimethylsuccinyl 1"-COO-). HRESIMS (positive) $\mathrm{m} / z$ $1138.6445[\mathrm{M}+\mathrm{Na}]^{+}$(calcd for $\left.\mathrm{C}_{65} \mathrm{H}_{89} \mathrm{~N}_{5} \mathrm{O}_{11} \mathrm{Na}, 1138.6456\right)$.

3'-Azido-3'-deoxythymidine-5'-yl 3-O-4'-benzhydryloxy-3',3'-dimethylsuccinyl)-lup-28yl 3,3-dimethylglutarate (43): Yield $66.1 \%$ (starting from $118 \mathrm{mg}$ of 29); white solid. [ $\alpha]_{\mathrm{D}}^{26}$ $-0.32^{\circ}\left(c 1.53 \mathrm{CHCl}_{3}\right) .{ }^{1} \mathrm{H} \mathrm{NMR}\left(\mathrm{CDCl}_{3}, 400 \mathrm{MHz}\right) \delta 0.76,0.83($ each $3 \mathrm{H}, \mathrm{d}, J=8.0 \mathrm{~Hz}$, $\left.\mathrm{CH}_{3}-29,30\right), 0.73\left(3 \mathrm{H}, \mathrm{s}, \mathrm{CH}_{3}-24\right), 0.77$ (3H, s, $\left.\mathrm{CH} 3-23\right), 0.78$ (3H, s, $\left.\mathrm{CH}_{3}-25\right), 0.93(3 \mathrm{H}$, $\mathrm{s}, \mathrm{CH}_{3}$-27), 1.00 (3H, s, $\mathrm{CH}_{3}-26$ ), 1.11, 1.14 (each $3 \mathrm{H}, \mathrm{s}, 28$-O-dimethylglutaryl $\mathrm{CH}_{3}$ ), 1.29 , 1.30 (each $3 \mathrm{H}, \mathrm{s}, 3-\mathrm{O}$-dimethylsuccinyl $\left.\mathrm{CH}_{3}\right), 1.94\left(3 \mathrm{H}, \mathrm{br}\right.$ s, AZT- $\left.\mathrm{CH}_{3}\right), 2.31-2.38,2.43-$ $2.50\left(2 \mathrm{H}, \mathrm{m}, \mathrm{AZT}-\mathrm{H}_{2}-2^{\prime}\right), 2.41,2.48$ (each $1 \mathrm{H}, \mathrm{d}, J=14.4 \mathrm{~Hz}, 28$ - $O$-dimethylglutaryl $\mathrm{H}_{2}-2$ "), 2.47, 2.55 (each $1 \mathrm{H}, \mathrm{d}, J=15.2 \mathrm{~Hz}, 28$ - $O$-dimethylglutaryl $\left.\mathrm{H}_{2}-4 "\right)$, 2.63, 2.68 (each $1 \mathrm{H}, \mathrm{d}, J=16.0 \mathrm{~Hz}, 3-O$-dimethylsuccinyl $\mathrm{H}_{2}-2$ '), 3.77, 4.24 (each $1 \mathrm{H}, \mathrm{d}, J=10.8 \mathrm{~Hz}$, 
$\mathrm{H}_{2}$-28), 4.05 (1H, q-like, AZT-H-4'), 4.19-4.23 (1H, m, AZT-H-3'), 4.25 (1H, dd, $J=4.4$, $12.0 \mathrm{~Hz}$, AZT-H-5a'), $4.43\left(1 \mathrm{H}, \mathrm{dd}, J=4.4,12.0 \mathrm{~Hz}, \mathrm{AZT}-\mathrm{H}-5 \mathrm{~b}^{\prime}\right), 4.43(1 \mathrm{H}, \mathrm{dd}, J=4.4$, $12.0 \mathrm{~Hz}, \mathrm{H}-3), 6.14\left(1 \mathrm{H}, \mathrm{t}-\mathrm{like}, J=6.4 \mathrm{~Hz}\right.$, AZT-H-1'), $6.85\left(1 \mathrm{H}, \mathrm{s}, \mathrm{CH}(\mathrm{Ph})_{2}\right), 7.23-7.31$ $\left(11 \mathrm{H}, \mathrm{m}\right.$, aromatic-H and AZT-H-6), $8.48(1 \mathrm{H}, \mathrm{br} \mathrm{s}$, AZT-NH $) ;{ }^{13} \mathrm{C} \mathrm{NMR}\left(\mathrm{CDCl}_{3}, 100\right.$ MHz) $\delta 12.6\left(\mathrm{AZT}^{-\mathrm{CH}_{3}}\right), 14.6(\mathrm{C}-27), 14.9(\mathrm{C}-29), 16.0$ (C-26, 25), 16.5 (C-24), 18.1 (C-6), 20.8 (C-11), 21.6 (C-21), 22.9 (C-30), 23.5 (C-2), 25.2, 25.4 (3-O-dimethylsuccinyl $\mathrm{CH}_{3}$ ), 26.8 (C-12), 26.9 (C-15), 27.9 (28-O-dimethylglutaryl $\mathrm{CH}_{3}$ ), 28.0 (C-23), 29.4 (C-20), 29.9 (C-16), 32.6 (28-O-dimethylglutaryl C-3"), 34.2(C-7), 34.7 (C-22), 37.0 (C-10), 37.1 (C-13), 37.5 (AZT-C-2'), 37.7 (C-4), 38.3 (C-1), 40.7 (3-O-dimethylsuccinyl C-3'), 40.9 (C-8), 42.8 (C-14), 44.5 (3-O-dimethylsuccinyl C-2', 28-O-dimethylglutaryl C-4", C-19), 45.0 (28-O-dimethylsuccinyl C-2"), 46.4 (C-17), 48.1 (C-18), 49.9 (C-9), 55.3 (C-5), 60.4 (AZT-C-3'), 62.7 (C-28, AZT-C-5'), $77.0\left(\mathrm{CH}(\mathrm{Ph})_{2}\right), 81.3$ (C-3), 81.8 (AZT-C-4'), 85.2 (AZT-C-1'), 111.3 (AZT-C-5), 127.0, 127.1 (benzhydryl C-2', 6'), 127.7 (benzyhydryl C-4'), 128.4 (benzhydryl C-3', 5'), 135.2 (AZT-C-6), 140.3 (benzhydryl C-1'), 149.9 (AZT-C-2), 163.2 (AZT-C-4), 170.8 (3-O-dimethylsuccinyl 1'-COO-), 171.2 (28-O-dimethylglutaryl 5"-COO-AZT), 172.3 (28-O-dimethylglutaryl 1"-COO-), 175.4 (3-O-dimethylsuccinyl 4'COO-). HRESIMS (positive) $\mathrm{m} / z 1130.6781[\mathrm{M}+\mathrm{H}]^{+}$(calcd for $\mathrm{C}_{66} \mathrm{H}_{92} \mathrm{~N}_{5} \mathrm{O}_{11}, 1130.6793$ ).

1-(3'-Azido-3'-deoxythymidine-5'-yl)-4-[3-O-(4'-benzhydryloxyglutaryl)-lup-28-yl] 2,2dimethylsuccinate (44) and 4-(3'-Azido-3'-deoxythymidine-5'-yl)-1-[3-O-(4'benzhydryloxyglutaryl)-lup-28-yl] 2,2-dimethylsuccinate (45): Yield 15.8\% and 10.5\% respectively (starting from $208 \mathrm{mg}$ of mixture of $\mathbf{3 0}$ and 31); separated by preparative HPLC (cholester, $\mathrm{MeOH} / \mathrm{H}_{2} \mathrm{O}=97: 3$ ); colorless oil.

Compound 44: $[\alpha]_{\mathrm{n}}^{27}-1.96^{\circ}\left(c 0.72 \mathrm{CHCl}_{3}\right) .{ }^{1} \mathrm{H} \mathrm{NMR}\left(\mathrm{CDCl}_{3}, 400 \mathrm{MHz}\right) \delta 0.77,0.85$ (each $\left.3 \mathrm{H}, \mathrm{d}, J=8.0 \mathrm{~Hz}, \mathrm{CH}_{3}-29,30\right), 0.83\left(3 \mathrm{H}, \mathrm{s}, \mathrm{CH}_{3}-24\right), 0.84\left(3 \mathrm{H}, \mathrm{s}, \mathrm{CH}_{3}-23\right), 0.86(3 \mathrm{H}, \mathrm{s}$, $\left.\mathrm{CH}_{3}-25\right), 0.96$ (3H, s, $\left.\mathrm{CH}_{3}-27\right), 1.03$ (3H, s, $\left.\mathrm{CH}_{3}-26\right), 1.31,1.32$ (each $3 \mathrm{H}, \mathrm{s}, 28-O-$ dimethylsuccinyl $\left.\mathrm{CH}_{3}\right), 1.94\left(3 \mathrm{H}\right.$, br s, AZT- $\left.\mathrm{CH}_{3}\right), 2.00(2 \mathrm{H}$, quint, $J=7.2 \mathrm{~Hz}, 3-O$-glutaryl $\left.\mathrm{H}_{2}-3^{\prime}\right), 2.26-2.35,2.44-2.50$ (each $\left.1 \mathrm{H}, \mathrm{m}, \mathrm{AZT}-\mathrm{H}_{2}-2^{\prime}\right), 2.35(2 \mathrm{H}, \mathrm{t}, J=7.2 \mathrm{~Hz}, 3-O$-glutaryl $\left.\mathrm{H}_{2}-2^{\prime}\right), 2.50\left(2 \mathrm{H}, \mathrm{t}, J=7.2 \mathrm{~Hz}, 3-O\right.$-glutaryl $\left.\mathrm{H}_{2}-4^{\prime}\right), 2.60,2.74$ (each $1 \mathrm{H}, \mathrm{d}, J=16.0 \mathrm{~Hz}, 28$ $O$-dimethylsuccinyl $\mathrm{H}_{2}-2$ "), 3.82, 4.28 (each $\left.1 \mathrm{H}, \mathrm{d}, J=10.8 \mathrm{~Hz}, \mathrm{H}_{2}-28\right), 4.09$ (1H, q-like, AZT-H-4'), 4.25-4.30 (1H, m, AZT-H-3'), 4.28 (1H, dd, $J=4.0,12.0 \mathrm{~Hz}$, AZT-H-5a'), 4.51 $(1 \mathrm{H}, \mathrm{dd}, J=4.0,12.0 \mathrm{~Hz}$, AZT-H-5b'), $4.49(1 \mathrm{H}, \mathrm{dd}, J=6.0,10.0 \mathrm{~Hz}, \mathrm{H}-3), 6.17(1 \mathrm{H}$, t-like, $\left.J=6.4 \mathrm{~Hz}, \mathrm{AZT}-\mathrm{H}-1^{\prime}\right), 6.89\left(1 \mathrm{H}, \mathrm{s}, \mathrm{CH}(\mathrm{Ph})_{2}\right), 7.25-7.34(11 \mathrm{H}, \mathrm{m}$, aromatic-H and AZT$\mathrm{H}-6), 8.71\left(1 \mathrm{H}, \mathrm{br}\right.$ s, AZT-NH); ${ }^{13} \mathrm{C}$ NMR $\left(\mathrm{CDCl}_{3}, 100 \mathrm{MHz}\right) \delta 12.4\left(\mathrm{AZT}-\mathrm{CH}_{3}\right), 14.6$ (C-27), 14.9 (C-29), 16.0 (C-26), 16.1 (C-25), 16.5 (C-24), 18.2 (C-6), 20.4 (3-O-glutaryl C-3'), 20.8 (C-11), 21.6 (C-21), 22.9 (C-30), 23.7 (C-2), 25.1, 25.8 (28-O-dimethylsuccinyl $\mathrm{CH}_{3}$ ), 26.8 (C-12), 26.9 (C-15), 28.0 (C-23), 29.4 (C-20), 29.9 (C-16), 33.6 (3-O-glutaryl C-4'), 33.7 (3-O-glutaryl C-2'), 34.2 (C-7), 34.6 (C-22), 37.0 (C-10), 37.2 (C-13), 37.5 (AZT-C-2'), 37.8 (C-4), 38.4 (C-1), 40.7 (28-O-dimethylsuccinyl C-3"), 40.9 (C-8), 42.9 (C-14), 44.5 (C-19), 44.6 (28-O-dimethylsuccinyl C-2"), 46.5 (C-17), 48.1 (C-18), 49.9 (C-9), 55.3 (C-5), 60.4 (AZT-C-3'), 63.1 (C-28), 63.3 (AZT-C-5'), $76.9\left(\mathrm{CH}(\mathrm{Ph})_{2}\right), 81.0$ (C-3), 82.0 (AZT-C-4'), 84.9 (AZT-C-1'), 111.4 (AZT-C-5), 127.0 (benzhydryl C-2', 6'), 127.9 (benzyhydryl C-4'), 128.5 (benzhydryl C-3', 5'), 135.0 (AZT-C-6), 140.2 (benzhydryl C-1'), 150.0 (AZT-C-2), 163.4 (AZT-C-4), 171.7 (28-O-dimethylsuccinyl 1"-COO-), 171.9 (3-O-glutaryl 5'-COO-), 172.6 (3-O-glutaryl 1'-COO-), 176.5 (28-O-dimethylsuccinyl 4"COO-AZT). HRESIMS (positive) $\mathrm{m} / 21102.6483[\mathrm{M}+\mathrm{H}]^{+}$(calcd for $\mathrm{C}_{64} \mathrm{H}_{88} \mathrm{~N}_{5} \mathrm{O}_{11}$, 1102.6480).

Compound 45: $[\alpha]_{\mathrm{D}}^{27}+1.4^{\circ}\left(c 0.99 \mathrm{CHCl}_{3}\right) .{ }^{1} \mathrm{H} \mathrm{NMR}\left(\mathrm{CDCl}_{3}, 400 \mathrm{MHz}\right) \delta 0.77,0.85$ (each $\left.3 \mathrm{H}, \mathrm{d}, J=8.0 \mathrm{~Hz}, \mathrm{CH}_{3}-29,30\right), 0.83\left(3 \mathrm{H}, \mathrm{s}, \mathrm{CH}_{3}-24\right), 0.84\left(3 \mathrm{H}, \mathrm{s}, \mathrm{CH}_{3}-23\right), 0.86(3 \mathrm{H}, \mathrm{s}$, $\left.\mathrm{CH}_{3}-25\right), 0.96$ (3H, s, $\left.\mathrm{CH}_{3}-27\right), 1.05$ (3H, s, $\left.\mathrm{CH}_{3}-26\right), 1.31,1.34$ (each $3 \mathrm{H}, \mathrm{s}, 28-O-$ 
dimethylsuccinyl $\left.\mathrm{CH}_{3}\right), 1.96\left(3 \mathrm{H}\right.$, br s, AZT- $\left.\mathrm{CH}_{3}\right), 2.00(2 \mathrm{H}$, quint, $J=7.2 \mathrm{~Hz}, 3-O$-glutaryl $\left.\mathrm{H}_{2}-3^{\prime}\right), 2.33-2.52\left(2 \mathrm{H}, \mathrm{m}\right.$, AZT-H $\left.{ }_{2} 2^{\prime}\right), 2.35\left(2 \mathrm{H}, \mathrm{t}, J=7.2 \mathrm{~Hz}, 3-O\right.$-glutaryl $\left.\mathrm{H}_{2}-2^{\prime}\right), 2.50(2 \mathrm{H}$, t, $J=7.2 \mathrm{~Hz}, 3-O$-glutaryl $\mathrm{H}_{2}-4$ '), 2.62, 2.66 (each $1 \mathrm{H}, \mathrm{d}, J=16.0 \mathrm{~Hz}, 28-O$ dimethylsuccinyl $\mathrm{H}_{2}-2$ "), 3.82, 4.30 (each $1 \mathrm{H}, \mathrm{d}, J=10.8 \mathrm{~Hz}, \mathrm{H}_{2}-28$ ), 4.05 (1H, q-like, AZT-H-4'), 4.17-4.22 (1H, m, AZT-H-3'), $4.26(1 \mathrm{H}, \mathrm{dd}, J=3.6,12.0 \mathrm{~Hz}$, AZT-H-5a'), 4.42 $(1 \mathrm{H}, \mathrm{dd}, J=4.0,12.0 \mathrm{~Hz}$, AZT-H-5b'), $4.49(1 \mathrm{H}, \mathrm{dd}, J=6.0,10.0 \mathrm{~Hz}, \mathrm{H}-3), 6.15(1 \mathrm{H}, \mathrm{t}-\mathrm{like}$, $\left.J=6.4 \mathrm{~Hz}, \mathrm{AZT}-\mathrm{H}-1^{\prime}\right), 6.89\left(1 \mathrm{H}, \mathrm{s}, \mathrm{CH}(\mathrm{Ph})_{2}\right), 7.25-7.34(11 \mathrm{H}, \mathrm{m}$, aromatic-H and AZT$\mathrm{H}-6), 8.74\left(1 \mathrm{H}, \mathrm{br}\right.$ s, AZT-NH); ${ }^{13} \mathrm{C}$ NMR $\left(\mathrm{CDCl}_{3}, 100 \mathrm{MHz}\right) \delta 12.5\left(\mathrm{AZT}-\mathrm{CH}_{3}\right), 14.6$ (C-27), 14.9 (C-29), 16.0 (C-26), 16.1 (C-25), 16.5 (C-24), 18.1 (C-6), 20.4 (3-O-glutaryl C-3'), 20.8 (C-11), 21.6 (C-21), 22.9 (C-30), 23.7 (C-2), 25.5, 25.8 (28-O-dimethylsuccinyl $\mathrm{CH}_{3}$ ), 26.8 (C-12), 26.9 (C-15), 28.0 (C-23), 29.4 (C-20), 29.9 (C-16), 33.6 (3-O-glutaryl C-4'), 33.7 (3-O-glutaryl C-2'), 34.2 (C-7), 34.7 (C-22), 37.0 (C-10), 37.2 (C-13), 37.5 (AZT-C-2'), 37.8 (C-4), 38.4 (C-1), 40.9 (C-8), 41.0 (28-O-dimethylsuccinyl C-2"), 42.9 (C-14), 43.8 (28-O-dimethylsuccinyl C-3"), 44.6 (C-19), 46.7 (C-17), 48.2 (C-18), 50.0 (C-9), 55.3 (C-5), 60.3 (AZT-C-3'), 62.9 (AZT-C-5'), $63.3(\mathrm{C}-28), 76.9\left(\mathrm{CH}(\mathrm{Ph})_{2}\right), 81.0$ (C-3), 81.8 (AZT-C-4'), 85.2 (AZT-C-1'), 111.3 (AZT-C-5), 127.0 (benzhydryl C-2', 6'), 127.9 (benzyhydryl C-4'), 128.5 (benzhydryl C-3', 5'), 135.3 (AZT-C-6), 140.2 (benzhydryl C-1'), 150.0 (AZT-C-2), 163.4 (AZT-C-4), 170.7 (28-O-dimethylsuccinyl 4"-COO-AZT), 171.9 (3-O-glutaryl 5'-COO-), 172.6 (3-O-glutaryl 1'-COO-), 176.8 (28-Odimethylsuccinyl 1"-COO-). HRESIMS (positive) $\mathrm{m} / \mathrm{z} 1102.6475[\mathrm{M}+\mathrm{H}]^{+}$(calcd for $\mathrm{C}_{64} \mathrm{H}_{88} \mathrm{~N}_{5} \mathrm{O}_{11}, 1102.6480$ ).

\section{3'-Azido-3'-deoxythymidine-5'-yl 3-O-(4'-benzhydryloxyglutaryl)-lup-28-yl}

glutarate(46): Yield $49.8 \%$ (starting from $79 \mathrm{mg}$ of 32); colorless oil. $[\alpha]_{\mathrm{D}}^{27}+4.4^{\circ}(c 0.93$, $\left.\mathrm{CHCl}_{3}\right) .{ }^{1} \mathrm{H} \mathrm{NMR}\left(\mathrm{CDCl}_{3}, 400 \mathrm{MHz}\right) \delta 0.78,0.84$ (each $\left.3 \mathrm{H}, \mathrm{d}, J=8.0 \mathrm{~Hz}, \mathrm{CH}_{3}-29,30\right), 0.83$ (each $\left.3 \mathrm{H}, \mathrm{s}, \mathrm{CH}_{3}-24, \mathrm{CH}_{3}-23\right), 0.86\left(3 \mathrm{H}, \mathrm{s}, \mathrm{CH}_{3}-25\right), 0.96\left(3 \mathrm{H}, \mathrm{s}, \mathrm{CH}_{3}-27\right), 1.05(3 \mathrm{H}, \mathrm{s}$, $\left.\mathrm{CH}_{3}-26\right), 1.94(3 \mathrm{H}, \mathrm{d}, J=1.2 \mathrm{~Hz}, \mathrm{AZT}-\mathrm{CH} 3), 1.99(4 \mathrm{H}$, quint, $J=7.2 \mathrm{~Hz}, 3-O, 28-O-$ glutaryl $\left.\mathrm{H}_{2}-3^{\prime}\right), 2.32-2.52\left(2 \mathrm{H}, \mathrm{m}\right.$, AZT- $\left.\mathrm{H}_{2}-2^{\prime}\right), 2.35\left(2 \mathrm{H}, \mathrm{t}, J=7.2 \mathrm{~Hz}, 3-O\right.$-glutaryl $\left.\mathrm{H}_{2}-2^{\prime}\right)$, $2.41\left(2 \mathrm{H}, \mathrm{t}, J=7.2 \mathrm{~Hz}, 28-O\right.$-glutaryl $\left.\mathrm{H}_{2}-2 "\right), 2.46\left(2 \mathrm{H}, \mathrm{t}, J=7.2 \mathrm{~Hz}, 28\right.$ - $O$-glutaryl $\left.\mathrm{H}_{2}-4 "\right)$, $2.50\left(2 \mathrm{H}, \mathrm{t}, J=7.2 \mathrm{~Hz}, 3-O\right.$-glutaryl $\left.\mathrm{H}_{2}-4^{\prime}\right), 3.83,4.30$ (each $1 \mathrm{H}, \mathrm{d}, J=11.2 \mathrm{~Hz}, \mathrm{H}_{2}-28$ ), 4.07 (1H, q-like, AZT-H-4'), 4.20-4.24 (1H, m, AZT-H-3'), $4.32(1 \mathrm{H}, \mathrm{dd}, J=4.0,12.0 \mathrm{~Hz}$, AZT-H-5a'), $4.40(1 \mathrm{H}, \mathrm{dd}, J=4.4,12.0 \mathrm{~Hz}$, AZT-H-5b'), $4.49(1 \mathrm{H}, \mathrm{dd}, J=6.0,10.4 \mathrm{~Hz}$, H-3), $6.10\left(1 \mathrm{H}, \mathrm{t}\right.$-like, $J=6.4 \mathrm{~Hz}$, AZT-H-1'), $6.89\left(1 \mathrm{H}, \mathrm{s}, \mathrm{CH}(\mathrm{Ph})_{2}\right), 7.25-7.33(11 \mathrm{H}, \mathrm{m}$, aromatic-H and AZT-H-6), $9.04\left(1 \mathrm{H}, \mathrm{br}\right.$ s, AZT-NH); ${ }^{13} \mathrm{C} \mathrm{NMR}\left(\mathrm{CDCl}_{3}, 100 \mathrm{MHz}\right) \delta 12.5$ $\left(\mathrm{AZT}-\mathrm{CH}_{3}\right), 14.6$ (C-27), 14.8 (C-29), 16.0 (C-26, -25), 16.5 (C-24), 18.1 (C-6), 20.0 (28$O$-glutaryl C-3"), 20.3 (3-O-glutaryl C-3'), 20.8 (C-11), 21.6 (C-21), 22.8 (C-30), 23.7 (C-2), 26.8 (C-12), 26.9 (C-15), 28.0 (C-23), 29.4 (C-20), 29.9 (C-16), 33.1 (28-O-glutaryl C-4"), 33.2 (28-O-glutaryl C-2"), 33.6 (3-O-glutaryl C-4'), 33.7 (3-O-glutaryl C-2'), 34.2 (C-7), 34.6 (C-22), 37.0 (C-10), 37.2 (C-13), 37.4 (AZT-C-2'), 37.8 (C-4), 38.3 (C-1), 40.9 (C-8), 42.8 (C-14), 44.5 (C-19), 46.5 (C-17), 48.1 (C-18), 49.9 (C-9), 55.3 (C-5), 60.6 (AZT-C-3'), 62.9 (C-28), 63.3 (AZT-C-5'), $76.8\left(\mathrm{CH}(\mathrm{Ph})_{2}\right), 81.0$ (C-3), 81.7 (AZT-C-4'), 85.6 (AZTC-1'), 111.3 (AZT-C-5), 127.0 (benzhydryl C-2', 6'), 127.8 (benzyhydryl C-4'), 128.4 (benzhydryl C-3', 5'), 135.3 (AZT-C-6), 140.2 (benzhydryl C-1'), 150.0 (AZT-C-2), 163.5 (AZT-C-4), 171.8 (3-O-glutaryl 5'-COO-), 172.3 (28-O-glutaryl 5"-COO-AZT), 172.6 (3$O$-glutaryl 1'-COO-), 173.1 (28-O-glutaryl 1"-COO-). HRESIMS (positive) $\mathrm{m} / z 1112.6271$ $[\mathrm{M}+\mathrm{Na}]^{+}$(calcd for $\mathrm{C}_{63} \mathrm{H}_{87} \mathrm{~N}_{5} \mathrm{O}_{11} \mathrm{Na}, 1112.6300$ ).

\section{3'-Azido-3'-deoxythymidine-5'-yl 3-O-(4'-benzhydryloxyglutaryl)-lup-28-yl 3,3-}

dimethylglutarate (47): Yield $48.7 \%$ (starting from $76 \mathrm{mg}$ of 33); colorless oil. $[\alpha]_{\mathrm{D}}^{27}+4.1^{\circ}$ (c $\left.0.54, \mathrm{CHCl}_{3}\right) .{ }^{1} \mathrm{H} \mathrm{NMR}\left(\mathrm{CDCl}_{3}, 400 \mathrm{MHz}\right) \delta 0.78,0.84$ (each $3 \mathrm{H}, \mathrm{d}, J=8.0 \mathrm{~Hz}, \mathrm{CH}_{3}-29$, $30), 0.83\left(3 \mathrm{H}, \mathrm{s}, \mathrm{CH}_{3}-24\right), 0.84\left(3 \mathrm{H}, \mathrm{s}, \mathrm{CH}_{3}-23\right), 0.86\left(3 \mathrm{H}, \mathrm{s}, \mathrm{CH}_{3}-25\right), 0.96\left(3 \mathrm{H}, \mathrm{s}, \mathrm{CH}_{3}-27\right)$, $1.04\left(3 \mathrm{H}, \mathrm{s}, \mathrm{CH}_{3}-26\right), 1.12,1.15$ (each $3 \mathrm{H}, \mathrm{s}, 28$ - $O$-dimethylglutaryl $\left.\mathrm{CH}_{3}\right), 1.95(3 \mathrm{H}, \mathrm{d}, J=$ 
$\left.1.2 \mathrm{~Hz}, \mathrm{AZT}-\mathrm{CH}_{3}\right), 2.00\left(2 \mathrm{H}\right.$, quint, $J=7.2 \mathrm{~Hz}, 3-O$-glutaryl $\left.\mathrm{H}_{2}-3^{\prime}\right), 2.32-2.52(2 \mathrm{H}, \mathrm{m}$, AZT- $\left.\mathrm{H}_{2}-2^{\prime}\right), 2.35\left(2 \mathrm{H}, \mathrm{t}, J=7.2 \mathrm{~Hz}, 3-O\right.$-glutaryl $\left.\mathrm{H}_{2}-2^{\prime}\right), 2.43,2.49$ (each $1 \mathrm{H}, \mathrm{d}, J=14.4$ $\mathrm{Hz}, 28$ - $O$-dimethylglutaryl $\mathrm{H}_{2}-2 "$ ), 2.48, 2.56 (each $1 \mathrm{H}, \mathrm{d}, J=14.4 \mathrm{~Hz}, 28-O$ -

dimethylglutaryl $\left.\mathrm{H}_{2}-4^{\prime \prime}\right), 2.50\left(2 \mathrm{H}, \mathrm{t}, J=7.2 \mathrm{~Hz}, 3-O\right.$-glutaryl $\left.\mathrm{H}_{2}-4^{\prime}\right), 3.79,4.27$ (each $1 \mathrm{H}, \mathrm{d}$, $\left.J=11.2 \mathrm{~Hz}, \mathrm{H}_{2}-28\right), 4.07$ (1H, q-like, AZT-H-4'), 4.20-4.28 (1H, m, AZT-H-3'), $4.26(1 \mathrm{H}$, $\mathrm{dd}, J=3.6,12.0 \mathrm{~Hz}$, AZT-H-5a'), $4.43(1 \mathrm{H}, \mathrm{dd}, J=4.4,12.0 \mathrm{~Hz}$, AZT-H-5b'), 4.49 (1H, dd, $J=6.0,10.4 \mathrm{~Hz}, \mathrm{H}-3), 6.15\left(1 \mathrm{H}, \mathrm{t}-\mathrm{like}, J=6.4 \mathrm{~Hz}, \mathrm{AZT}-\mathrm{H}-1^{\prime}\right), 6.89\left(1 \mathrm{H}, \mathrm{s}, \mathrm{CH}(\mathrm{Ph})_{2}\right), 7.25-$ $7.33\left(11 \mathrm{H}, \mathrm{m}\right.$, aromatic-H and AZT-H-6), $8.91(1 \mathrm{H}, \mathrm{br} \mathrm{s}, \mathrm{AZT}-\mathrm{NH}) ;{ }^{13} \mathrm{C} \mathrm{NMR}\left(\mathrm{CDCl}_{3}, 100\right.$ $\mathrm{MHz}) \delta 12.5\left(\mathrm{AZT}-\mathrm{CH}_{3}\right), 14.6$ (C-27), 14.8 (C-29), 16.0 (C-26, -25), 16.5 (C-24), 18.1 (C-6), 20.3 (3-O-glutaryl C-3'), 20.8 (C-11), 21.6 (C-21), 22.8 (C-30), 23.7 (C-2), 26.8 (C-12), 26.9 (C-15), 27.8, 27.9 (28-O-dimethylglutaryl $\mathrm{CH}_{3}$ ), 28.0 (C-23), 29.4 (C-20), 29.9 (C-16), 32.5 (28- $O$-dimethylglutaryl C-3"), 33.6 (3- $O$-glutaryl C-4'), 33.7 (3-O-glutaryl C-2'), 34.2 (C-7), 34.7 (C-22), 37.0 (C-10), 37.2 (C-13), 37.5 (AZT-C-2'), 37.8 (C-4), 38.3 (C-1), 40.9 (C-8), 42.8 (C-14), 44.5 (28-O-dimethylglutaryl C-4" and C-19), 45.1 (28-Odimethylglutaryl C-2"), 46.4 (C-17), 48.1 (C-18), 49.9 (C-9), 55.3 (C-5), 60.5 (AZT-C-3'), $62.7\left(\mathrm{C}-28\right.$, and AZT-C-5'), $76.9\left(\mathrm{CH}(\mathrm{Ph})_{2}\right), 81.0(\mathrm{C}-3), 81.8$ (AZT-C-4'), 85.2 (AZT-C-1'), 111.3 (AZT-C-5), 127.0 (benzhydryl C-2', 6'), 127.9 (benzyhydryl C-4'), 128.5 (benzhydryl C-3', 5'), 135.2 (AZT-C-6), 140.2 (benzhydryl C-1'), 150.1 (AZT-C-2), 163.5 (AZT-C-4), 171.1 (28-O-dimethylglutaryl 5"-COO-AZT), 171.8 (3-O-glutaryl 5'-COO-), 172.2 (28-Odimethylglutaryl 1"-COO-), 172.6 (3-O-glutaryl 1'-COO-). HRESIMS (positive) $\mathrm{m} / \mathrm{z}$ $1138.6449[\mathrm{M}+\mathrm{Na}]^{+}$(calcd for $\mathrm{C}_{65} \mathrm{H}_{89} \mathrm{~N}_{5} \mathrm{O}_{11} \mathrm{Na}, 1138.6456$ ).

3'-Azido-3'-deoxythymidine-5'-yl 3-O-(4'-benzhydryloxy-3',3'-dimethylglutaryl)lup-28-yl 3,3-dimethylglutarate (48): Yield 68.3\% (starting from $126 \mathrm{mg}$ of 34); colorless oil. $[\alpha]_{\mathrm{D}}^{26}+0.97^{\circ}\left(c 2.37, \mathrm{CHCl}_{3}\right) .{ }^{1} \mathrm{H} \mathrm{NMR}\left(\mathrm{CDCl}_{3}, 400 \mathrm{MHz}\right) \delta 0.78,0.84$ (each $3 \mathrm{H}, \mathrm{d}, J=$ $\left.8.0 \mathrm{~Hz}, \mathrm{CH}_{3}-29,30\right), 0.81\left(3 \mathrm{H}, \mathrm{s}, \mathrm{CH}_{3}-24\right), 0.83\left(6 \mathrm{H}, \mathrm{s}, \mathrm{CH}_{3}-23, \mathrm{CH}_{3}-25\right), 0.95(3 \mathrm{H}, \mathrm{s}$, $\left.\mathrm{CH}_{3}-27\right), 1.03\left(3 \mathrm{H}, \mathrm{s}, \mathrm{CH}_{3}-26\right), 1.09\left(6 \mathrm{H}, \mathrm{s}, 3\right.$ - $O$-dimethylglutaryl $\left.\mathrm{CH}_{3}\right), 1.12,1.15$ (each $3 \mathrm{H}, \mathrm{s}, 28-\mathrm{O}$-dimethylglutaryl $\left.\mathrm{CH}_{3}\right), 1.95\left(3 \mathrm{H}, \mathrm{br} \mathrm{s}, \mathrm{AZT}-\mathrm{CH}_{3}\right), 2.32-2.52(2 \mathrm{H}, \mathrm{m}, \mathrm{AZT}-$ $\mathrm{H}_{2}-2^{\prime}$ ), 2.35, 2.42 (each $1 \mathrm{H}, \mathrm{d}, J=14.4 \mathrm{~Hz}, 3-O$-dimethylglutaryl $\mathrm{H}_{2}-2^{\prime}$ ), 2.43, 2.49 (each $1 \mathrm{H}, \mathrm{d}, J=14.4 \mathrm{~Hz}, 28-O$-dimethylglutaryl $\mathrm{H}_{2}-2 "$ ), $2.48,2.56$ (each $1 \mathrm{H}, \mathrm{d}, J=14.4 \mathrm{~Hz}, 28$ $O$-dimethylglutaryl $\mathrm{H}_{2}-4 "$ ), 2.55, 2.58 (each $1 \mathrm{H}, \mathrm{d}, J=14.4 \mathrm{~Hz}, 3$ - $O$-dimethylglutaryl $\mathrm{H}_{2}-4^{\prime}$ ), 3.79, 4.26 (each 1H, d, $\left.J=11.2 \mathrm{~Hz}, \mathrm{H}_{2}-28\right), 4.07$ (1H, q-like, AZT-H-4'), 4.21-4.28 $(1 \mathrm{H}, \mathrm{m}$, AZT-H-3'), $4.26(1 \mathrm{H}, \mathrm{dd}, J=3.6,12.4 \mathrm{~Hz}$, AZT-H-5a'), $4.44(1 \mathrm{H}, \mathrm{dd}, J=4.4,12.4$ Hz, AZT-H-5b'), 4.47 (1H, dd, $J=4.8,10.8 \mathrm{~Hz}, \mathrm{H}-3), 6.15$ (1H, t-like, $J=6.4 \mathrm{~Hz}$, AZT$\left.\mathrm{H}-1^{\prime}\right), 6.89\left(1 \mathrm{H}, \mathrm{s}, \mathrm{CH}(\mathrm{Ph})_{2}\right), 7.22-7.34(11 \mathrm{H}, \mathrm{m}$, aromatic-H and AZT-H-6), $8.97(1 \mathrm{H}, \mathrm{br}$ s, AZT-NH); ${ }^{13} \mathrm{C}$ NMR $\left(\mathrm{CDCl}_{3}, 100 \mathrm{MHz}\right) \delta 12.6\left(\mathrm{AZT}^{\left.-\mathrm{CH}_{3}\right)}, 14.6(\mathrm{C}-27), 14.9(\mathrm{C}-29), 16.0\right.$ (C-26, -25), 16.6 (C-24), 18.1 (C-6), 20.7 (C-11), 21.6 (C-21), 22.9 (C-30), 23.7 (C-2), 26.8 (C-12), 26.9 (C-15), 27.6 (3-O-dimethylglutaryl $\mathrm{CH}_{3}$ ), 27.9, 28.0 (28-O-dimethylglutaryl $\mathrm{CH}_{3}$ ), 28.0 (C-23), 29.4 (C-20), 29.9 (C-16), 32.6 (28-O-dimethylglutaryl C-3"), 32.8 (3-Odimethylglutaryl C-3'), 34.2 (C-7), 34.7 (C-22), 37.0 (C-10), 37.1 (C-13), 37.5 (AZT-C-2'), 37.6 (C-4), 38.3 (C-1), 40.9 (C-8), 42.8 (C-14), 44.5 (28-O-dimethylglutaryl C-4" and $\mathrm{C}-19), 45.0$ (28-O-dimethylglutaryl C-2"), 45.5 (3-O-glutaryl C-4'), 45.8 (3-O-glutaryl C-2'), 46.4 (C-17), 48.1 (C-18), 49.9 (C-9), 55.3 (C-5), 60.4 (AZT-C-3'), 62.7 (C-28, and AZTC-5'), 76.7 ( $\left.\mathrm{CH}(\mathrm{Ph})_{2}\right), 80.9$ (C-3), 81.8 (AZT-C-4'), 85.2 (AZT-C-1'), 111.3 (AZT-C-5), 127.1 (benzhydryl C-2', 6'), 127.8 (benzyhydryl C-4'), 128.4 (benzhydryl C-3', 5'), 135.3 (AZT-C-6), 140.2 (benzhydryl C-1'), 150.0 (AZT-C-2), 163.6 (AZT-C-4), 170.8 (3-Odimethylglutaryl 5'-COO-), 171.2 (28-O-dimethylglutaryl 5"-COO-AZT), 171.6 (3-Odimethylglutaryl 1'-COO-), 172.3 (28-O-dimethylglutaryl 1"-COO-). HRESIMS (positive) $\mathrm{m} / z 1144.6917[\mathrm{M}+\mathrm{H}]^{+}$(calcd for $\mathrm{C}_{67} \mathrm{H}_{94} \mathrm{~N}_{5} \mathrm{O}_{11}, 1144.6950$ ).

4.2.9 General procedure for removing benzhydryl group (49-62)-The final products were prepared by refluxing of a solution of benzdryl ester derivative in $80 \%$ HOAc 
(2-6 mL) overnight. After cooling to room temperature, the reaction mixture was extracted with $\mathrm{CHCl}_{3}$, and the organic layer was washed with water and brine, dried over $\mathrm{Na}_{2} \mathrm{SO}_{4}$ and concentrated. The residue was purified by preparative HPLC (cholester, $\mathrm{MeOH} / \mathrm{H}_{2} \mathrm{O} / \mathrm{HOAc}$ $=93: 6: 1)$.

\section{3'-Azido-3'-deoxythymidine-5'-yl 3-O-(3',3'-dimethylsuccinyl)-lup-20(29)en-28-yl}

succinate (49): Yield $46.5 \%$ (starting from $62 \mathrm{mg}$ of 35); white solid. $[\alpha]_{\cap}^{18}+7.2^{\circ}(c 0.57$, $\left.\mathrm{CHCl}_{3}\right) .{ }^{1} \mathrm{H}$ NMR $\left(\mathrm{CDCl}_{3}, 500 \mathrm{MHz}\right) \delta 0.80\left(3 \mathrm{H}, \mathrm{s}, \mathrm{CH}_{3}-24\right), 0.82\left(3 \mathrm{H}, \mathrm{s}, \mathrm{CH}_{3}-23\right), 0.83$ (3H, s, $\left.\mathrm{CH}_{3}-25\right), 0.96$ (3H, s, $\left.\mathrm{CH}_{3}-27\right), 1.00$ (3H, s, $\left.\mathrm{CH}_{3}-26\right), 1.29,1.31$ (each 3H, s, 3-Odimethylsuccinyl $\left.\mathrm{CH}_{3}\right), 1.68\left(3 \mathrm{H}, \mathrm{s}, \mathrm{CH}_{3}-30\right), 1.94\left(3 \mathrm{H}, \mathrm{d}, J=0.9 \mathrm{~Hz}, \mathrm{AZT}-\mathrm{CH}_{3}\right), 2.33-$ $2.49\left(3 \mathrm{H}, \mathrm{m}, \mathrm{AZT}-\mathrm{H}_{2}-2^{\prime}\right.$ and $\left.\mathrm{H}-19\right), 2.57,2.68$ (each $1 \mathrm{H}, \mathrm{d}, J=15.6 \mathrm{~Hz}, 3-O-$ dimethylsuccinyl $\left.\mathrm{H}_{2}-2^{\prime}\right), 2.67-2.77$ (4H, m, 28-O-succinyl $\mathrm{H}_{2}-2$ ", 3"), 3.86, 4.30 (each $1 \mathrm{H}$, d, $\left.J=11.2 \mathrm{~Hz}, \mathrm{H}_{2}-28\right), 4.05$ (1H, q-like, AZT-H-4'), 4.21-4.25 (1H, m, AZT-H-3'), 4.30 $\left(1 \mathrm{H}, \mathrm{dd}, J=3.4,12.3 \mathrm{~Hz}\right.$, AZT-H $\left.-5 \mathrm{a}^{\prime}\right), 4.49(1 \mathrm{H}, \mathrm{dd}, J=5.2,11.2 \mathrm{~Hz}, \mathrm{H}-3), 4.52(1 \mathrm{H}, \mathrm{dd}, J$ $\left.=4.3,12.3 \mathrm{~Hz}, \mathrm{AZT}-\mathrm{H}_{2}-5 \mathrm{~b}^{\prime}\right), 4.58,4.68$ (each $\left.1 \mathrm{H}, \mathrm{br} \mathrm{s}, \mathrm{H}_{2}-29\right), 6.14(1 \mathrm{H}, \mathrm{t}-\mathrm{like}, J=6.3 \mathrm{~Hz}$, AZT-H-1'), $7.30(1 \mathrm{H}, \mathrm{d}, J=1.2 \mathrm{~Hz}$, AZT-H- 6$), 8.68\left(1 \mathrm{H}, \mathrm{br} \mathrm{s}\right.$, AZT-NH); ${ }^{13} \mathrm{C}$ NMR $\left(\mathrm{CDCl}_{3}, 125 \mathrm{MHz}\right) \delta 12.6\left(\mathrm{AZT}-\mathrm{CH}_{3}\right), 14.7(\mathrm{C}-27), 16.0(\mathrm{C}-26), 16.1$ (C-25), $16.5(\mathrm{C}-24)$, 18.1 (C-6), 19.1 (C-30), 20.8 (C-11), 23.6 (C-2), 25.1 (C-12), 25.1, 25.6 (3-Odimethylsuccinyl $\mathrm{CH}_{3}$ ), 27.0 (C-15), 27.9 (C-23), 29.0 (28-O-succinyl C-2", 3"), 29.5 (C-21), 29.7 (C-16), 34.1 (C-7), 34.6 (C-22), 37.0 (C-10), 37.6 (AZT-C-2' and C-13), 37.7 (C-4), 38.3 (C-1), 40.4 (dimethylsuccinyl C-3'), 409 (C-8), 42.7 (C-14), 44.7 (3-Odimethylsuccinyl C-2'), 46.4 (C-17), 47.7 (C-19), 48.7 (C-18), 50.2 (C-9), 55.4 (C-5), 60.1 (AZT-C-3'), 63.1 (AZT-C-5'), 63.4 (C-28), 81.5 (C-3), 81.9 (AZT-C-4'), 85.2 (AZT-C-1'), 110.0 (C-29), 111.3 (AZT-C-5), 135.4 (AZT-C-6), 149.9 (AZT-C-2), 150.0 (C-20), 163.5 (AZT-C-4), 171.1 (3-O-dimethylsuccinyl 1'-COO-), 172.0 (28- $O$-succinyl 4"-COO-AZT), 172.6 (28- $O$-succinyl 1"-COO-), 180.8 (3-O-dimethylsuccinyl 4'-COOH). HRESIMS (positive) $\mathrm{m} / z$. $942.5218[\mathrm{M}+\mathrm{Na}]^{+}$(calcd for $\mathrm{C}_{50} \mathrm{H}_{73} \mathrm{~N}_{5} \mathrm{O}_{11} \mathrm{Na}, 942.5204$ ).

1-(3'-Azido-3'-deoxythymidine-5'-yl)-4-[3-O-(3',3'-dimethylsuccinyl)-lup-20(29)en-28yl] 2,2-dimethylsuccinate (50) and 4-(3'-Azido-3'-deoxythymidine-5'-yl)-1-[3-O-(3',3'dimethylsuccinyl)-lup-20(29)en-28-yl] 2,2-dimethylsuccinate (51): Total yield 78.9\% (starting from $105 \mathrm{mg}$ of mixture of $\mathbf{3 6}$ and $\mathbf{3 7}$ ); in a ratio of 2:1; white solid.

Compound 50: $[\alpha]_{\mathrm{n}}^{29}+8.2^{\circ}\left(c 2.38 \mathrm{CHCl}_{3}\right) .{ }^{1} \mathrm{H} \mathrm{NMR}\left(\mathrm{CDCl}_{3}, 400 \mathrm{MHz}\right) \delta 0.81(3 \mathrm{H}, \mathrm{s}$, $\mathrm{CH}_{3}-24$ ), 0.83 (each 3H, s, $\left.\mathrm{CH}_{3}-23,25\right), 0.96$ ( $3 \mathrm{H}, \mathrm{s}, \mathrm{CH}_{3}-27$ ), 1.00 (3H, s, $\mathrm{CH}_{3}-26$ ), 1.29, 1.30 (each $3 \mathrm{H}, \mathrm{s}, 3-O$-dimethylsuccinyl $\mathrm{CH}_{3}$ ), 1.30, 1.31 (each $3 \mathrm{H}, \mathrm{s}, 28-O$ -

dimethylsuccinyl $\left.\mathrm{CH}_{3}\right), 1.68\left(3 \mathrm{H}, \mathrm{s}, \mathrm{CH}_{3}-30\right), 1.93\left(3 \mathrm{H}\right.$, br s, AZT- $\left.\mathrm{CH}_{3}\right), 2.26-2.33,2.44-$ 2.50 (each $\left.1 \mathrm{H}, \mathrm{m}, \mathrm{AZT}-\mathrm{H}_{2}-2^{\prime}\right), 2.37-2.44(1 \mathrm{H}, \mathrm{m}, \mathrm{H}-19), 2.56,2.68$ (each 1H, d, $J=16.0$ $\mathrm{Hz}, 3$ - $O$-dimethylsuccinyl $\mathrm{H}_{2}-2^{\prime}$ ), 2.60, 2.74 (each $1 \mathrm{H}, \mathrm{d}, J=16.4 \mathrm{~Hz}, 28-O$ dimethylsuccinyl $\mathrm{H}_{2}-2$ "), 3.84, 4.27 (each $1 \mathrm{H}, \mathrm{d}, J=11.2 \mathrm{~Hz}, \mathrm{H}_{2}-28$ ), 4.09 (1H, q-like, AZT-H-4'), 4.25-4.31 (2H, m, AZT-H-3' and AZT-H-5a'), $4.51(1 \mathrm{H}, \mathrm{dd}, J=4.0,12.0 \mathrm{~Hz}$, AZT-H-5b'), $4.48\left(1 \mathrm{H}, \mathrm{dd}, J=5.6,11.2 \mathrm{~Hz}, \mathrm{H}-3\right.$ ), 4.59, 4.68 (each 1H, br s, $\left.\mathrm{H}_{2}-29\right), 6.16$ $(1 \mathrm{H}, \mathrm{t}-\mathrm{like}, J=6.4 \mathrm{~Hz}$, AZT-H-1'), $7.28(1 \mathrm{H}, \mathrm{d}, J=0.8 \mathrm{~Hz}$, AZT-H-6), $9.38(1 \mathrm{H}, \mathrm{br}$, AZT$\mathrm{NH}) ;{ }^{13} \mathrm{C} \mathrm{NMR}\left(\mathrm{CDCl}_{3}, 100 \mathrm{MHz}\right) \delta 12.4$ (AZT-CH3), 14.7 (C-27), 15.9 (C-26), 16.1 (C-25), 16.5 (C-24), 18.1 (C-6), 19.1 (C-30), 20.7 (C-11), 23.6 (C-2), 25.1 (C-12), 25.1, 25.6 (3-O-dimethylsuccinyl $\left.\mathrm{CH}_{3}\right), 25.1,25.9$ (28-O-dimethylsuccinyl $\left.\mathrm{CH}_{3}\right), 27.0(\mathrm{C}-15), 27.9$ (C-23), 29.5 (C-21), 29.7 (C-16), 34.1 (C-7), 34.5 (C-22), 37.0 (C-10), 37.5 (AZT-C-2'), 37.6 (C-13), 37.7 (C-4), 38.4 (C-1), 40.5 (3-O-dimethylsuccinyl C-3'), 40.7 (28-Odimethylsuccinyl C-3"), 40.8 (C-8), 42.7 (C-14), 44.6 (28-O-dimethylsuccinyl C-2"), 44.7 (3-O-dimethylsuccinyl C-2'), 46.3 (C-17), 47.7 (C-19), 48.7 (C-18), 50.2 (C-9), 55.4 (C-5), 60.4 (AZT-C-3'), 63.1 (C-28), 63.0 (AZT-C-5'), 81.5 (C-3), 82.0 (AZT-C-4'), 84.9 (AZTC-1'), 110.0 (C-29), 111.3 (AZT-C-5), 135.2 (AZT-C-6), 149.9 (C-20), 150.1 (AZT-C-2), 
164.0 (AZT-C-4), 171.1 (3-O-dimethylsuccinyl 1'-COO-), 171.7 (28-O-dimethylsuccinyl 1"-COO-), 176.5 (28-O-dimethylsuccinyl 4"-COO-AZT), 182.2 (3-O-dimethylsuccinyl 4'$\mathrm{COOH}$ ). HRESIMS (positive) $\mathrm{m} / z$ $970.5511[\mathrm{M}+\mathrm{Na}]^{+}$(calcd for $\mathrm{C}_{52} \mathrm{H}_{77} \mathrm{~N}_{5} \mathrm{O}_{11} \mathrm{Na}$, 970.5517).

Compound 51: $[\alpha]_{\mathrm{D}}^{27}+15.6^{\circ}(c 4.68 \mathrm{CHCl} 3) .{ }^{1} \mathrm{H} \mathrm{NMR}(\mathrm{CDCl} 3,400 \mathrm{MHz}) \delta 0.80(3 \mathrm{H}, \mathrm{s}$, $\left.\mathrm{CH}_{3}-24\right), 0.82$ (6H, s, CH3-23, C-25), 0.96 (3H, s, $\left.\mathrm{CH}_{3}-27\right), 1.01$ (3H, s, $\left.\mathrm{CH}_{3}-26\right), 1.28$, 1.29 (each $3 \mathrm{H}, \mathrm{s}, 3-O$-dimethylsuccinyl $\mathrm{CH}_{3}$ ), 1.30, 1.33 (each $3 \mathrm{H}, \mathrm{s}, 28-O$ dimethylsuccinyl $\left.\mathrm{CH}_{3}\right), 1.67$ (3H, s, CH3-30), 1.94 (3H, br s, AZT-CH3), 2.33-2.50 (3H, $\mathrm{m}$, AZT- $\mathrm{H}_{2}-2$ and $\mathrm{H}-19$ ), 2.61, 2.65 (each $1 \mathrm{H}, \mathrm{d}, J=16.4 \mathrm{~Hz}, 28$ - $O$-dimethylsuccinyl $\mathrm{H}_{2}-3$ "), 2.56, 2.67 (each $1 \mathrm{H}, \mathrm{d}, J=15.6 \mathrm{~Hz}, 3-O$-dimethylsuccinyl $\mathrm{H}_{2}-2^{\prime}$ ), 3.83, 4.27 (each $\left.1 \mathrm{H}, \mathrm{d}, J=10.8 \mathrm{~Hz}, \mathrm{H}_{2}-28\right), 4.03$ (1H, q-like, AZT-H-4'), 4.18-4.22(1H, m, AZT-H-3'), 4.25 $(1 \mathrm{H}, \mathrm{dd}, J=4.0,12.4 \mathrm{~Hz}$, AZT-H-5a'), 4.41 (1H, dd, $J=4.4,12.4 \mathrm{~Hz}$, AZT-H-5b'), 4.48 $(1 \mathrm{H}, \mathrm{dd}, J=5.2,10.8 \mathrm{~Hz}, \mathrm{H}-3), 4.58,4.67$ (each $\left.1 \mathrm{H}, \mathrm{br} \mathrm{s}, \mathrm{H}_{2}-29\right), 6.12(1 \mathrm{H}, \mathrm{t}$-like, $J=6.4$ Hz, AZT-H-1'), $7.31\left(1 \mathrm{H}, \mathrm{d}, J=0.8 \mathrm{~Hz}\right.$, AZT-H-6), $9.95\left(1 \mathrm{H}, \mathrm{br}\right.$ s, AZT-NH); ${ }^{13} \mathrm{C}$ NMR $\left(\mathrm{CDCl}_{3}, 100 \mathrm{MHz}\right) \delta 12.5\left(\mathrm{AZT}-\mathrm{CH}_{3}\right), 14.7$ (C-27), 15.9 (C-26), 16.0 (C-25), 16.4 (C-24), 18.1 (C-6), 19.1 (C-30), 20.7 (C-11), 23.5 (C-2), 25.1 (C-12), 24.9, 25.6 (3-Odimethylsuccinyl $\left.\mathrm{CH}_{3}\right), 25.4,25.7$ (28-O-dimethylsuccinyl $\left.\mathrm{CH}_{3}\right), 26.9(\mathrm{C}-15), 27.8(\mathrm{C}-23)$, 29.5 (C-21), 29.8 (C-16), 34.0 (C-7), 34.5 (C-22), 37.0 (C-10), 37.5 (AZT-C-2' and C-13), 37.6 (C-4), 38.3 (C-1), 40.4 (3-O-dimethylsuccinyl C-3'), 40.8 (C-8), 41.0 (28-Odimethylsuccinyl C-2"), 42.6 (C-14), 43.8 (28-O-dimethylsuccinyl C-3"), 44.7 (3-Odimethylsuccinyl C-2'), 46.5 (C-17), 47.7 (C-19), 48.8 (C-18), 50.2 (C-9), 55.3 (C-5), 60.2 (AZT-C-3'), 62.9 (AZT-C-5'), 63.2 (C-28), 81.5 (C-3), 81.8 (AZT-C-4'), 85.3 (AZT-C-1'), 109.9 (C-29), 111.2 (AZT-C-5), 135.6 (AZT-C-6), 149.9 (C-20), 150.2 (AZT-C-2), 164.4 (AZT-C-4), 170.7 (28-O-dimethylsuccinyl 4"-COO-AZT), 171.0 (3-O-dimethylsuccinyl 1'COO-), 176.9 (28-O-dimethylsuccinyl 1"-COO-), 182.7 (3-O-dimethylsuccinyl 4'-COOH). HRESIMS (positive) $\mathrm{m} / z, 970.5490[\mathrm{M}+\mathrm{Na}]^{+}$(calcd for $\mathrm{C}_{52} \mathrm{H}_{77} \mathrm{~N}_{5} \mathrm{O}_{11} \mathrm{Na}, 970.5517$ ).

\section{3'-Azido-3'-deoxythymidine-5'-yl 3-O-(3',3'-dimethylsuccinyl)-lup-20(29)en-28-yl}

glutarate (52): Yield $42.7 \%$ (starting from $90 \mathrm{mg}$ of 38); white solid. $[\alpha]_{\mathrm{D}}^{15}+18.8^{\circ}(c 0.6$, $\left.\mathrm{CHCl}_{3}\right) .{ }^{1} \mathrm{H} \mathrm{NMR}\left(\mathrm{CDCl}_{3}, 500 \mathrm{MHz}\right) \delta 0.81\left(3 \mathrm{H}, \mathrm{s}, \mathrm{CH}_{3}-24\right), 0.83\left(3 \mathrm{H}, \mathrm{s}, \mathrm{CH}_{3}-23\right), 0.83$ $\left(3 \mathrm{H}, \mathrm{s}, \mathrm{CH}_{3}-25\right), 0.96$ (3H, s, $\left.\mathrm{CH}_{3}-27\right), 1.02\left(3 \mathrm{H}, \mathrm{s}, \mathrm{CH}_{3}-26\right), 1.29,1.31$ (each $3 \mathrm{H}, \mathrm{s}, 3-\mathrm{O}-$ dimethylsuccinyl $\left.\mathrm{CH}_{3}\right), 1.68\left(3 \mathrm{H}, \mathrm{s}, \mathrm{CH}_{3}-30\right), 1.94\left(3 \mathrm{H}, \mathrm{d}, J=1.2 \mathrm{~Hz}, \mathrm{AZT}-\mathrm{CH}_{3}\right), 1.99(2 \mathrm{H}$, quint, $J=7.2 \mathrm{~Hz}, 28-O$-glutaryl $\left.\mathrm{H}_{2}-3 "\right), 2.33-2.49\left(3 \mathrm{H}, \mathrm{m}, \mathrm{AZT}-\mathrm{H}_{2}-2^{\prime}\right.$ and $\left.\mathrm{H}-19\right), 2.42(2 \mathrm{H}$, t, $J=7.2 \mathrm{~Hz}, 28$ - $O$-glutaryl $\left.\mathrm{H}_{2}-2 "\right), 2.46\left(2 \mathrm{H}, \mathrm{t}, J=7.2 \mathrm{~Hz}, 28\right.$ - $O$-glutaryl $\left.\mathrm{H}_{2}-4 "\right), 2.57,2.67$ (each $1 \mathrm{H}, \mathrm{d}, J=15.8 \mathrm{~Hz}, 3-O$-dimethylsuccinyl $\mathrm{H}_{2}-2^{\prime}$ ), 3.85, 4.28 (each $1 \mathrm{H}, \mathrm{d}, J=10.8 \mathrm{~Hz}$, $\left.\mathrm{H}_{2}-28\right), 4.07$ (1H, q-like, AZT-H-4'), 4.20-4.24 (1H, m, AZT-H-3'), $4.32(1 \mathrm{H}, \mathrm{dd}, J=3.9$, $\left.12.3 \mathrm{~Hz}, \mathrm{AZT}-\mathrm{H}_{2}-5 \mathrm{a}^{\prime}\right), 4.40\left(1 \mathrm{H}, \mathrm{dd}, J=4.7,12.3 \mathrm{~Hz}, \mathrm{AZT}-\mathrm{H}_{2}-5 \mathrm{~b}^{\prime}\right), 4.49(1 \mathrm{H}, \mathrm{dd}, J=5.2$, $11.2 \mathrm{~Hz}, \mathrm{H}-3$ ), 4.59, 4.68 (each 1H, br s, $\mathrm{H}_{2}-29$ ), 6.09 (1H, t-like, $J=6.3 \mathrm{~Hz}$, AZT-H-1'), $7.21\left(1 \mathrm{H}, \mathrm{d}, J=1.1 \mathrm{~Hz}\right.$, AZT-H-6); ${ }^{13} \mathrm{C}$ NMR $\left(\mathrm{CDCl}_{3}, 125 \mathrm{MHz}\right) \delta 12.6\left(\mathrm{AZT}^{-\mathrm{CH}_{3}}\right), 14.7$ (C-27), 16.0 (C-26), 16.1 (C-25), 16.5 (C-24), 18.1 (C-6), 19.1 (C-30), 20.0 (28-O-glutaryl C-3"), 20.8 (C-11), 23.6 (C-2), 25.1 (C-12), 25.1, 25.6 (3-O-dimethylsuccinyl $\mathrm{CH}_{3}$ ), 27.0 (C-15), 27.9 (C-23), 29.5 (C-21), 29.8 (C-16), 33.1 (28-O-glutaryl C-4"), 33.2 (28-Oglutaryl C-2"), 34.1 (C-7), 34.6 (C-22), 37.0 (C-10), 37.5 (AZT-C-2'), 37.6 (C-13), 37.7 (C-4), 38.4 (C-1), 40.4 (dimethylsuccinyl C-3'), 40.9 (C-8), 42.7 (C-14), 44.8 (3- $O$ dimethylsuccinyl C-2'), 46.4 (C-17), 47.7 (C-19), 48.7 (C-18), 50.2 (C-9), 55.4 (C-5), 60.6 (AZT-C-3'), 63.0 (C-28), 63.3 (AZT-C-5'), 81.6 (C-3), 81.7 (AZT-C-4'), 85.7 (AZT-C-1'), 110.0 (C-29), 111.3 (AZT-C-5), 135.5 (AZT-C-6), 149.8 (C-20), 149.9 (AZT-C-2), 163.5 (AZT-C-4), 171.2 (3-O-dimethylsuccinyl 1'-COO-), 172.3 (28-O-glutaryl 5"-COO-AZT), 173.1 (28-O-glutaryl 1"-COO-), 180.8 (3-O-dimethylsuccinyl 4'-COOH). HRESIMS (positive) $\mathrm{m} / z$ 956.5361 $[\mathrm{M}+\mathrm{Na}]^{+}$(calcd for $\mathrm{C}_{51} \mathrm{H}_{75} \mathrm{~N}_{5} \mathrm{O}_{11} \mathrm{Na}, 956.5361$ ). 
3'-Azido-3'-deoxythymidine-5'-yl 3-O-(3',3'-dimethylsuccinyl)-lup-20(29)en-28-yl 3,3-

dimethylglutarate (53): Yield $87.3 \%$ (starting from $114 \mathrm{mg}$ of 39); white solid. $[\alpha]_{\mathrm{D}}^{27}$ $+15.3^{\circ}\left(c 2.84, \mathrm{CHCl}_{3}\right) .{ }^{1} \mathrm{H}$ NMR $\left(\mathrm{CDCl}_{3}, 400 \mathrm{MHz}\right) \delta 0.80\left(3 \mathrm{H}, \mathrm{s}, \mathrm{CH}_{3}-24\right), 0.83$ (each $3 \mathrm{H}$, s, $\left.\mathrm{CH}_{3}-23,25\right), 0.96\left(3 \mathrm{H}, \mathrm{s}, \mathrm{CH}_{3}-27\right), 1.01\left(3 \mathrm{H}, \mathrm{s}, \mathrm{CH}_{3}-26\right), 1.12,1.15$ (each $3 \mathrm{H}, \mathrm{s}, 28-O-$ dimethylglutaryl $\mathrm{CH}_{3}$ ), 1.29, 1.30 (each $3 \mathrm{H}, \mathrm{s}, 3$-O-dimethylsuccinyl $\left.\mathrm{CH}_{3}\right), 1.68(3 \mathrm{H}, \mathrm{s}$, $\left.\mathrm{CH}_{3}-30\right), 1.94$ (3H, br s, AZT-CH3), 2.30-2.35, 2.40-2.51 (2H, m, AZT-H ${ }_{2}-2$ '), 2.35-2.42 (1H, m, H-19), 2.44, 2.49 (each $1 \mathrm{H}, \mathrm{d}, J=16.0 \mathrm{~Hz}, 28$ - $O$-dimethylglutaryl $\mathrm{H}_{2}-2$ "), 2.49, 2.55 (each $1 \mathrm{H}, \mathrm{d}, J=16.0 \mathrm{~Hz}, 28$ - $O$-dimethylglutaryl $\mathrm{H}_{2}-4 "$ ), 2.56, 2.68 (each $1 \mathrm{H}, \mathrm{d}, J=$ $16.0 \mathrm{~Hz}, 3$ - $O$-dimethylsuccinyl $\mathrm{H}_{2}-2^{\prime}$ ), 3.82, 4.26 (each $\left.1 \mathrm{H}, \mathrm{d}, J=11.2 \mathrm{~Hz}, \mathrm{H}_{2}-28\right), 4.07$ (1H, q-like, AZT-H-4'), 4.20-4.25 (1H, m, AZT-H-3'), 4.26 (1H, dd, $J=4.0,12.0 \mathrm{~Hz}$, AZTH-5a'), $4.43(1 \mathrm{H}, \mathrm{dd}, J=4.4,12.0 \mathrm{~Hz}$, AZT-H-5b'), $4.48(1 \mathrm{H}, \mathrm{dd}, J=5.2,10.8 \mathrm{~Hz}, \mathrm{H}-3)$, 4.60, 4.69 (each 1H, br s, $\left.\mathrm{H}_{2}-29\right), 6.14(1 \mathrm{H}, \mathrm{t}-\mathrm{like}, J=6.4 \mathrm{~Hz}$, AZT-H-1'), $7.29(1 \mathrm{H}, \mathrm{d}, J=$ $1.2 \mathrm{~Hz}$, AZT-H-6), $9.43\left(1 \mathrm{H}\right.$, br s, AZT-NH); ${ }^{13} \mathrm{C} \mathrm{NMR}\left(\mathrm{CDCl}_{3}, 100 \mathrm{MHz}\right) \delta 12.5$ (AZT$\mathrm{CH}_{3}$ ), 14.7 (C-27), 16.0 (C-26), 16.1 (C-25), 16.5 (C-24), 18.1 (C-6), 19.1 (C-30), 20.8 (C-11), 23.6 (C-2), 25.1 (C-12), 25.0, 25.6 (3-O-dimethylsuccinyl $\left.\mathrm{CH}_{3}\right), 27.0(\mathrm{C}-15), 27.9$ (28-O-dimethylglutaryl $\left.\mathrm{CH}_{3}\right), 28.0(\mathrm{C}-23), 29.5(\mathrm{C}-21), 29.8(\mathrm{C}-16), 32.6(28-O$ dimethylglutaryl C-3"), 34.1 (C-7), 34.6 (C-22), 37.0 (C-10), 37.5 (AZT-C-2', C-13), 37.7 (C-4), 38.4 (C-1), 40.4 (3-O-dimethylsuccinyl C-3'), 40.8 (C-8), 42.7 (C-14), 44.5 (28-Odimethylglutaryl C-4"), 44.7 (3-O-dimethylsuccinyl C-2'), 45.0 (28-O-dimethylglutaryl C-2"), 46.2 (C-17), 47.7 (C-19), 48.7 (C-18), 50.2 (C-9), 55.4 (C-5), 60.4 (AZT-C-3'), 62.7 (C-28), 62.8 (AZT-C-5'), 81.5 (C-3), 81.8 (AZT-C-4'), 85.3 (AZT-C-1'), 109.9 (C-29), 111.2 (AZT-C-5), 135.4 (AZT-C-6), 150.0 (C-20), 150.1 (AZT-C-2), 163.9 (AZT-C-4), 171.0 (3$O$-dimethylsuccinyl 1'-COO-), 171.2 (28-O-dimethylglutaryl 5"-COO-AZT), 172.3 (28- $O$ dimethylglutaryl 1"-COO-), 182.1 (3-O-dimethylsuccinyl 4'-COOH). HRESIMS (positive) $m / z 962.5881[\mathrm{M}+\mathrm{H}]^{+}$(calcd for $\left.\mathrm{C}_{5} 3 \mathrm{H}_{80} \mathrm{~N}_{5} \mathrm{O}_{11}, 962.5854\right)$.

3'-Azido-3'-deoxythymidine-5'-yl 3-O-(3',3'-dimethylglutaryl)-lup-20(29)en-28-yl 3,3-

dimethylglutarate (54): Yield $84.2 \%$ (starting from $107 \mathrm{mg}$ of $\mathbf{4 0}$ ); white solid. $[\alpha]_{\mathrm{D}}^{26}$ $+15.6^{\circ}$ (c $\left.3.09 \mathrm{CHCl}_{3}\right) .{ }^{1} \mathrm{H} \mathrm{NMR}\left(\mathrm{CDCl}_{3}, 400 \mathrm{MHz}\right) \delta 0.84$ (each $\left.3 \mathrm{H}, \mathrm{s}, \mathrm{CH}_{3}-24,25\right), 0.85$ (6H, s, $\left.\mathrm{CH}_{3}-23\right), 0.97$ (3H, s, $\left.\mathrm{CH}_{3}-27\right), 1.02$ ( $3 \mathrm{H}, \mathrm{s}, \mathrm{CH}_{3}-26$ ), 1.12, 1.15 (each $3 \mathrm{H}, \mathrm{s}, 28-O-$ dimethylglutaryl $\left.\mathrm{CH}_{3}\right), 1.15\left(6 \mathrm{H}, \mathrm{s}, 3-O\right.$-dimethylglutaryl $\left.\mathrm{CH}_{3}\right), 1.68\left(3 \mathrm{H}, \mathrm{s}, \mathrm{CH}_{3}-30\right), 1.94$ (3H, br s, AZT-CH3), 2.32-2.51 (3H, m, AZT-H ${ }_{2}-2$ and H-19), 2.40, 2.47 (each 1H, d, $J=$ $14.4 \mathrm{~Hz}, 3$ - $O$-dimethylglutaryl $\left.\mathrm{H}_{2}-2^{\prime}\right), 2.46\left(2 \mathrm{H}, \mathrm{s}, 3-O\right.$-dimethylglutaryl $\mathrm{H}_{2}-4$ '), $2.44,2.49$ (each $1 \mathrm{H}, \mathrm{d}, J=14.4 \mathrm{~Hz}, 28-O$-dimethylglutaryl $\left.\mathrm{H}_{2}-2 "\right), 2.48,2.56$ (each $1 \mathrm{H}, \mathrm{d}, J=14.4 \mathrm{~Hz}$, 28-O-dimethylglutaryl $\mathrm{H}_{2}-3$ "), 3.81, 4.26 (each $\left.1 \mathrm{H}, \mathrm{d}, J=11.2 \mathrm{~Hz}, \mathrm{H}_{2}-28\right), 4.07$ (1H, q-like, AZT-H-4'), 4.20-4.28 (1H, m, AZT-H-3'), 4.26 (1H, dd, $J=4.0,12.4 \mathrm{~Hz}$, AZT-H-5a'), 4.43 $(1 \mathrm{H}, \mathrm{dd}, J=4.0,12.4 \mathrm{~Hz}$, AZT-H-5b'), 4.49 (1H, dd, $J=5.2,11.6 \mathrm{~Hz}, \mathrm{H}-3), 4.60,4.69$ (each $1 \mathrm{H}$, br s, $\left.\mathrm{H}_{2}-29\right), 6.14$ (1H, t-like, $J=6.4 \mathrm{~Hz}$, AZT-H-1'), 7.29 (1H, br s, AZT-H-6), 9.41 $\left(1 \mathrm{H}\right.$, br s, AZT-NH); ${ }^{13} \mathrm{C}$ NMR $\left(\mathrm{CDCl}_{3}, 100 \mathrm{MHz}\right) \delta 12.5\left(\mathrm{AZT}-\mathrm{CH}_{3}\right), 14.7(\mathrm{C}-27), 16.0$ (C-26), 16.1 (C-25), 16.6 (C-24), 18.1 (C-6), 19.1 (C-30), 20.8 (C-11), 23.7 (C-2), 25.1 (C-12), 27.0 (C-15), 27.9 (3-O-dimethylglutaryl $\mathrm{CH}_{3}$ ), 27.9, 28.0 (28-O-dimethylglutaryl $\mathrm{CH}_{3}$ ), 28.0 (C-23), 29.5 (C-21), 29.8 (C-16), 32.6 (28-O-dimethylglutaryl C-3", 3-Odimethylglutaryl C-3'), 34.1 (C-7), 34.6 (C-22), 37.0 (C-10), 37.5 (AZT-C-2', C-13), 37.6 (C-4), 38.3 (C-1), 40.8 (C-8), 42.7 (C-14), 44.5 (28-O-dimethylglutaryl C-4"), 45.0 (28-Odimethylsuccinyl C-2"), 45.2 (3-O-dimethylglutaryl C-4'), 45.6 (3-O-dimethylglurayl C-2'), 46.2 (C-17), 47.7 (C-19), 48.7 (C-18), 50.2 (C-9), 55.4 (C-5), 60.4 (AZT-C-3'), 62.6 (C-28), 62.7 (AZT-C-5'), 81.3 (C-3), 81.8 (AZT-C-4'), 85.3 (AZT-C-1'), 109.9 (C-29), 111.2 (AZTC-5), 135.4 (AZT-C-6), 150.0 (C-20), 150.1 (AZT-C-2), 164.0 (AZT-C-4), 171.2 (28-Odimethylglutaryl 5"-COO-AZT), 172.3 (3-O-dimethylglutaryl 1'-COO-, 28-Odimethylglutaryl 1"-COO-), 176.0 (3-O-dimethylglutaryl 5'-COOH). HRESIMS (positive) $\mathrm{m} / \mathrm{z} 998.5824[\mathrm{M}+\mathrm{Na}]^{+}$(calcd for $\mathrm{C}_{54} \mathrm{H}_{81} \mathrm{~N}_{5} \mathrm{O}_{11} \mathrm{Na}, 998.5830$ ). 
1-(3'-Azido-3'-deoxythymidine-5'-yl)-4-[3-O-(3',3'-dimethylsuccinyl)-lup-28-yl] 2,2dimethylsuccinate (55) and 4-(3'-Azido-3'-deoxythymidine-5'-yl)-1-[3-O-(3',3'dimethylsuccinyl)-lup-28-yl] 2,2-dimethylsuccinate (56): Total yield 56.7\% (starting from $148 \mathrm{mg}$ of mixture of $\mathbf{4 1}$ and $\mathbf{4 2}$ ); in a ratio of 1.1:1; white solid.

Compound 55: $[\alpha]_{\mathrm{D}}^{30}-4.2^{\circ}\left(c 2.99 \mathrm{CHCl}_{3}\right) .{ }^{1} \mathrm{H} \mathrm{NMR}\left(\mathrm{CDCl}_{3}, 400 \mathrm{MHz}\right) \delta 0.76,0.83$ (each $3 \mathrm{H}, \mathrm{d}, J=8.0 \mathrm{~Hz}, \mathrm{CH} 3-29,30), 0.82\left(3 \mathrm{H}, \mathrm{s}, \mathrm{CH}_{3}-24\right), 0.84\left(6 \mathrm{H}, \mathrm{s}, \mathrm{CH}_{3}-23, \mathrm{CH}_{3}-25\right), 0.94$ $\left(3 \mathrm{H}, \mathrm{s}, \mathrm{CH}_{3}-27\right), 1.01\left(3 \mathrm{H}, \mathrm{s}, \mathrm{CH}_{3}-26\right), 1.31\left(6 \mathrm{H}, \mathrm{s}, 3-O\right.$-dimethylsuccinyl $\left.\mathrm{CH}_{3}\right), 1.30,1.31$ (each $1 \mathrm{H}, \mathrm{s}, 28-O$-dimethylsuccinyl $\mathrm{CH}_{3}$ ), 1.93 (3H, br s, AZT-CH ${ }_{3}$ ), 2.25-2.32, 2.44-2.50 (each $1 \mathrm{H}, \mathrm{m}, \mathrm{AZT}-\mathrm{H}_{2}-2^{\prime}$ ), 2.57, 2.68 (each $1 \mathrm{H}, \mathrm{d}, J=15.6 \mathrm{~Hz}, 3$ - $O$-dimethylsuccinyl $\mathrm{H}_{2}-2^{\prime}$ ), 2.59, 2.73 (each $1 \mathrm{H}, \mathrm{d}, J=16.4 \mathrm{~Hz}, 28-O$-dimethylsuccinyl $\mathrm{H}_{2}-2$ "), 3.81, 4.27 (each $1 \mathrm{H}, \mathrm{d}, J$ $\left.\left.=10.8 \mathrm{~Hz}, \mathrm{H}_{2}-28\right), 4.09\left(1 \mathrm{H}, \mathrm{q}-\mathrm{like}, \mathrm{AZT}-\mathrm{H}-4{ }^{\prime}\right), 4.25-4.29(1 \mathrm{H}, \mathrm{m}, \mathrm{AZT}-\mathrm{H}-3)^{\prime}\right), 4.28(1 \mathrm{H}$, dd, $J=4.4,12.0 \mathrm{~Hz}$, AZT-H-5a'), $4.52(1 \mathrm{H}, \mathrm{dd}, J=3.6,12.0 \mathrm{~Hz}$, AZT-H-5b'), 4.49 (1H, dd, $J=5.2,10.8 \mathrm{~Hz}, \mathrm{H}-3), 6.16(1 \mathrm{H}, \mathrm{t}-\mathrm{like}, J=6.4 \mathrm{~Hz}, \mathrm{AZT}-\mathrm{H}-1$ '), 7.27 (1H, br s, AZT-H-6), $9.31\left(1 \mathrm{H}\right.$, br s, AZT-NH); ${ }^{13} \mathrm{C} \mathrm{NMR}\left(\mathrm{CDCl}_{3}, 100 \mathrm{MHz}\right) \delta 12.4\left(\mathrm{AZT}^{-\mathrm{CH}_{3}}\right), 14.6(\mathrm{C}-27)$, 14.8 (C-29), 16.0 (C-26, C-25), 16.5 (C-24), 18.1 (C-6), 20.8 (C-11), 21.6 (C-21), 22.8 (C-30), 23.6 (C-2), 25.1, 25.6 (3-O-dimethylsuccinyl $\left.\mathrm{CH}_{3}\right), 25.0,25.8(28-O-$ dimethylsuccinyl $\mathrm{CH}_{3}$ ), 26.8 (C-12), 26.9 (C-15), 27.9 (C-23), 29.4 (C-20), 29.8 (C-16), 34.2 (C-7), 34.6 (C-22), 37.0 (C-10), 37.2 (C-13), 37.5 (AZT-C-2'), 37.7 (C-4), 38.4 (C-1), 40.4 (3- $O$-dimethylsuccinyl C-3'), 40.7 (28-O-dimethylsuccinyl C-3"), 40.9 (C-8), 42.8 (C-14), 44.5 (C-19), 44.6 (28-O-dimethylsuccinyl C-2"), 44.7 (3-O-dimethylsuccinyl C-2'), 46.5 (C-17), 48.1 (C-18), 49.9 (C-9), 55.4 (C-5), 60.4 (AZT-C-3'), 63.1 (C-28), 63.3 (AZTC-5'), 81.5 (C-3), 82.0 (AZT-C-4'), 84.9 (AZT-C-1'), 111.3 (AZT-C-5), 135.2 (AZT-C-6), 150.2 (AZT-C-2), 164.0 (AZT-C-4), 171.0 (3-O-dimethylsuccinyl 1'-COO-), 171.7 (28-Odimethylsuccinyl 1"-COO-), 176.5 (28-O-dimethylsuccinyl 4"-COO-AZT), 182.0 (3-Odimethylsuccinyl 4'-COOH). HRESIMS (positive) $\mathrm{m} / \mathrm{z} 950.5848[\mathrm{M}+\mathrm{H}]^{+}$(calcd for $\left.\mathrm{C}_{52} \mathrm{H}_{80} \mathrm{~N}_{5} \mathrm{O}_{11}, 950.5854\right)$.

Compound 56: $[\alpha]_{\mathrm{D}}^{27}-2.5^{\circ}\left(c 1.74 \mathrm{CHCl}_{3}\right) .{ }^{1} \mathrm{H} \mathrm{NMR}\left(\mathrm{CDCl}_{3}, 400 \mathrm{MHz}\right) \delta 0.77,0.84$ (each $3 \mathrm{H}, \mathrm{d}, J=8.0 \mathrm{~Hz}, \mathrm{CH} 3-29,30), 0.82\left(3 \mathrm{H}, \mathrm{s}, \mathrm{CH}_{3}-24\right), 0.85\left(6 \mathrm{H}, \mathrm{s}, \mathrm{CH}_{3}-23, \mathrm{CH}_{3}-25\right), 0.95$ (3H, s, $\left.\mathrm{CH}_{3}-27\right), 1.00\left(3 \mathrm{H}, \mathrm{s}, \mathrm{CH}_{3}-26\right), 1.31$ (each $3 \mathrm{H}, \mathrm{s}, 3$-O-dimethylsuccinyl $\mathrm{CH}_{3}$ ), 1.30, 1.33 (each $3 \mathrm{H}, \mathrm{s}, 28$-O-dimethylsuccinyl $\mathrm{CH}_{3}$ ), 1.95 (3H, br s, AZT-CH3), 2.32-2.39, 2.44 2.50 (each $1 \mathrm{H}, \mathrm{m}, \mathrm{AZT}-\mathrm{H}_{2}-2$ ) , 2.57, 2.68 (each $1 \mathrm{H}, \mathrm{d}, J=15.6 \mathrm{~Hz}, 3$ - $O$-dimethylsuccinyl $\mathrm{H}_{2}-2^{\prime}$ ), 2.61, 2.66 (each $1 \mathrm{H}, \mathrm{d}, J=16.0 \mathrm{~Hz}, 28-O$-dimethylsuccinyl $\mathrm{H}_{2}-3$ "), 3.81, 4.29 (each $\left.1 \mathrm{H}, \mathrm{d}, J=10.8 \mathrm{~Hz}, \mathrm{H}_{2}-28\right), 4.04$ (1H, q-like, AZT-H-4'), 4.17-4.22 (1H, m, AZT-H-3'), 4.26 $(1 \mathrm{H}, \mathrm{dd}, J=3.6,12.0 \mathrm{~Hz}$, AZT-H-5a'), $4.42(1 \mathrm{H}, \mathrm{dd}, J=4.4,12.0 \mathrm{~Hz}$, AZT-H-5b'), 4.50 $(1 \mathrm{H}, \mathrm{dd}, J=5.2,10.8 \mathrm{~Hz}, \mathrm{H}-3), 6.13\left(1 \mathrm{H}, \mathrm{t}-\mathrm{like}, J=6.4 \mathrm{~Hz}, \mathrm{AZT}-\mathrm{H}-1{ }^{\prime}\right), 7.30(1 \mathrm{H}, \mathrm{d}$, br s, AZT-H-6), $9.16\left(1 \mathrm{H}\right.$, br s, AZT-NH); ${ }^{13} \mathrm{C}$ NMR $\left(\mathrm{CDCl}_{3}, 100 \mathrm{MHz}\right) \delta 12.5($ AZT-CH 3$), 14.7$ (C-27), 14.9 (C-29), 16.0 (C-26, 25), 16.5 (C-24), 18.2 (C-6), 20.8 (C-11), 21.6 (C-21), 22.9 (C-30), 23.6 (C-2), 25.1, 25.6 (3-O-dimethylsuccinyl $\left.\mathrm{CH}_{3}\right), 25.5,25.8$ (28-Odimethylsuccinyl $\mathrm{CH}_{3}$ ), 26.8 (C-12), 26.9 (C-15), 27.9 (C-23), 29.4 (C-20), 30.0 (C-16), 34.2 (C-7), 34.7 (C-22), 37.0 (C-10), 37.2 (C-13), 37.5 (AZT-C-2'), 37.7 (C-4), 38.4 (C-1), 40.4 (3-O-dimethylsuccinyl C-3'), 40.9 (C-8), 41.1 (28-O-dimethylsuccinyl C-2"), 42.9 (C-14), 43.9 (28-O-dimethylsuccinyl C-3"), 44.6 (C-19), 44.7 (3-O-dimethylsuccinyl C-2'), 46.7 (C-17), 48.2 (C-18), 50.0 (C-9), 55.4 (C-5), 60.3 (AZT-C-3'), 63.0 (AZT-C-5'), 63.4 (C-28), 81.5 (C-3), 81.8 (AZT-C-4'), 85.3 (AZT-C-1'), 111.3 (AZT-C-5), 135.4 (AZT-C-6), 150.0 (AZT-C-2), 163.8 (AZT-C-4), 170.8 (28-O-dimethylsuccinyl 4"-COO-AZT), 171.0 (3-O-dimethylsuccinyl 1'-COO-), 176.9 (28-O-dimethylsuccinyl 1"-COO-), 181.7 (3-Odimethylsuccinyl 4'-COOH). HRESIMS (positive) $\mathrm{m} / \mathrm{z} 950.5873[\mathrm{M}+\mathrm{H}]^{+}$(calcd for $\left.\mathrm{C}_{52} \mathrm{H}_{80} \mathrm{~N}_{5} \mathrm{O}_{11}, 950.5854\right)$. 


\section{3'-Azido-3'-deoxythymidine-5'-yl 3-O-(3',3'-dimethylsuccinyl)-lup-28-yl 3,3-}

dimethylglutarate (57): Yield $45.6 \%$ (starting from $84 \mathrm{mg}$ of $\mathbf{4 3}$ ); white solid. $[\alpha]_{\mathrm{D}}^{27}-0.02^{\circ}$ (c 2.12, $\left.\mathrm{CHCl}_{3}\right) .{ }^{1} \mathrm{H} \mathrm{NMR}\left(\mathrm{CDCl}_{3}, 400 \mathrm{MHz}\right) \delta 0.77,0.84$ (each $3 \mathrm{H}, \mathrm{d}, J=8.0 \mathrm{~Hz}, \mathrm{CH}_{3}-29$, 30), 0.82 (3H, s, $\left.\mathrm{CH}_{3}-24\right), 0.85$ (6H, s, $\left.\mathrm{CH}_{3}-23, \mathrm{CH}_{3}-25\right), 0.95\left(3 \mathrm{H}, \mathrm{s}, \mathrm{CH}_{3}-27\right), 1.03(3 \mathrm{H}, \mathrm{s}$, $\mathrm{CH}_{3}$-26), 1.12, 1.15 (each $3 \mathrm{H}, \mathrm{s}, 28$ - $O$-dimethylglutaryl $\mathrm{CH}_{3}$ ), 1.30, 1.31 (each $3 \mathrm{H}, \mathrm{s}, 3-\mathrm{O}$ dimethylsuccinyl $\left.\mathrm{CH}_{3}\right), 1.95$ (3H, br s, AZT-CH3), 2.33-2.40, 2.43-2.50 (2H, m, AZT$\mathrm{H}_{2}-2^{\prime}$ ), 2.42, 2.48 (each $1 \mathrm{H}, \mathrm{d}, J=14.4 \mathrm{~Hz}, 28-O$-dimethylglutaryl $\left.\mathrm{H}_{2}-2 "\right), 2.49,2.56$ (each $1 \mathrm{H}, \mathrm{d}, J=15.2 \mathrm{~Hz}, 28$ - $O$-dimethylglutaryl $\mathrm{H}_{2}-4 "$ ), $2.57,2.68$ (each $1 \mathrm{H}, \mathrm{d}, J=15.6 \mathrm{~Hz}, 3-O-$ dimethylsuccinyl $\mathrm{H}_{2}-2$ '), 3.79, 4.26 (each $1 \mathrm{H}, \mathrm{d}, J=10.8 \mathrm{~Hz}, \mathrm{H}_{2}-28$ ), 4.07 (1H, q-like, AZTH-4'), 4.20-4.23 (1H, m, AZT-H-3'), 4.26 (1H, dd, $J=3.6,12.0 \mathrm{~Hz}$, AZT-H-5a'), $4.44(1 \mathrm{H}$, $\mathrm{dd}, J=4.4,12.0 \mathrm{~Hz}$, AZT-H-5b'), $4.50(1 \mathrm{H}, \mathrm{dd}, J=5.2,10.4 \mathrm{~Hz}, \mathrm{H}-3), 6.14$ (1H, t-like, $J=$ $6.4 \mathrm{~Hz}$, AZT-H-1'), 7.29 (1H, br s, AZT-H-6), $9.15\left(1 \mathrm{H}, \mathrm{br}\right.$ s, AZT-NH); ${ }^{13} \mathrm{C} \mathrm{NMR}\left(\mathrm{CDCl}_{3}\right.$, $100 \mathrm{MHz}) \delta 12.5\left(\mathrm{AZT}-\mathrm{CH}_{3}\right), 14.6(\mathrm{C}-27), 14.9(\mathrm{C}-29), 16.0(\mathrm{C}-26,25), 16.5(\mathrm{C}-24), 18.2$ (C-6), 20.8 (C-11), 21.6 (C-21), 22.9 (C-30), 23.6 (C-2), 25.1, 25.6 (3-O-dimethylsuccinyl $\left.\mathrm{CH}_{3}\right), 26.8$ (C-12), 26.9 (C-15), 27.9 (28-O-dimethylglutaryl $\left.\mathrm{CH}_{3}\right), 28.0(\mathrm{C}-23), 29.4$ (C-20), 29.9 (C-16), 32.6 (28-O-dimethylglutaryl C-3"), 34.2(C-7), 34.8 (C-22), 37.0 (C-10), 37.2 (C-13), 37.6 (AZT-C-2'), 37.7 (C-4), 38.4 (C-1), 40.4 (3-O-dimethylsuccinyl C-3'), 40.9 (C-8), 42.9 (C-14), 44.5 (28-O-dimethylglutaryl C-4", C-19), 44.7 (3-Odimethylsuccinyl C-2'), 45.1 (28-O-dimethylsuccinyl C-2"), 46.4 (C-17), 48.1 (C-18), 50.0 (C-9), 55.4 (C-5), 60.5 (AZT-C-3'), 62.8 (C-28, AZT-C-5'), 81.5 (C-3), 81.9 (AZT-C-4'), 85.3 (AZT-C-1'), 111.3 (AZT-C-5), 135.4 (AZT-C-6), 150.1 (AZT-C-2), 163.8 (AZT-C-4), 171.0 (3-O-dimethylsuccinyl 1'-COO-), 171.2 (28-O-dimethylglutaryl 5"-COO-AZT), 172.3 (28-O-dimethylglutaryl 1"-COO-), 181.8 (3-O-dimethylsuccinyl 4'-COOH). HRESIMS (positive) $\mathrm{m} / z 964.6037[\mathrm{M}+\mathrm{H}]^{+}\left(\right.$calcd for $\left.\mathrm{C}_{53} \mathrm{H}_{82} \mathrm{~N}_{5} \mathrm{O}_{11}, 964.6011\right)$.

\section{1-(3'-Azido-3'-deoxythymidine-5'-yl)-4-(3-O-glutaryl-lup-28-yl) 2,2-dimethylsuccinate}

(58): Yield $82.5 \%$ (starting from $20.4 \mathrm{mg}$ of 44 ); white solid. $[\alpha]_{\mathrm{D}}^{27}-1.9^{\circ}\left(c 1.11 \mathrm{CHCl}_{3}\right) .{ }^{1} \mathrm{H}$ $\mathrm{NMR}\left(\mathrm{CDCl}_{3}, 400 \mathrm{MHz}\right) \delta 0.77,0.84$ (each $\left.3 \mathrm{H}, \mathrm{d}, J=8.0 \mathrm{~Hz}, \mathrm{CH}_{3}-29,30\right), 0.85(6 \mathrm{H}, \mathrm{s}$, $\left.\mathrm{CH}_{3}-24, \mathrm{CH}_{3}-23\right), 0.87$ (3H, s, $\left.\mathrm{CH}_{3}-25\right), 0.95$ (3H, s, $\mathrm{CH}_{3}-27$ ), 1.02 (3H, s, $\left.\mathrm{CH}_{3}-26\right), 1.30$, 1.31 (each $3 \mathrm{H}, \mathrm{s}, 28-O$-dimethylsuccinyl $\mathrm{CH}_{3}$ ), $1.94(3 \mathrm{H}, \mathrm{br}$ s, AZT-CH3), 1.98 (2H, quint, $J=7.2 \mathrm{~Hz}, 3$ - $O$-glutaryl $\left.\mathrm{H}_{2}-3^{\prime}\right), 2.26-2.35,2.44-2.51$ (each $\left.1 \mathrm{H}, \mathrm{m}, \mathrm{AZT}-\mathrm{H}_{2}-2^{\prime}\right), 2.40(2 \mathrm{H}, \mathrm{t}$, $J=7.2 \mathrm{~Hz}, 3$ - $O$-glutaryl $\left.\mathrm{H}_{2}-2^{\prime}\right), 2.44\left(2 \mathrm{H}, \mathrm{t}, J=7.2 \mathrm{~Hz}, 3\right.$ - $O$-glutaryl $\left.\mathrm{H}_{2}-4^{\prime}\right), 2.60,2.74$ (each $1 \mathrm{H}, \mathrm{d}, J=16.0 \mathrm{~Hz}, 28$ - $O$-dimethylsuccinyl $\mathrm{H}_{2}-2 "$ ), 3.82, 4.28 (each $1 \mathrm{H}, \mathrm{d}, J=10.8 \mathrm{~Hz}$, $\left.\mathrm{H}_{2}-28\right), 4.09$ (1H, q-like, AZT-H-4'), 4.25-4.30 (1H, m, AZT-H-3'), $4.28(1 \mathrm{H}, \mathrm{dd}, J=4.0$, $12.0 \mathrm{~Hz}$, AZT-H-5a'), $4.51\left(1 \mathrm{H}, \mathrm{dd}, J=4.0,12.0 \mathrm{~Hz}, \mathrm{AZT}-\mathrm{H}_{2}-5 \mathrm{~b}^{\prime}\right), 4.49(1 \mathrm{H}, \mathrm{dd}, J=5.2$, $10.0 \mathrm{~Hz}, \mathrm{H}-3), 6.16(1 \mathrm{H}, \mathrm{t}-\mathrm{like}, J=6.4 \mathrm{~Hz}$, AZT-H-1'), $7.28(1 \mathrm{H}, \mathrm{d}, J=1.2 \mathrm{~Hz}$, AZT-H-6), $8.96(1 \mathrm{H}, \mathrm{br} \mathrm{s}, \mathrm{AZT}-\mathrm{NH}) ;{ }^{13} \mathrm{C} \mathrm{NMR}\left(\mathrm{CDCl}_{3}, 100 \mathrm{MHz}\right) \delta 12.4\left(\mathrm{AZT}-\mathrm{CH}_{3}\right), 14.6(\mathrm{C}-27)$, 14.9 (C-29), 16.0 (C-26), 16.1 (C-25), 16.6 (C-24), 18.2 (C-6), 20.1 (3-O-glutaryl C-3'), 20.8 (C-11), 21.6 (C-21), 22.9 (C-30), 23.7 (C-2), 25.1, 25.9 (28-O-dimethylsuccinyl $\mathrm{CH}_{3}$ ), 26.8 (C-12), 26.9 (C-15), 28.0 (C-23), 29.4 (C-20), 29.9 (C-16), 32.9 (3-O-glutaryl C-4'), 33.7 (3$O$-glutaryl C-2'), 34.2 (C-7), 34.7 (C-22), 37.1 (C-10), 37.2 (C-13), 37.5 (AZT-C-2'), 37.8 (C-4), 38.4 (C-1), 40.7 (28-O-dimethylsuccinyl C-3"), 40.9 (C-8), 42.9 (C-14), 44.6 (C-19, 28-O-dimethylsuccinyl C-2"), 46.5 (C-17), 48.2 (C-18), 50.0 (C-9), 55.4 (C-5), 60.4 (AZTC-3'), 63.2 (C-28), 63.3 (AZT-C-5'), 81.1 (C-3), 82.0 (AZT-C-4'), 84.9 (AZT-C-1'), 111.3 (AZT-C-5), 135.2 (AZT-C-6), 150.0 (AZT-C-2), 163.7 (AZT-C-4), 171.7 (28-Odimethylsuccinyl 1"-COO-), 172.7 (3-O-glutaryl 1'-COO-), 176.5 (28-O-dimethylsuccinyl 4"-COO-AZT), 177.1 (3-O-glutaryl 5'-COOH). HRESIMS (positive) $\mathrm{m} / \mathrm{z} 936.5716[\mathrm{M}+\mathrm{H}]^{+}$ (calcd for $\mathrm{C}_{51} \mathrm{H}_{78} \mathrm{~N}_{5} \mathrm{O}_{11}, 936.5698$ ).

4-(3'-Azido-3'-deoxythymidine-5'-yl)-1-(3-O-glutaryl-lup-28-yl) 2,2-dimethylsuccinate (59): Yield $66.1 \%$ (starting from $32.6 \mathrm{mg}$ of $\mathbf{4 5}$ ); white solid. $[\alpha]_{\mathrm{D}}^{27}+2.3^{\circ}\left(c 1.66 \mathrm{CHCl}_{3}\right) .{ }^{1} \mathrm{H}$ 
NMR $\left(\mathrm{CDCl}_{3}, 400 \mathrm{MHz}\right) \delta 0.77,0.84$ (each $\left.3 \mathrm{H}, \mathrm{d}, J=8.0 \mathrm{~Hz}, \mathrm{CH}_{3}-29,30\right), 0.85(6 \mathrm{H}, \mathrm{s}$, $\left.\mathrm{CH}_{3}-24, \mathrm{CH}_{3}-23\right), 0.87$ (3H, s, $\left.\mathrm{CH}_{3}-25\right), 0.96$ (3H, s, $\left.\mathrm{CH}_{3}-27\right), 1.04$ (3H, s, $\left.\mathrm{CH}_{3}-26\right), 1.31$, 1.34 (each $3 \mathrm{H}, \mathrm{s}, 28-O$-dimethylsuccinyl $\left.\mathrm{CH}_{3}\right), 1.96\left(3 \mathrm{H}\right.$, br s, AZT-CH $\left.{ }_{3}\right), 1.98(2 \mathrm{H}$, quint, $J$ $=7.2 \mathrm{~Hz}, 3-O$-glutaryl $\left.\mathrm{H}_{2}-3^{\prime}\right), 2.32-2.51\left(2 \mathrm{H}, \mathrm{m}, \mathrm{AZT}-\mathrm{H}_{2}-2^{\prime}\right), 2.40(2 \mathrm{H}, \mathrm{t}, J=7.2 \mathrm{~Hz}, 3-O-$ glutaryl $\left.\mathrm{H}_{2}-2^{\prime}\right), 2.43\left(2 \mathrm{H}, \mathrm{t}, J=7.2 \mathrm{~Hz}, 3-O\right.$-glutaryl $\left.\mathrm{H}_{2}-4^{\prime}\right), 2.61,2.66$ (each $1 \mathrm{H}, \mathrm{d}, J=16.4$ $\mathrm{Hz}, 28-O$-dimethylsuccinyl $\left.\mathrm{H}_{2}-2 "\right), 3.81,4.30$ (each $\left.1 \mathrm{H}, \mathrm{d}, J=10.8 \mathrm{~Hz}, \mathrm{H}_{2}-28\right), 4.05(1 \mathrm{H}, \mathrm{q}-$ like, AZT-H-4'), 4.17-4.23 (1H, m, AZT-H-3'), 4.26 (1H, dd, $J=3.6,12.4 \mathrm{~Hz}$, AZT-H-5a'), $4.42(1 \mathrm{H}, \mathrm{dd}, J=4.4,12.4 \mathrm{~Hz}$, AZT-H-5b'), $4.49(1 \mathrm{H}, \mathrm{dd}, J=5.2,10.8 \mathrm{~Hz}, \mathrm{H}-3), 6.14(1 \mathrm{H}$, t-like, $J=6.4 \mathrm{~Hz}$, AZT-H-1'), $7.31(1 \mathrm{H}, \mathrm{d}, J=0.8 \mathrm{~Hz}$, AZT-H-6), $9.14(1 \mathrm{H}$, br s, AZT$\mathrm{NH}) ;{ }^{13} \mathrm{C}$ NMR $\left(\mathrm{CDCl}_{3}, 100 \mathrm{MHz}\right) \delta 12.5\left(\mathrm{AZT}-\mathrm{CH}_{3}\right), 14.7(\mathrm{C}-27), 14.9(\mathrm{C}-29), 16.0$ (C-26), 16.1 (C-25), 16.6 (C-24), 18.2 (C-6), 20.1 (3-O-glutaryl C-3'), 20.8 (C-11), 21.6 (C-21), 22.9 (C-30), 23.7 (C-2), 25.5, 25.8 (28-O-dimethylsuccinyl $\left.\mathrm{CH}_{3}\right), 26.8$ (C-12), 26.9 (C-15), 28.0 (C-23), 29.4 (C-20), 29.9 (C-16), 32.9 (3-O-glutaryl C-4'), 33.7 (3-O-glutaryl C-2'), 34.2 (C-7), 34.7 (C-22), 37.0 (C-10), 37.2 (C-13), 37.5 (AZT-C-2'), 37.8 (C-4), 38.4 (C-1), 40.9 (C-8), 41.1 (28-O-dimethylsuccinyl C-2"), 42.9 (C-14), 43.9 (28-O-

dimethylsuccinyl C-3"), 44.6 (C-19), 46.7 (C-17), 48.2 (C-18), 50.0 (C-9), 55.4 (C-5), 60.3 (AZT-C-3'), 62.9 (AZT-C-5'), 63.3 (C-28), 81.1 (C-3), 81.8 (AZT-C-4'), 85.2 (AZT-C-1'), 111.3 (AZT-C-5), 135.5 (AZT-C-6), 150.1 (AZT-C-2), 163.8 (AZT-C-4), 170.8 (28-Odimethylsuccinyl 4"-COO-AZT), 172.7 (3-O-glutaryl 1'-COO-), 176.9 (28-Odimethylsuccinyl 1"-COO-), 177.3 (3-O-glutaryl 5'-COOH). HRESIMS (positive) $\mathrm{m} / \mathrm{z}$ $936.5694[\mathrm{M}+\mathrm{H}]^{+}$(calcd for $\left.\mathrm{C}_{51} \mathrm{H}_{78} \mathrm{~N}_{5} \mathrm{O}_{11}, 936.5698\right)$.

\section{3'-Azido-3'-deoxythymidine-5'-yl 3-O-glutaryl-lup-28-yl glutarate (60): Yield $587 \%$}

(starting from $41.2 \mathrm{mg}$ of 46); white solid. $[\alpha]_{\mathrm{D}}^{27}+7.0^{\circ}\left(c 1.40, \mathrm{CHCl}_{3}\right) .{ }^{1} \mathrm{H} \mathrm{NMR}\left(\mathrm{CDCl}_{3}\right.$, $400 \mathrm{MHz}$ ) $\delta 0.78,0.84$ (each $\left.3 \mathrm{H}, \mathrm{d}, J=8.0 \mathrm{~Hz}, \mathrm{CH}_{3}-29,30\right), 0.84\left(6 \mathrm{H}, \mathrm{s}, \mathrm{CH}_{3}-24, \mathrm{CH}_{3}-23\right.$ ), $0.87\left(3 \mathrm{H}, \mathrm{s}, \mathrm{CH}_{3}-25\right), 0.96\left(3 \mathrm{H}, \mathrm{s}, \mathrm{CH}_{3}-27\right), 1.05\left(3 \mathrm{H}, \mathrm{s}, \mathrm{CH}_{3}-26\right), 1.94(3 \mathrm{H}, \mathrm{d}, J=1.2 \mathrm{~Hz}$, AZT-CH 3 ) $1.97\left(2 \mathrm{H}\right.$, quint, $J=7.2 \mathrm{~Hz}, 3-O$-glutaryl $\left.\mathrm{H}_{2}-3^{\prime}\right), 1.99(2 \mathrm{H}$, quint, $J=7.2 \mathrm{~Hz}, 28$ $O$-glutaryl $\mathrm{H}_{2}-3$ "), 2.34-2.51 (2H, m, AZT- $\mathrm{H}_{2}-2$ '), $2.40(2 \mathrm{H}, \mathrm{t}, J=7.2 \mathrm{~Hz}, 3$ - $O$-glutaryl $\left.\mathrm{H}_{2}-2^{\prime}\right), 2.41\left(2 \mathrm{H}, \mathrm{t}, J=7.2 \mathrm{~Hz}, 28-O\right.$-glutaryl $\left.\mathrm{H}_{2}-2 "\right), 2.43(2 \mathrm{H}, \mathrm{t}, J=7.2 \mathrm{~Hz}, 3-O$-glutaryl $\left.\mathrm{H}_{2}-4^{\prime}\right), 2.46\left(2 \mathrm{H}, \mathrm{t}, J=7.2 \mathrm{~Hz}, 28\right.$ - $O$-glutaryl $\left.\mathrm{H}_{2}-4 "\right), 3.83,4.29$ (each $1 \mathrm{H}, \mathrm{d}, J=11.2 \mathrm{~Hz}$, $\mathrm{H}_{2}$-28), 4.07 (1H, q-like, AZT-H-4'), 4.20-4.24 (1H, m, AZT-H-3'), 4.32 (1H, dd, $J=4.0$, $12.0 \mathrm{~Hz}$, AZT-H-5a'), $4.40(1 \mathrm{H}, d d, J=4.4,12.0 \mathrm{~Hz}$, AZT-H-5b'), $4.49(1 \mathrm{H}, \mathrm{dd}, J=6.0$, $10.4 \mathrm{~Hz}, \mathrm{H}-3), 6.09$ (1H, t-like, $J=6.4 \mathrm{~Hz}$, AZT-H-1'), $7.23(1 \mathrm{H}$, br s, AZT-H-6), $9.10(1 \mathrm{H}$, br s, AZT-NH); ${ }^{13} \mathrm{C} \mathrm{NMR}\left(\mathrm{CDCl}_{3}, 100 \mathrm{MHz}\right) \delta 12.5$ (AZT-CH3), 14.6 (C-27), 14.9 (C-29), 16.0 (C-26, -25), 16.6 (C-24), 18.2 (C-6), 20.1 (28-O-glutaryl C-3", 3-O-glutaryl C-3'), 20.8 (C-11), 21.6 (C-21), 22.9 (C-30), 23.7 (C-2), 26.8 (C-12), 26.9 (C-15), 28.0 (C-23), 29.4 (C-20), 29.9 (C-16), 32.9 (3-O-glutaryl C-4'), 33.1 (28-O-glutaryl C-4"), 33.2 (28-O-glutaryl C-2"), 33.7 (3-O-glutaryl C-2'), 34.2 (C-7), 34.7 (C-22), 37.0 (C-10), 37.2 (C-13), 37.5 (AZT-C-2'), 37.8 (C-4), 38.4 (C-1), 41.0 (C-8), 42.9 (C-14), 44.6 (C-19), 46.6 (C-17), 48.2 (C-18), 50.0 (C-9), 55.4 (C-5), 60.6 (AZT-C-3'), 63.0 (C-28), 63.3 (AZT-C-5'), 81.1 (C-3), 81.8 (AZT-C-4'), 85.7 (AZT-C-1'), 111.3 (AZT-C-5), 135.5 (AZT-C-6), 150.0 (AZT-C-2), 163.7 (AZT-C-4), 172.3 (28- $O$-glutaryl 5"-COO-AZT), 172.7 (3- $O$-glutaryl 1'-COO-), 173.1 (28-O-glutaryl 1"-COO-), 177.2 (3-O-glutaryl 5'-COOH). HRESIMS (positive) $\mathrm{m} / z$ $922.5557[\mathrm{M}+\mathrm{H}]^{+}$(calcd for $\mathrm{C}_{60} \mathrm{H}_{76} \mathrm{~N}_{5} \mathrm{O}_{11}, 922.5541$ ).

\section{3'-Azido-3'-deoxythymidine-5'-yl 3-O-glutaryl-lup-28-yl 3,3-dimethylglutarate (61):}

Yield $75.6 \%$ (starting from $39 \mathrm{mg}$ of 47 ); white solid. $[\alpha]_{\mathrm{D}}^{27}+3.5^{\circ}(c 2.13, \mathrm{CHCl} 3) .{ }^{1} \mathrm{H}$ NMR $\left(\mathrm{CDCl}_{3}, 400 \mathrm{MHz}\right) \delta 0.77,0.84$ (each $\left.3 \mathrm{H}, \mathrm{d}, J=8.0 \mathrm{~Hz}, \mathrm{CH}_{3}-29,30\right), 0.85\left(6 \mathrm{H}, \mathrm{s}, \mathrm{CH}_{3}-24\right.$, $\left.\mathrm{CH}_{3}-23\right), 0.86$ (3H, s, $\left.\mathrm{CH}_{3}-25\right), 0.95$ (3H, s, $\left.\mathrm{CH}_{3}-27\right), 1.03$ (3H, s, $\left.\mathrm{CH}_{3}-26\right), 1.12,1.15$ (each $3 \mathrm{H}, \mathrm{s}, 28$ - $O$-dimethylglutaryl $\left.\mathrm{CH}_{3}\right), 1.95(3 \mathrm{H}, \mathrm{d}, J=1.2 \mathrm{~Hz}, \mathrm{AZT}-\mathrm{CH} 3), 1.97(2 \mathrm{H}$, quint, $J=$ $7.2 \mathrm{~Hz}, 3-O$-glutaryl $\left.\mathrm{H}_{2}-3^{\prime}\right), 2.32-2.51\left(2 \mathrm{H}, \mathrm{m}, \mathrm{AZT}-\mathrm{H}_{2}-2^{\prime}\right), 2.40(2 \mathrm{H}, \mathrm{t}, J=7.2 \mathrm{~Hz}, 3-O-$ glutaryl $\mathrm{H}_{2}-2^{\prime}$ ), 2.42, 2.49 (each $1 \mathrm{H}, \mathrm{d}, J=14.4 \mathrm{~Hz}, 28-O$-dimethylglutaryl $\mathrm{H}_{2}-2$ "), $2.43(2 \mathrm{H}$, 
t, $J=7.2 \mathrm{~Hz}, 3-O$-glutaryl $\left.\mathrm{H}_{2}-4^{\prime}\right), 2.48,2.56$ (each $1 \mathrm{H}, \mathrm{d}, J=15.2 \mathrm{~Hz}, 28-O-$

dimethylglutaryl $\mathrm{H}_{2}-4$ "), 3.79, 4.27 (each $1 \mathrm{H}, \mathrm{d}, J=10.8 \mathrm{~Hz}, \mathrm{H}_{2}-28$ ), 4.07 (1H, q-like, AZTH-4'), 4.20-4.28 (1H, m, AZT-H-3'), $4.26(1 \mathrm{H}, \mathrm{dd}, J=3.6,12.4 \mathrm{~Hz}$, AZT-H-5a'), $4.44(1 \mathrm{H}$, dd, $J=4.4,12.4 \mathrm{~Hz}$, AZT-H-5b'), 4.49 (1H, dd, $J=5.2,10.8 \mathrm{~Hz}, \mathrm{H}-3), 6.14$ (1H, t-like, $J=$ $6.4 \mathrm{~Hz}$, AZT-H-1'), $7.30\left(1 \mathrm{H}, \mathrm{d}, J=0.8 \mathrm{~Hz}\right.$, AZT-H-6), $9.18\left(1 \mathrm{H}, \mathrm{br}\right.$ s, AZT-NH); ${ }^{13} \mathrm{C}$ NMR $\left(\mathrm{CDCl}_{3}, 100 \mathrm{MHz}\right) \delta 12.5\left(\mathrm{AZT}-\mathrm{CH}_{3}\right), 14.6(\mathrm{C}-27), 14.9(\mathrm{C}-29), 16.0(\mathrm{C}-26,-25), 16.6$ (C-24), 18.2 (C-6), 20.1 (3-O-glutaryl C-3'), 20.8 (C-11), 21.6 (C-21), 22.9 (C-30), 23.7 (C-2), 26.8 (C-12), 26.9 (C-15), 27.9, 28.0 (28-O-dimethylglutaryl $\mathrm{CH}_{3}$ ), 28.0 (C-23), 29.4 (C-20), 29.9 (C-16), 32.6 (28-O-dimethylglutaryl C-3"), 32.9 (3-O-glutaryl C-4'), 33.7 (3-Oglutaryl C-2'), 34.2 (C-7), 34.7 (C-22), 37.0 (C-10), 37.2 (C-13), 37.6 (AZT-C-2'), 37.8 (C-4), 38.4 (C-1), 40.9 (C-8), 42.9 (C-14), 44.5 (28-O-dimethylglutaryl C-4" and C-19), 45.1 (28-O-dimethylglutaryl C-2"), 46.4 (C-17), 48.1 (C-18), 50.0 (C-9), 55.4 (C-5), 60.4 (AZT-C-3'), 62.7 (C-28 and AZT-C-5'), 81.1 (C-3), 81.9 (AZT-C-4'), 85.3 (AZT-C-1'), 111.3 (AZT-C-5), 135.4 (AZT-C-6), 150.1 (AZT-C-2), 163.8 (AZT-C-4), 171.2 (28-Odimethylglutaryl 5"-COO-AZT), 172.3 (28-O-dimethylglutaryl 1"-COO-), 172.7 (3-Oglutaryl 1'-COO-), 177.3 (3-O-glutaryl 5'-COOH). HRESIMS (positive) $\mathrm{m} / z$, 950.5876 [M $+\mathrm{H}]^{+}$(calcd for $\mathrm{C}_{52} \mathrm{H}_{80} \mathrm{~N}_{5} \mathrm{O}_{11}, 950.5854$ ).

\section{3'-Azido-3'-deoxythymidine-5'-yl 3-O-(3',3'-dimethylglutaryl)-lup-28-yl 3,3-}

dimethylglutarate (62): Yield $76.4 \%$ (starting from $87.7 \mathrm{mg}$ of $\mathbf{4 8}$ ); white solid. [ $\alpha]_{\mathrm{D}}^{29}$ $+0.64^{\circ}\left(c 2.78, \mathrm{CHCl}_{3}\right) .{ }^{1} \mathrm{H} \mathrm{NMR}\left(\mathrm{CDCl}_{3}, 400 \mathrm{MHz}\right) \delta 0.77,0.84$ (each $3 \mathrm{H}, \mathrm{d}, J=8.0 \mathrm{~Hz}$, $\left.\mathrm{CH}_{3}-29,30\right), 0.83\left(3 \mathrm{H}, \mathrm{s}, \mathrm{CH}_{3}-24\right), 0.85\left(6 \mathrm{H}, \mathrm{s}, \mathrm{CH}_{3}-23, \mathrm{CH}_{3}-25\right), 0.95\left(3 \mathrm{H}, \mathrm{s}, \mathrm{CH}_{3}-27\right)$, $1.03(3 \mathrm{H}, \mathrm{s}, \mathrm{CH} 3-26), 1.15\left(6 \mathrm{H}, \mathrm{s}, 3-O\right.$-dimethylglutaryl $\left.\mathrm{CH}_{3}\right), 1.12,1.15$ (each $3 \mathrm{H}, \mathrm{s}, 28$ $O$-dimethylglutaryl $\left.\mathrm{CH}_{3}\right), 1.95\left(3 \mathrm{H}\right.$, br s, AZT- $\left.\mathrm{CH}_{3}\right), 2.32-2.51\left(2 \mathrm{H}, \mathrm{m}, \mathrm{AZT}-\mathrm{H}_{2}-2^{\prime}\right), 2.41$, 2.47 (each $1 \mathrm{H}, \mathrm{d}, J=14.4 \mathrm{~Hz}, 3$ - $O$-dimethylglutaryl $\mathrm{H}_{2}-2$ '), 2.42, 2.48 (each $1 \mathrm{H}, \mathrm{d}, J=14.4$ $\mathrm{Hz}, 28$ - $O$-dimethylglutaryl $\left.\mathrm{H}_{2}-2 "\right), 2.47$ (2H, s, 3-O-dimethylglutaryl $\left.\mathrm{H}_{2}-4^{\prime}\right), 2.48,2.56$ (each $1 \mathrm{H}, \mathrm{d}, J=15.2 \mathrm{~Hz}, 28$ - $O$-dimethylglutaryl $\mathrm{H}_{2}-4 "$ ), $3.79,4.27$ (each $1 \mathrm{H}, \mathrm{d}, J=10.8 \mathrm{~Hz}$, $\left.\mathrm{H}_{2}-28\right), 4.07$ (1H, q-like, AZT-H-4'), 4.20-4.28 (1H, m, AZT-H-3'), 4.26 (1H, dd, $J=3.6$, $12.0 \mathrm{~Hz}$, AZT-H-5a'), 4.44 (1H, dd, $J=4.4,12.0 \mathrm{~Hz}$, AZT-H-5b'), $4.50(1 \mathrm{H}, \mathrm{dd}, J=5.2$, $10.8 \mathrm{~Hz}, \mathrm{H}-3), 6.14$ (1H, t-like, $J=6.4 \mathrm{~Hz}$, AZT-H-1'), 7.29 (1H, d, $J=1.2 \mathrm{~Hz}$, AZT-H-6), $9.19\left(1 \mathrm{H}\right.$, br s, AZT-NH); ${ }^{13} \mathrm{C} \mathrm{NMR}\left(\mathrm{CDCl}_{3}, 100 \mathrm{MHz}\right) \delta 12.5\left(\mathrm{AZT}^{-\mathrm{CH}_{3}}\right), 14.6(\mathrm{C}-27)$, 14.9 (C-29), 16.0 (C-26, -25), 16.6 (C-24), 18.2 (C-6), 20.8 (C-11), 21.6 (C-21), 22.9 (C-30), 23.8 (C-2), 26.8 (C-12), 26.9 (C-15), 27.9 (28-O-dimethylglutaryl $\left.\mathrm{CH}_{3}\right), 27.9,28.0$ (3-O-dimethylglutaryl $\left.\mathrm{CH}_{3}\right), 28.0$ (C-23), 29.4 (C-20), 29.9 (C-16), 32.6 (28-Odimethylglutaryl C-3"), 32.7 (3-O-dimethylglutaryl C-3'), 34.2 (C-7), 34.7 (C-22), 37.0 (C-10), 37.2 (C-13), 37.6 (AZT-C-2'), 37.7 (C-4), 38.4 (C-1), 40.9 (C-8), 42.9 (C-14), 44.6 (28-O-dimethylglutaryl C-4" and C-19), 45.1 (28-O-dimethylglutaryl C-2"), 45.2 (3-O glutaryl C-4'), 45.7 (3-O-glutaryl C-2'), 46.4 (C-17), 48.1 (C-18), 50.0 (C-9), 55.4 (C-5), 60.4 (AZT-C-3'), 62.7 (C-28, and AZT-C-5'), 81.4 (C-3), 81.8 (AZT-C-4'), 85.3 (AZT-C-1'), 111.3 (AZT-C-5), 135.4 (AZT-C-6), 150.1 (AZT-C-2), 163.8 (AZT-C-4), 171.2 (28-Odimethylglutaryl 5"-COO-AZT), 172.3 (28-O-dimethylglutaryl 1"-COO-, 3-Odimethylglutaryl 1'-COO-), 175.6 (3-O-dimethylglutaryl 5'-COOH). HRESIMS (positive) $m / z, 1000.5991[\mathrm{M}+\mathrm{Na}]^{+}$(calcd for $\mathrm{C}_{54} \mathrm{H}_{8} 3 \mathrm{~N}_{5} \mathrm{O}_{11} \mathrm{Na}, 1000.5987$ ).

HIV-1 NL4-3 Inhibition Assay in MT-4 Lymphocytes: A previously described procedure was used in the experiments. ${ }^{21,23}$

\section{Supplementary Material}

Refer to Web version on PubMed Central for supplementary material. 


\section{Acknowledgments}

This investigation was supported by Grant-in-Aid for Scientific Research (C) from the Japan Society for the Promotion of Science (Y.K.) as well as a Grant AI-077417 from the National Institute of Allergy and Infectious Diseases (K.H.L.).

\section{References and notes}

1. Cichewicz RH, Kouzi SA. Med. Res. Rev 2004;24:90. [PubMed: 14595673]

2. Alakurtti S, Mäkelä T, Koskimies S, Yli-Kauhaluoma J. Eur. J. Pharm. Sci 2006;29:1. [PubMed: 16716572]

3. Yu D, Morris-Natschke SL, Lee KH. Med. Res. Rev 2007;27:108. [PubMed: 16888749]

4. Gautam R, Jachak SM. Med. Res. Rev 2009;29:767. [PubMed: 19378317]

5. Mullauer FB, Kessler JH, Medema JP. Anticancer Drugs 2010;21:215. [PubMed: 20075711]

6. Kashiwada Y, Hashimoto F, Cosentino LM, Chen CH, Garrett PE, Lee KH. J. Med. Chem 1996;39:1016. [PubMed: 8676334]

7. Martin DE, Blum R, Wilton J, Doto J, Galbraith H, Burgess GL, Smith PC, Ballow C. Antimicrob. Agents Chemother 2007;51:3063. [PubMed: 17576843]

8. Smith PC, Ogundele A, Forrest A, Wilton J, Salzwedel K, Doto J, Allaway PA, Martin DE. Antimicrob. Agents Chemother 2007;51:3574. [PubMed: 17638699]

9. http://www.myriadpharma.com/product-pipeline/clinical/mpc-4326\#4326-recent-presentations.

10. Li F, Goila-Gaur R, Salzwedel K, Kilgore NR, Reddick M, Matallana C, Castillo A, Zoumplis D, Marin DE, Orenstein JM, Allaway GP, Freed EO, Wild CT. Proc. Natl. Acad. Sci. USA 2003;100:13555. [PubMed: 14573704]

11. Chen IC, Shen JK, Wang HK, Cosentino LM, Lee KH. Bioorg. Med. Chem. Lett 1998;8:1267. [PubMed: 9871747]

12. Chen IC, Wang HK, Kashiwada Y, Shen JK, Cosentino LM, Chen CH, Yang LM, Lee KH. J. Med. Chem 1998;41:4648. [PubMed: 9804704]

13. Kashiwada Y, Chiyo J, Ikeshiro Y, Nagao T, Okabe H, Cosentino LM, Fowke K, Lee KH. Bioorg. Med. Chem. Lett 2001;11:183. [PubMed: 11206454]

14. Kashiwada Y, Sekiya M, Ikeshiro Y, Fujioka T, Kilgore NR, Wild CT, Allaway GP, Lee KH. Bioorg. Med. Chem. Lett 2004;14:5851. [PubMed: 15501054]

15. Anti-HIV data for compounds $\mathbf{2 , 4 , 5}$, and $\mathbf{6}$ evaluated by the HIV-1IIIB infected H9 lymphocytes assay, 2: $\mathrm{EC}_{50}<0.00035 \mu \mathrm{M}$, TI $>20,000,4: \mathrm{EC}_{50} 2 \times 10^{-5} \mu \mathrm{M}$, TI $1.12 \times 10^{6}, 5: \mathrm{EC}_{50} 0.00066$ $\mu \mathrm{M}$, TI 21,515, 6: $\mathrm{EC}_{50} 0.00087 \mu \mathrm{M}$, TI 42,400.

16. Zimmermann GR, Lehra J, Keith CT. Drug Discov. Today 2007;12:34. [PubMed: 17198971]

17. Jia J, Zhu F, Ma XH, Cao ZW, Li YX, Chen YZ. Nat. Rev. Drug Discov 2009;8:111. [PubMed: 19180105]

18. Morphy R, Kay C, Rankovic Z. Drug Discov. Today 2004;9:641. [PubMed: 15279847]

19. Mitsuya H, Weinhold KJ, Furman PA, St. Clair MH, Nusinoff Lehrman S, Gallo RC, Bolognesi D, Barry DW, Broder S. Proc. Natl. Acad. Sci. USA 1985;82:7096. [PubMed: 2413459]

20. Hashimoto F, Kashiwada Y, Cosentino LM, Chen CH, Garrett PE, Lee KH. Bioorg. Med. Chem 1997;5:2133. [PubMed: 9459011]

21. Qian KD, Yu DL, Chen CH, Huang L, Morris-Natschke SL, Nitz TJ, Salzwedel K, Reddick M, Allaway GP, Lee KH. J. Med. Chem 2009;52:3248. [PubMed: 19388685]

22. Qian KD, Kuo RY, Chen CH, Huang L, Morris-Natschke SL, Lee KH. J. Med. Chem 2010;53:3133. [PubMed: 20329730]

23. Yu DL, Sakurai Y, Chen CH, Chang FR, Huang L, Kashiwada Y, Lee KH. J. Med. Chem 2006;49:5462. [PubMed: 16942019] 


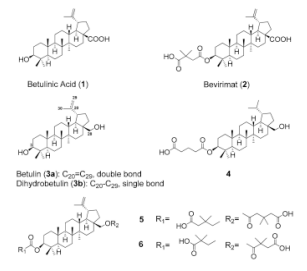

Figure1.

Betulinic acid, betulin and their derivatives which demonstrated potent anti-HIV activities. 


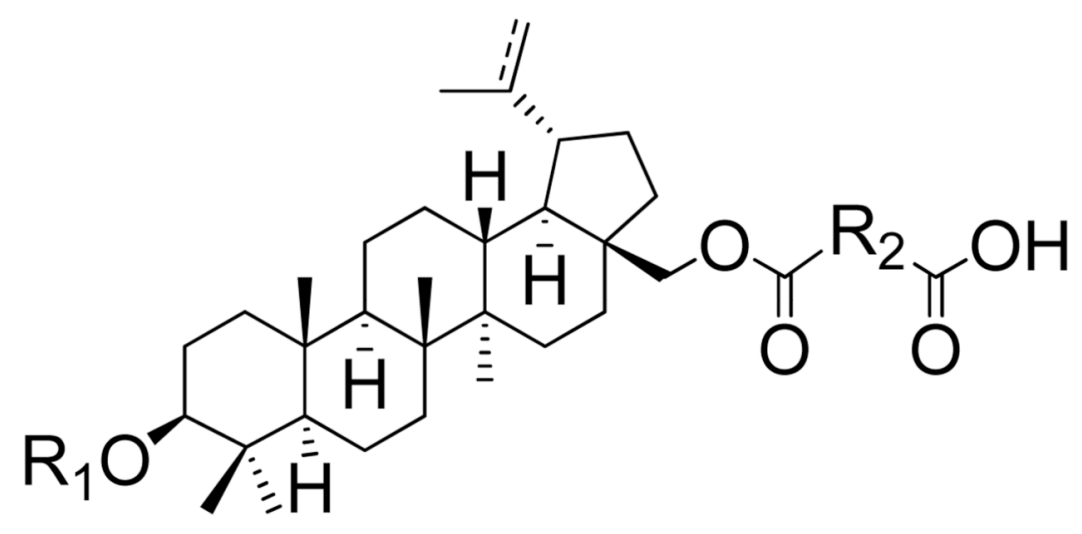<smiles></smiles>

3,28-di-O-acylbetulins

AZT

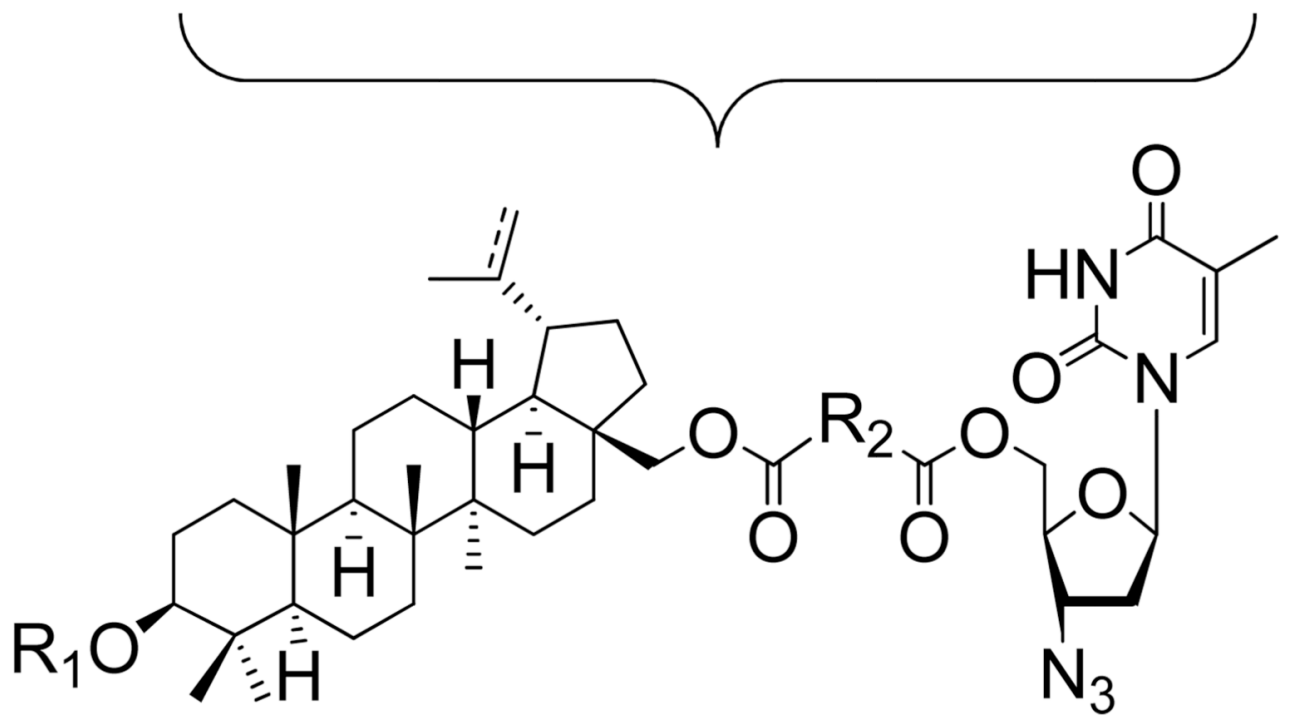

Figure 2.

General structure of hybrid-type anti-HIV agents. 


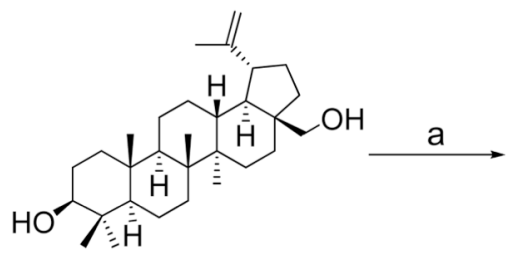

Betulin (3a)

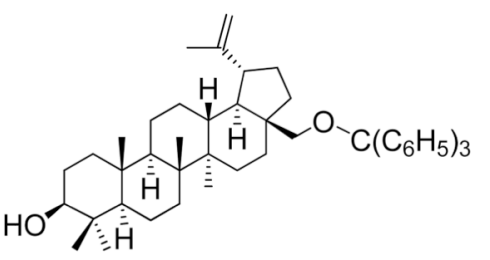

7
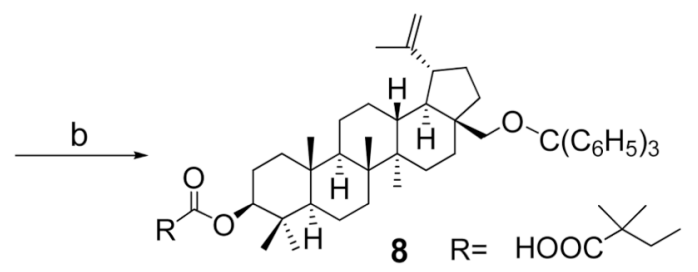

$9 \mathrm{R}=\mathrm{HOOC} \chi$

$10 \mathrm{R}=\mathrm{HOOC}$

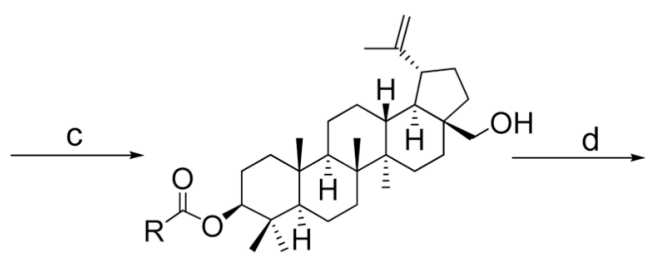

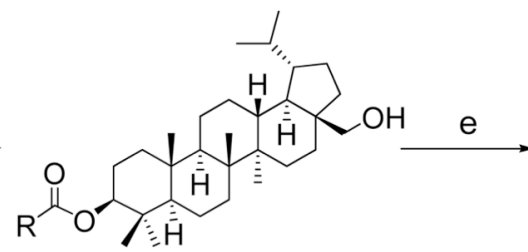

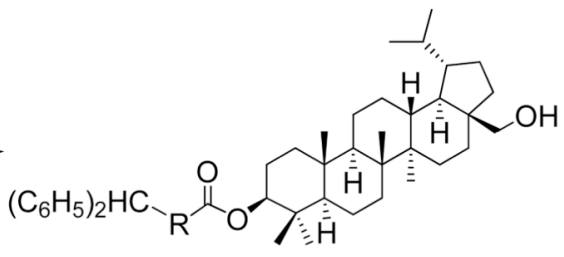

11<smiles>[R]OOC(=O)C(C)(C)CC</smiles>

12<smiles>[R]OOCC(C)(C)CC</smiles>

13<smiles></smiles>

$4 \mathrm{R}=\mathrm{HOOC}$<smiles></smiles>

15<smiles>[R]OOC(=O)CC(C)(C)CC</smiles>

$18 \mathrm{R}=-\frac{1}{1}$

19<smiles>[R]OC(=O)CC(C)(C)CC</smiles>

20<smiles>[R]COC(=O)CCCC</smiles>

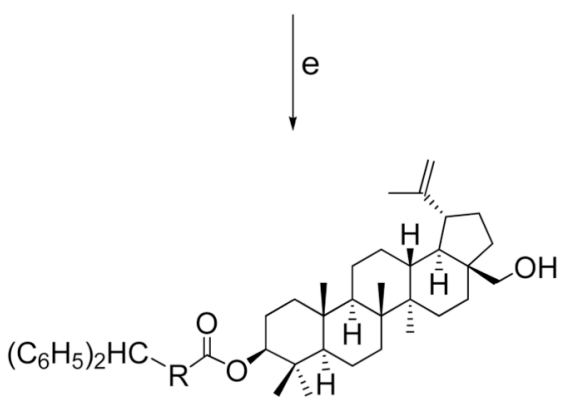

16<smiles>[R]COC(=O)C(C)(C)CC</smiles>

17<smiles>[R]COC(=O)CC(C)(C)CC</smiles>

Scheme 1.

Reagents and conditions: (a) trityl chloride, DAMP, DMF, reflux; (b) dicarboxylic acid (or anhydride), DMAP, pyridine, reflux; (c) PPTS, $\mathrm{CH}_{2} \mathrm{Cl}_{2}-\mathrm{EtOH}$ or $\mathrm{FeCl}_{3}, 95 \% \mathrm{THF}$, reflux; (d) $\mathrm{H}_{2}, 10 \% \mathrm{Pd} / \mathrm{C}$, EtOAc-EtOH, r.t.; (e) diphenyldiazomethane, $\mathrm{MeOH}$, r.t. 


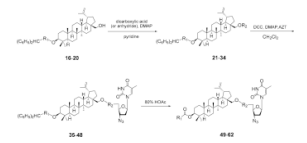

Scheme 2.

General synthetic route to conjugates of betulin derivatives with AZT. 


\section{Table 1}

Ester Side Chains of 21-62.

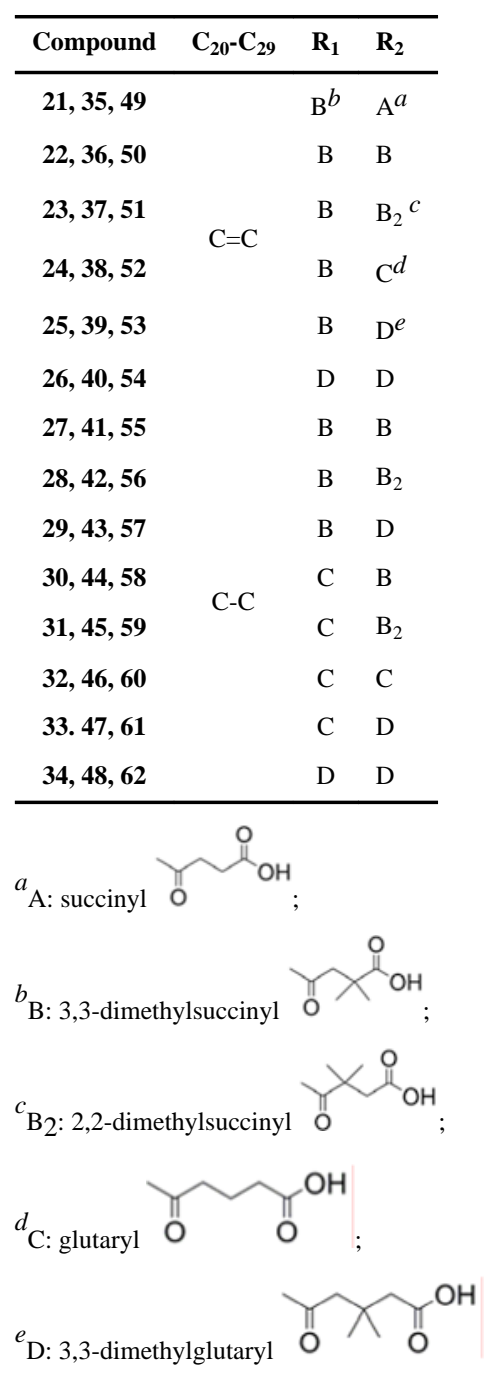


Table 2

Anti-HIV-1 Data of 49-62 Against HIV-1 ${ }_{\text {NL4-3 Infected MT-4 Cells }}{ }^{a}$

\begin{tabular}{|c|c|c|c|}
\hline Compound & $\mathrm{EC}_{50} b(\mu \mathrm{M})$ & $\mathrm{IC}_{50} c(\mu \mathrm{M})$ & $\mathrm{TI}^{d}$ \\
\hline 49 & 0.045 & 7.1 & 158 \\
\hline 50 & 0.098 & 7.0 & 71 \\
\hline 51 & 0.040 & 5.4 & 135 \\
\hline 52 & 0.060 & 8.7 & 145 \\
\hline 53 & 0.063 & 8.1 & 129 \\
\hline 54 & 0.18 & 7.7 & 43 \\
\hline 55 & 0.087 & 9.0 & 103 \\
\hline 56 & 0.056 & 7.7 & 138 \\
\hline 57 & 0.29 & 9.0 & 31 \\
\hline 58 & 0.26 & 7.2 & 28 \\
\hline 59 & 0.073 & 8.0 & 110 \\
\hline 60 & 0.093 & 9.0 & 97 \\
\hline 61 & 1.21 & 8.5 & 7 \\
\hline 62 & 0.36 & 9.8 & 27 \\
\hline 2 & $0.096^{e}$ & $>5^{e}$ & $>52^{e}$ \\
\hline $\mathrm{AZT}$ & 0.027 & $>37.4$ & $>1385$ \\
\hline
\end{tabular}

${ }^{a}$ Data presented are averages of at least two separate experiments;

${ }^{b}$ Concentration that inhibits HIV-1NL4-3 replication by $50 \%$;

${ }^{c}$ Concentration that inhibits mock-infected MT- 4 cell growth by $50 \%$;

$d_{\mathrm{TI}}=\mathrm{IC} 50 / \mathrm{EC} 50 ;$

${ }^{e}$ Data are taken from reference 23 
Table 3

Anti-HIV-1 Data of Conjugates 49, 52 and Analogous Carboxylic Acids 63, 64 Against HIV-1 NL4-3 Infected MT-4 Cells ${ }^{a}$

\begin{tabular}{|c|c|c|c|}
\hline O & & $\begin{array}{l}49, n=2, \\
52, n=3 \\
63, n=2, \\
64, n=3\end{array}$ & \\
\hline Compound & $\mathrm{EC}_{50}{ }^{b}(\mu \mathrm{M})$ & $\mathrm{IC}_{50}{ }^{c}(\mu \mathrm{M})$ & $\mathrm{TI}^{d}$ \\
\hline 49 & 0.045 & 7.1 & 158 \\
\hline 63 & 0.18 & 11.3 & 63 \\
\hline 52 & 0.060 & 8.7 & 145 \\
\hline 64 & 0.310 & $>14.6$ & $>47$ \\
\hline 2 & $0.096^{e}$ & $>5^{e}$ & $>52^{e}$ \\
\hline AZT & 0.027 & $>37.4$ & $>1385$ \\
\hline
\end{tabular}

${ }^{a}$ Data presented are averages of at least two separate experiments;

${ }^{b}$ Concentration that inhibits HIV-1NL4-3 replication by $50 \%$;

${ }^{c}$ Concentration that inhibits mock-infected MT-4 cell growth by 50\%;

$d_{\mathrm{TI}}=\mathrm{IC}_{50} / \mathrm{EC}_{50}$;

${ }^{e}$ Data are taken from reference ${ }^{23}$. 